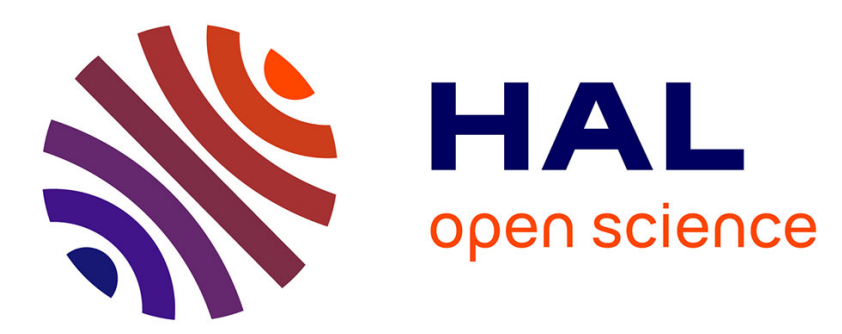

\title{
Characterisation of sets of limit measures of a cellular automaton iterated on a random configuration
}

Benjamin Hellouin de Menibus, Mathieu Sablik

\section{To cite this version:}

Benjamin Hellouin de Menibus, Mathieu Sablik. Characterisation of sets of limit measures of a cellular automaton iterated on a random configuration. Ergodic Theory and Dynamical Systems, 2016, 10.1017/etds.2016.46 . hal-01299001

\section{HAL Id: hal-01299001 https://hal.science/hal-01299001}

Submitted on 7 Apr 2016

HAL is a multi-disciplinary open access archive for the deposit and dissemination of scientific research documents, whether they are published or not. The documents may come from teaching and research institutions in France or abroad, or from public or private research centers.
L'archive ouverte pluridisciplinaire HAL, est destinée au dépôt et à la diffusion de documents scientifiques de niveau recherche, publiés ou non, émanant des établissements d'enseignement et de recherche français ou étrangers, des laboratoires publics ou privés. 


\title{
CHARACTERISATION OF SETS OF LIMIT MEASURES OF A CELLULAR AUTOMATON ITERATED ON A RANDOM CONFIGURATION
}

\author{
BENJAMIN HELLOUIN DE MENIBUS AND MATHIEU SABLIK
}

\begin{abstract}
The asymptotic behaviour of a cellular automaton iterated on a random configuration is well described by its limit probability measure(s). In this paper, we characterise measures and sets of measures that can be reached as limit points after iterating a cellular automaton on a simple initial measure. In addition to classical topological constraints, we exhibit necessary computational obstructions. With an additional hypothesis of connectivity, we show these computability conditions are sufficient by constructing a cellular automaton realising these sets, using auxiliary states in order to perform computations. Adapting this construction, we obtain a similar characterisation for the Cesàro mean convergence, a Rice theorem on the sets of limit points, and we are able to perform computation on the set of measures, i.e. the cellular automaton converges towards a set of limit points that depends on the initial measure. Last, under non-surjective hypotheses, it is possible to remove auxiliary states from the construction.
\end{abstract}

\section{INTRODUCTION}

A cellular automaton is a complex system defined by a local rule which acts synchronously and uniformly on the configuration space $\mathcal{A}^{\mathbb{Z}}$, where $\mathcal{A}$ is a finite alphabet. These simple models have a wide variety of different dynamical behaviours. We are interested in the typical asymptotic behaviour starting from a random configuration, as this is usually done in simulations; different approaches stemmed from such observations. It is well-described by taking the iterated image of the initial measure under the action of the cellular automaton, and considering the limit points of this sequence in the weak* topology.

It is natural to ask which sets of measures can be obtained as limit points in this way. Obviously, any measure can be reached by iterating the identity on itself. Therefore, a more interesting approach is to start from some simple measure such as the uniform Bernoulli measure. In some sense, this is similar to SRB measures which are "physically" relevant invariant measures obtained when starting from the Lebesgue measure in continuous dynamical systems [You02].

Formally speaking, given a simple initial measure $\mu$, we want to characterise all reachable $\mathcal{V}(F, \mu)$, the sets of accumulation points of the sequence $\left(F_{*}^{t} \mu\right)_{t \in \mathbb{N}}$ of the images of $\mu$ under the iterated action

of $F$, and $\mathcal{V}^{\prime}(F, \mu)$, the sets of accumulation points of $\left(\frac{1}{t+1} \sum_{i=0}^{t} F_{*}^{i} \mu\right)_{t \in \mathbb{N}}$, the Cesàro mean of the previous sequence, for all possible cellular automata $F$.

Previous works focused on the $\mu$-limit set, which corresponds to the union of the support of the limit measures [KM00, Ku05]. Very complex $\mu$-limit sets can be constructed [BPT06, BDS10], and our construction is partly inspired from these works.

Describing limit measures has been done for only few concrete nontrivial examples. There are essentially two types of convergence quite well understood:

2010 Mathematics Subject Classification. 37B10,37B15,03D10,03D80.

Key words and phrases. Symbolic Dynamics, Cellular automata, SRB measures,Turing machines. 
- convergence towards a simple measure: for example, the cyclic cellular automaton on three states introduced in [Fis90], starting from a Bernoulli measure, converges towards a linear combination of Dirac measures supported by uniform configurations dMS11;

- randomisation phenomenon for linear cellular automata: the Cesàro mean sequence of the iteration of a linear cellular automaton on a initial measure converges to the uniform Bernoulli measure as soon as the initial measure is in a large class which contains Markov measures [Lin84, FMMN00, MM98, PY02].

For any cellular automaton, starting from a Bernoulli measure or a Markov measure, we obtain after a finite number of steps a hidden Markov chain which is well understood [BP11]. If we consider a computable initial measure $\mu$ (which means that there is an algorithm that approximates at a known rate the probability that a word $u \in \mathcal{A}^{*}$ appears), then it is easy to see that $F_{*}^{t} \mu$ is also computable. For example, a Bernoulli or Markov measure is computable iff its parameters are computable real numbers.

The limit measure(s) are not necessarily computable since the speed of convergence is not known. Nevertheless, we show in Section 2 that there exists necessary computational obstructions. The main problem is to prove the reciprocal, in other words: given a set of measures satisfying the computational obstructions, construct a cellular automaton which, starting on any simple initial measure, reaches exactly this set asymptotically. Similar computational obstructions appear when characterising possible topological dynamics properties of subshifts of finite type or cellular automata: possible entropies [HM10], possible growth-type invariants [Mey11, possible sub-actions [Hoc09, AS11]... However, the construction is quite different here since starting from a random configuration requires to self-organise the space, in the same spirit as the probabilistic cellular automaton of [Gác01] which corrects the random perturbations.

In Section 3, we construct a cellular automaton $F$ such that, starting from any shift-mixing probability measure $\mu$ with full support, the limit points of the sequence of measures $\left(F_{*}^{t} \mu\right)_{t \in \mathbb{N}}$ are described as the accumulation points of a computable polygonal path of measures supported by periodic orbits. First of all the cellular automaton divides the initial configuration in segments and formats each segment using a method similar to the one developed in [DPST11. Computation takes place in a negligible part of each segment and the result is copied periodically on the rest of the segment. In order to have an arbitrarily large area of computation, segments are merged progressively in a controlled manner. The difficulty of the construction is to synchronise all the operations to ensure the convergence.

In the Section 4, we modify this construction so that we do not need auxiliary states, i.e., the cellular automaton only uses the same alphabet as the limit measure(s). This is only possible, however, at the price of some additional hypotheses on the support of the measures.

In Section 5 we use these constructions to answer various questions, along with some open questions. The results are, for a fixed measure $\mu$ in a large class of measures:

- characterisation of shift-invariant measures $\nu$ such that there exists a cellular automaton $F$ which verifies $F_{*}^{t} \mu \underset{t \rightarrow \infty}{\longrightarrow} \nu$ (Corollary 1);

- characterisation of connected subsets of shift-invariant measures $\mathcal{K}$ such that there exists a cellular automaton $F$ which verifies $\mathcal{V}(F, \mu)=\mathcal{K}$ (Corollary 2);

- characterisation of subsets of shift-invariant measures $\mathcal{K}^{\prime}$ such that there exists a cellular automaton $F$ which verifies $\mathcal{V}^{\prime}(F, \mu)=\mathcal{K}^{\prime}$ (Corollary 4);

- Rice theorem for shift-invariant measures and connected subsets of shift-invariant measures reached by a cellular automaton (Corollary 7 ).

- counterparts of all these results without auxiliary states, using the modified construction (Corollaries 3, 6] and 8). 
In Section 5.4, we consider the case where the set of limit points depends on the initial measure. Computational constraints appear to describe functions $\mu \longmapsto \mathcal{V}(F, \mu)$ that can be realised in this way. Indeed, it is possible to "transfer" the computational complexity of the initial measure (using it as an oracle) to the set of limit points. Modifying the construction of Section 3, we manage to build a set of limit points depending on the density of a special state; however, we do not obtain a complete characterisation.

\section{Definitions}

\subsection{Configuration space and cellular automata}

Let $\mathcal{A}$ be a finite alphabet. Consider $\mathcal{A}^{\mathbb{Z}}$ the space of configurations which are $\mathbb{Z}$-indexed sequences in $\mathcal{A}$. If $\mathcal{A}$ is endowed with the discrete topology, $\mathcal{A}^{\mathbb{Z}}$ is compact, perfect and totally disconnected in the product topology. Moreover one can define a metric on $\mathcal{A}^{\mathbb{Z}}$ compatible with this topology:

$$
\forall x, y \in \mathcal{A}^{\mathbb{Z}}, \quad d_{C}(x, y)=2^{-\min \left\{|i|: x_{i} \neq y_{i}, i \in \mathbb{Z}\right\}} .
$$

Let $\mathbb{U} \subset \mathbb{Z}$. For $x \in \mathcal{A}^{\mathbb{Z}}$, denote $x_{\mathbb{U}} \in \mathcal{A}^{\mathbb{U}}$ the restriction of $x$ to $\mathbb{U}$. Given a pattern $w \in \mathcal{A}^{\mathbb{U}}$, one defines the cylinder $[w]_{\mathbb{U}}=\left\{x \in \mathcal{A}^{\mathbb{Z}}: x_{\mathbb{U}}=w\right\}$. Denote $\mathcal{A}^{*}=\bigcup_{n} \mathcal{A}^{n}$ the set of all finite words $w=w_{0} \ldots w_{n-1} ;|w|=n$ is the length of $w$. Also denote $[w]_{i}=[w]_{[i, i+|w|-1]}$ and $[w]=[w]_{0}=$ $[w]_{[0,|w|-1]}$, and $\mathcal{A}^{\leq k}=\bigcup_{n \leq k} \mathcal{A}^{n}$. For any $u \in \mathcal{A}^{*}$ such that $|u| \leq|w|$, define the frequency of $u$ in $w$ as $\operatorname{Freq}(u, w)=\frac{1}{|w|-|u|+1} \operatorname{Card}\left(\left\{i \in[0,|w|-|u|]: w_{[i, i+|u|]}=u\right\}\right)$.

The shift map $\sigma: \mathcal{A}^{\mathbb{Z}} \rightarrow \mathcal{A}^{\mathbb{Z}}$ is defined by $\sigma(x)_{i}=x_{i+1}$ for $x=\left(x_{m}\right)_{m \in \mathbb{Z}} \in \mathcal{A}^{\mathbb{Z}}$ and $i \in \mathbb{Z}$ and a subshift is a closed $\sigma$-invariant subset of $\mathcal{A}^{\mathbb{Z}}$. For $w \in \mathcal{A}^{*},{ }^{\infty} w^{\infty}$ is the $\sigma$-periodic word defined by ${ }^{\infty} w_{[0,|w|-1]}^{\infty}=w$ and $\sigma^{i+|w|}\left({ }^{\infty} w^{\infty}\right)=\sigma^{i}\left({ }^{\infty} w^{\infty}\right)$ for all $i \in \mathbb{Z}$.

A cellular automaton $(\mathrm{CA})$ is a pair $\left(\mathcal{A}^{\mathbb{Z}}, F\right)$ where $F: \mathcal{A}^{\mathbb{Z}} \rightarrow \mathcal{A}^{\mathbb{Z}}$ is a continuous function that commutes with the shift $(\sigma \circ F=F \circ \sigma)$. By the Curtis-Hedlund-Lyndon theorem [Hed69], it is equivalent to a function defined by $F(x)_{i}=\bar{F}\left(\left(x_{i+u}\right)_{u \in \mathbb{U}_{F}}\right)$ for all $x \in \mathcal{A}^{\mathbb{Z}}$ and $i \in \mathbb{Z}$, where $\mathbb{U}_{F} \subset \mathbb{Z}$ is a finite set named neighbourhood and $\bar{F}: \mathcal{A}^{\mathbb{U}_{F}} \rightarrow \mathcal{A}$ is a local rule.

\subsection{Sets of measures on $\mathcal{A}^{\mathbb{Z}}$}

\subsubsection{Dynamical properties}

Let $\mathfrak{B}$ be the Borel sigma-algebra of $\mathcal{A}^{\mathbb{Z}}$. Denote by $\mathcal{M}\left(\mathcal{A}^{\mathbb{Z}}\right)$ the set of probability measures on $\mathcal{A}^{\mathbb{Z}}$ defined on the sigma-algebra $\mathfrak{B}$. Let $\mathcal{M}_{\sigma}\left(\mathcal{A}^{\mathbb{Z}}\right)$ be the $\sigma$-invariant probability measures on $\mathcal{A}^{\mathbb{Z}}$, that is to say the measures $\mu$ such that $\mu\left(\sigma^{-1}(B)\right)=\mu(B)$ for all $B \in \mathfrak{B}$. Cylinders corresponding to finite words form a base of the topology, so a measure $\mu \in \mathcal{M}_{\sigma}\left(\mathcal{A}^{\mathbb{Z}}\right)$ is entirely characterised by $\left\{\mu([u]): u \in \mathcal{A}^{*}\right\}$.

Usually $\mathcal{M}_{\sigma}\left(\mathcal{A}^{\mathbb{Z}}\right)$ is endowed with the weak* topology: a sequence $\left(\mu_{n}\right)_{n \in \mathbb{N}}$ in $\mathcal{M}_{\sigma}\left(\mathcal{A}^{\mathbb{Z}}\right)$ converges to $\mu \in \mathcal{M}_{\sigma}\left(\mathcal{A}^{\mathbb{Z}}\right)$ if and only if, for all finite subsets $\mathbb{U} \subset \mathbb{Z}$ and for all patterns $u \in \mathcal{A}^{\mathbb{U}}$, one has $\lim _{n \rightarrow \infty} \mu_{n}\left([u]_{\mathbb{U}}\right)=\mu\left([u]_{\mathbb{U}}\right)$. In the weak* topology, the set $\mathcal{M}_{\sigma}\left(\mathcal{A}^{\mathbb{Z}}\right)$ is compact and metrisable. A metric is defined by

$$
d_{\mathcal{M}}(\mu, \nu)=\sum_{n \in \mathbb{N}} \frac{1}{2^{n}} \max _{u \in \mathcal{A}^{n}}|\mu([u])-\nu([u])| .
$$

Define the ball centered on $\mu \in \mathcal{M}_{\sigma}\left(\mathcal{A}^{\mathbb{Z}}\right)$ of radius $\varepsilon>0$ as

$$
\mathbf{B}(\mu, \varepsilon)=\left\{\nu \in \mathcal{M}_{\sigma}\left(\mathcal{A}^{\mathbb{Z}}\right): d_{\mathcal{M}}(\mu, \nu) \leq \varepsilon\right\},
$$


and this definition is extended to balls around a set of measures. A measure $\mu \in \mathcal{M}_{\sigma}\left(\mathcal{A}^{\mathbb{Z}}\right)$ is $\sigma$ ergodic if for every $\sigma$-invariant $B \in \mathfrak{B}\left(\sigma^{-1}(B)=B \mu\right.$-almost everywhere), one has $\mu(B)=0$ or 1. The set of $\sigma$-ergodic probability measures is denoted by $\mathcal{M}_{\sigma-\operatorname{erg}}\left(\mathcal{A}^{\mathbb{Z}}\right)$.

For $\mathbb{U} \subset \mathbb{Z}$ not necessarily finite, denote by $\mathfrak{B}_{\mathbb{U}}$ the $\sigma$-algebra generated by the set $\left\{[u]_{\mathbb{U}}: u \in\right.$ $\left.\mathcal{A}^{\mathbb{U}^{\prime}}, \mathbb{U}^{\prime} \underset{\text { finite }}{\subset} \mathbb{U}\right\}$. Define the weak mixing coefficients of a measure $\mu \in \mathcal{M}_{\sigma}\left(\mathcal{A}^{\mathbb{Z}}\right)$ as

$$
\psi_{\mu}(n)=\sup \left\{\left|\frac{\mu(A \cap B)}{\mu(A) \mu(B)}-1\right|: A \in \mathfrak{B}_{-\infty, 0]}, B \in \mathfrak{B}_{[n, \infty[}, \mu(A)>0, \mu(B)>0\right\} .
$$

A measure $\mu \in \mathcal{M}_{\sigma}\left(\mathcal{A}^{\mathbb{Z}}\right)$ is $\psi$-mixing if $\psi_{\mu}(n) \underset{n \rightarrow \infty}{\longrightarrow} 0$. Denote $\mathcal{M}_{\sigma-\text { mix }}\left(\mathcal{A}^{\mathbb{Z}}\right)$ the set of $\psi$-mixing measures. In particular $\mathcal{M}_{\sigma-\operatorname{mix}}\left(\mathcal{A}^{\mathbb{Z}}\right) \subset \mathcal{M}_{\sigma-\operatorname{erg}}\left(\mathcal{A}^{\mathbb{Z}}\right)$.

For a measure $\mu \in \mathcal{M}_{\sigma}\left(\mathcal{A}^{\mathbb{Z}}\right)$, define the support of $\mu \operatorname{supp}(\mu)$ as the set of configurations of $\mathcal{A}^{\mathbb{Z}}$ such that any open neighbourhood of these points have positive measure. $\mu$ has full support if $\operatorname{supp}(\mu)=\mathcal{A}^{\mathbb{Z}}$, which is equivalent to $\mu([u])>0$ for all $u \in \mathcal{A}^{*}$. Denote $\mathcal{M}_{\sigma-\operatorname{erg}}^{\text {full }}\left(\mathcal{A}^{\mathbb{Z}}\right)$ the set of ergodic measures with full support, and $\mathcal{M}_{\sigma-\text { mix }}^{\text {full }}\left(\mathcal{A}^{\mathbb{Z}}\right)$ the set of $\psi$-mixing measures with full support.

\subsubsection{Classical examples}

The Dirac measure supported by $x \in \mathcal{A}^{\mathbb{Z}}$ is defined as $\delta_{x}(A)=\mathbf{1}_{x \in A}$. Generally $\delta_{x}$ is not $\sigma$ invariant. However, for any $\sigma$-periodic configuration ${ }^{\infty} w^{\infty}$, it is possible to define the $\sigma$-invariant measure supported by ${ }^{\infty} w^{\infty}$ by taking the mean of the Dirac measures on the orbit under $\sigma$ :

$$
\widehat{\delta_{w}}=\frac{1}{|w|} \sum_{i \in[0,|w|-1]} \delta_{\sigma^{i}\left(\infty w^{\infty}\right)}
$$

The set of measures $\left\{\widehat{\delta_{w}}: w \in \mathcal{A}^{*}\right\}$ is dense in $\mathcal{M}_{\sigma}\left(\mathcal{A}^{\mathbb{Z}}\right)$ Pet83.

Given $\left(p_{a}\right)_{a \in \mathcal{A}}$ a family of elements of $[0,1]$ such that $\sum_{a \in \mathcal{A}} p_{a}=1$. The Bernoulli measure $\lambda_{\left(p_{a}\right)}$ associated to $\left(p_{a}\right)_{a \in \mathcal{A}}$ is defined by

$$
\lambda_{\left(p_{a}\right)}([u])=p_{u_{1}} p_{u_{2}} \ldots p_{u_{n}} \text { for all } u=u_{1} u_{2} \ldots u_{n} \in \mathcal{A}^{n} .
$$

\subsection{Action of a cellular automaton on $\mathcal{M}_{\sigma}\left(\mathcal{A}^{\mathbb{Z}}\right)$ and limit points}

\subsubsection{Definition of $\mathcal{V}(F, \mu)$ and $\mathcal{V}^{\prime}(F, \mu)$}

Let $\left(\mathcal{A}^{\mathbb{Z}}, F\right)$ be a cellular automaton and $\mu \in \mathcal{M}_{\sigma}\left(\mathcal{A}^{\mathbb{Z}}\right)$. Define the image measure $F_{*} \mu$ by $F_{*} \mu(A)=\mu\left(F^{-1}(A)\right)$ for all $A \in \mathfrak{B}$. Since $F$ is $\sigma$-invariant, that is to say $F \circ \sigma=\sigma \circ F$, one deduces that $F_{*}\left(\mathcal{M}_{\sigma}\left(\mathcal{A}^{\mathbb{Z}}\right)\right) \subset \mathcal{M}_{\sigma}\left(\mathcal{A}^{\mathbb{Z}}\right)$ and $F_{*}\left(\mathcal{M}_{\sigma-\operatorname{erg}}\left(\mathcal{A}^{\mathbb{Z}}\right)\right) \subset \mathcal{M}_{\sigma-\operatorname{erg}}\left(\mathcal{A}^{\mathbb{Z}}\right)$. This defines a continuous application $F_{*}: \mathcal{M}_{\sigma}\left(\mathcal{A}^{\mathbb{Z}}\right) \rightarrow \mathcal{M}_{\sigma}\left(\mathcal{A}^{\mathbb{Z}}\right)$.

We consider in particular $F_{*}^{t} \mu$ the iterated image of $\mu$ by $F_{*}$, and its Cesàro mean at time $t \in \mathbb{N}$ defined by $\varphi_{t}^{F}(\mu)=\frac{1}{t+1} \sum_{i=0}^{t} F_{*}^{i} \mu \in \mathcal{M}_{\sigma}\left(\mathcal{A}^{\mathbb{Z}}\right)$.

For a measure $\mu \in \mathcal{M}_{\sigma}\left(\mathcal{A}^{\mathbb{Z}}\right)$, we are interested in the asymptotic behaviour of the sequences $\left(F_{*}^{t} \mu\right)_{t \in \mathbb{N}}$ and $\left(\varphi_{t}^{F} \mu\right)_{t \in \mathbb{N}}$. Define

- $\mathcal{V}(F, \mu)$, the $\mu$-limit measures set, as the set of limit points of the sequence $\left(F^{t} \mu\right)_{t \in \mathbb{N}}$;

- $\mathcal{V}^{\prime}(F, \mu)$, the Cesàro mean $\mu$-limit measures set, as the set of limit points of the sequence $\left(\varphi_{t}^{F} \mu\right)_{t \in \mathbb{N}}$.

Since $\mathcal{M}_{\sigma}\left(\mathcal{A}^{\mathbb{Z}}\right)$ is compact, $\mathcal{V}(F, \mu)$ and $\mathcal{V}^{\prime}(F, \mu)$ are nonempty. When $\mathcal{V}(F, \mu)$ is a singleton $\{\nu\}$, $F_{*}^{t} \mu([u]) \underset{t \rightarrow \infty}{\longrightarrow} \nu([u])$. 


\subsubsection{Topological obstructions}

Our main purpose is to characterise which sets of measures can be realised in this way. There are topological obstructions for these sets: $\mathcal{V}(F, \mu)$ and $\mathcal{V}^{\prime}(F, \mu)$ are closed and thus compact since $\mathcal{M}_{\sigma}\left(\mathcal{A}^{\mathbb{Z}}\right)$ is it.

Moreover $\mathcal{V}^{\prime}(F, \mu)$ is connected. Indeed, let $\mathcal{K}$ be a connected component of $\mathcal{V}^{\prime}(F, \mu)$ and assume that $\mathcal{V}^{\prime}(F, \mu)$ is not reduced to $\mathcal{K}$. Since $\mathcal{K}$ and $\mathcal{V}^{\prime}(F, \mu) \backslash \mathcal{K}$ are closed in a compact set, there exists a minimum distance $d>0$ between them. Since $d_{\mathcal{M}}\left(\varphi_{t}^{F}(\mu), \varphi_{t+1}^{F}(\mu)\right) \underset{t \rightarrow \infty}{\longrightarrow} 0$, the set $\mathbf{B}\left(\mathcal{K}, \frac{2 d}{3}\right) \backslash \mathbf{B}\left(\mathcal{K}, \frac{d}{3}\right)$ contains arbitrarily many points in the sequence and thus contains a limit point, this is a contradiction.

\section{Computability obstructions}

In this section, we explore the computability obstructions of $\mathcal{V}(F, \mu)$ and $\mathcal{V}^{\prime}(F, \mu)$ when the initial measure $\mu$ is computable.

\subsection{Notions of computability}

Definition 1. A Turing machine $\mathcal{T} \mathcal{M}=\left(Q, \Gamma, \#, q_{0}, \delta, Q_{F}\right)$ is defined by:

- $\Gamma$ a finite alphabet and a blank symbol \# $\# \Gamma$;

- $Q$ the finite set of states of the head; $q_{0} \in Q$ is the initial state;

- $\delta: Q \times(\Gamma \cup\{\#\}) \rightarrow Q \times(\Gamma \cup\{\#\}) \times\{\leftarrow, \rightarrow\}$ the transition function.

- $Q_{F} \subset Q$ the set of final states.

Initially, a one-sided infinite memory tape is filled with \#, except for a finite prefix (the input), and a computing head in state $q_{0}$ is located on the first letter of the tape. At each time step, given the state of the head and the letter it reads on the tape - depending on its position - the head changes state, replaces the letter and moves by one cell at most, according to the transition function. When a final state is reached, the computation stops and the output is the value currently written on the tape.

A function $f: \mathcal{A}^{*} \rightarrow \mathcal{A}^{*}$ is computable if there exists a Turing machine working on an alphabet $\Gamma \supset \mathcal{A}$ that, on any input $w \in \mathcal{A}^{*}$, eventually stops and outputs $f(w)$.

To generalise this definition to functions mapping arbitrary countable sets $X \rightarrow Y$, we introduce the notion of encoding.

An encoding for a countable set $X$ is the choice of a finite alphabet $\Gamma_{X}$, a subset $V_{X} \subset \Gamma_{X}^{*}$ of valid encodings and a surjection $e_{X}: V_{X} \rightarrow X$. Intuitively, a word in $\Gamma_{X}^{*}$ represents an element of $X$, but an element can have several valid encodings and not all words of $\Gamma^{*}$ are a valid encoding of an element of $X$. Strictly speaking, the following definitions depend on the chosen encoding, but empirically all reasonable choices lead to the same notion of computability. Therefore we fix canonical encodings that will be valid throughout this article for $\mathbb{N}, \mathbb{Q}$ and their products.

- $\mathbb{N}$ or $\mathbb{Z}: \Gamma=\{0,1\}$ and to each binary sequence we assign the corresponding integer, with the first bit encoding the sign for $\mathbb{Z}$;

- $X \times Y$ : If $\Gamma_{X}, e_{X}$ and $\Gamma_{Y}, e_{Y}$ are the encodings fixed for $X$ and $Y$, respectively, we put $\Gamma=\Gamma_{X} \cup \Gamma_{Y} \sqcup\{\mid\}$ (disjoint union, i.e. assume $\mid$ is a fresh symbol), and to $x \mid y$ we assign $\left(e_{X}(x), e_{Y}(y)\right)$

- $\mathbb{Q}$ : Take the encoding for $\mathbb{Z} \times \mathbb{N}^{*}$ and compose the encoding function by $(p, q) \longmapsto \frac{p}{q}$ (surjection). 
Definition 2. A function $f: X \rightarrow Y$ between two countable sets is computable if there exists a Turing machine working on an alphabet containing $\Gamma_{X} \cup \Gamma_{Y}$ that, on any input $x \in V_{X}$, stops and outputs $y \in V_{Y}$ such that $f\left(e_{X}(x)\right)=e_{Y}(y)$.

A set $K \subset X$ is computable if $\mathbf{1}_{K}$ is computable.

\subsection{Measures and computability}

\subsubsection{Definitions and some examples}

Since a probability measure $\mu \in \mathcal{M}_{\sigma}\left(\mathcal{A}^{\mathbb{Z}}\right)$ is characterised by the value of $\mu([u])$ for all words $u \in \mathcal{A}^{*}$, it can be seen as a particular type of function $\mathcal{A}^{*} \rightarrow \mathbb{R}$. Here $\mathbb{R}$ is not countable but is a metric space with a countable dense set. Therefore it is natural to define a computable function $\mathcal{A}^{*} \rightarrow \mathbb{R}$ as a function that can be approximated by functions $\mathcal{A}^{*} \rightarrow \mathbb{Q}$ in a computable manner.

Definition 3 (Computability of probability measures). A measure $\mu \in \mathcal{M}_{\sigma}\left(\mathcal{A}^{\mathbb{Z}}\right)$ is computable (or $\Delta_{1}$-computable) iff there exists $f: \mathcal{A}^{*} \times \mathbb{N} \rightarrow \mathbb{Q}$ computable such that

$$
|\mu([u])-f(u, n)|<2^{-n} \quad \text { for all } u \in \mathcal{A}^{*} \text { and } n \in \mathbb{N} .
$$

A sequence of measures $\left(\mu_{i}\right)_{i \in \mathbb{N}}$ is a uniformly computable sequence of computable measures iff there exists $f: \mathcal{A}^{*} \times \mathbb{N} \times \mathbb{N} \rightarrow \mathbb{Q}$ computable such that $\left|\mu_{i}([u])-f(u, n, i)\right|<2^{-n}$. This is a stronger statement than saying that all $\mu_{i}$ are computable.

A measure $\mu \in \mathcal{M}_{\sigma}\left(\mathcal{A}^{\mathbb{Z}}\right)$ is limit-computable (or $\Delta_{2}$-computable) iff there exists a uniformly computable sequence of computable measures $\left(\mu_{i}\right)_{i \in \mathbb{N}}$ such that $\lim _{i \rightarrow \infty} \mu_{i}=\mu$. Equivalently there exists $f: \mathcal{A}^{*} \times \mathbb{N} \rightarrow \mathbb{Q}$ computable such that

$$
|\mu([u])-f(u, n)| \underset{n \rightarrow \infty}{\longrightarrow} 0 \quad \text { for all } u \in \mathcal{A}^{*} .
$$

Denote $\mathcal{M}_{\sigma}^{\text {comp }}\left(\mathcal{A}^{\mathbb{Z}}\right)$ the set of computable measures and $\mathcal{M}_{\sigma}^{\Delta_{2} \text {-comp }}\left(\mathcal{A}^{\mathbb{Z}}\right)$ the set of limit-computable measures. Of course $\mathcal{M}_{\sigma}^{\text {comp }}\left(\mathcal{A}^{\mathbb{Z}}\right) \subset \mathcal{M}_{\sigma}^{\Delta_{2} \text {-comp }}\left(\mathcal{A}^{\mathbb{Z}}\right)$.

Example 1. Let us provide some examples of such measures:

- any measure supported by a periodic orbit is computable;

- any Bernoulli measure or Markov measure with computable1 (resp. limit-computable) parameters is computable (resp. limit-computable);

- if an effective subshift (i.e. such the set of forbidden patterns can be enumerated by a Turing machine) has a unique $\sigma$-ergodic measure $\mu$, then $\mu$ is computable.

To obtain an approximation of $\mu([u])$ for a pattern $u$, we construct an algorithm as follows. At step $n$, the algorithm enumerates the first $n$ forbidden patterns and produces all words of size $n$ which does not contain any of these forbidden patterns. If the frequencies of $u$ in all these words are sufficiently close (less than the requested precision), the average frequency is an approximation of $\mu([u])$. If not, we continue to the step $n+1$, until an approximation is found. This process must stop since, for all elements of an unique ergodic subshift, the frequency of a pattern converges towards the measure of this pattern (we would otherwise obtain another $\sigma$-invariant measure). We deduce that $\mu$ is a computable measure. This proof can be found in GHR11 with a more abstract point of view.

This class of measures is very large. For example, this is the case for any subshift obtained by a primitive substitution or the orbit of a Sturmian word defined by some computable slope. For more details about measures of primitive substitutions and Sturmian words, see for example [FM10].

\footnotetext{
${ }^{1}$ Here the computability of a real is defined as the computability of the function that maps $n$ to the $n^{\text {th }}$ digit of the real.
} 


\subsubsection{Approximation by measures supported by periodic orbits}

It is well known that the set of measures supported by periodic orbits is dense in $\mathcal{M}_{\sigma}\left(\mathcal{A}^{\mathbb{Z}}\right)$ for the weak topology. The notions of computability for probability measures can be defined in an equivalent way using this countable dense set and the distance $d_{\mathcal{M}}$ which measures the approximation.

Proposition 1 (Approximation by measures supported by periodic orbits). It is possible to define computable and limit-computable measure as approximation of measures supported by periodic orbits.

(i) $A$ measure $\mu \in \mathcal{M}_{\sigma}\left(\mathcal{A}^{\mathbb{Z}}\right)$ is computable if and only if there exists a computable function $f: \mathbb{N} \rightarrow \mathcal{A}^{*}$ such that $d_{\mathcal{M}}\left(\mu, \widehat{\delta_{f(n)}}\right) \leq 2^{-n}$ for all $n \in \mathbb{N}$.

(ii) A measure $\mu \in \mathcal{M}_{\sigma}\left(\mathcal{A}^{\mathbb{Z}}\right)$ is limit-computable if and only if there exists a computable function $f: \mathbb{N} \rightarrow \mathcal{A}^{*}$ such that $\lim _{n \rightarrow \infty} \widehat{\delta_{f(n)}}=\mu$.

Proof. (i) Let $\mu \in \mathcal{M}_{\sigma}^{\text {comp }}\left(\mathcal{A}^{\mathbb{Z}}\right)$. Given some $n \in \mathbb{N}$, we can enumerate words in $\mathcal{A}^{*}$ in a computable manner until we find a word $f(n)$ such that $\left|\mu([u])-\widehat{\delta_{f(n)}}([u])\right|<2^{-n-2}$ for all $u \in \mathcal{A} \leq n+1$. Such a word exists since the set $\left\{\widehat{\delta_{w}}: w \in \mathcal{A}^{*}\right\}$ is dense in $\mathcal{M}_{\sigma}\left(\mathcal{A}^{\mathbb{Z}}\right)$, and it is eventually found since $\mu$ and $\widehat{\delta_{w}}$ are computable. One has

$$
d_{\mathcal{M}}\left(\mu, \widehat{\delta_{f(n)}}\right)=\sum_{i \in \mathbb{N}} \frac{1}{2^{i}} \max _{u \in \mathcal{A}^{i}}\left|\mu([u])-\widehat{\delta_{f(n)}}([u])\right| \leq \frac{1}{2^{n+1}}+\sum_{i \geq n+2} \frac{1}{2^{i}} \leq \frac{1}{2^{n}} .
$$

The converse is obvious since $(n, u) \longmapsto \widehat{\delta_{f(n)}}([u])$ is computable and we have

$$
\forall u \in \mathcal{A}^{*},\left|\mu([u])-\widehat{\delta_{f(n)}}([u])\right| \leq 2^{|u|} d_{\mathcal{M}}\left(\mu, \widehat{\delta_{f(n)}}\right) .
$$

(ii) Let $\mu \in \mathcal{M}_{\sigma}^{\Delta_{2} \text {-comp }}\left(\mathcal{A}^{\mathbb{Z}}\right)$. There exists a uniformly computable sequence of computable measures $\left(\mu_{i}\right)_{i \in \mathbb{N}}$ such that $\lim _{i \rightarrow \infty} \mu_{i}=\mu$. For each $n \in \mathbb{N}$, we enumerate words until we find $f(n) \in \mathcal{A}^{*}$ such that $d_{\mathcal{M}}\left(\mu_{n}, \widehat{\delta_{f(n)}}\right) \leq 2^{-n}$. Clearly $f: \mathbb{N} \rightarrow \mathcal{A}^{*}$ is computable and we have $d_{\mathcal{M}}\left(\mu, \widehat{\delta_{f(n)}}\right) \leq d_{\mathcal{M}}\left(\mu, \mu_{n}\right)+d_{\mathcal{M}}\left(\mu_{n}, \widehat{\delta_{f(n)}}\right) \underset{n \rightarrow \infty}{\longrightarrow} 0$. The converse is similar to the previous case.

\subsection{Action of a cellular automaton on computable measures}

When a computable measure is iterated by a cellular automaton, the resulting sequence of measures is uniformly computable. This is formalised in the following property.

Proposition 2 (Uniform computability). Let $F: \mathcal{A}^{\mathbb{Z}} \rightarrow \mathcal{A}^{\mathbb{Z}}$ be a cellular automaton. If $\mu \in$ $\mathcal{M}_{\sigma}^{\text {comp }}\left(\mathcal{A}^{\mathbb{Z}}\right)$, then $\left(F_{*}^{t} \mu\right)_{t \in \mathbb{N}}$ is an uniformly computable sequence of computable measures.

Proof. By definition, there is a computable function $f: \mathcal{A}^{*} \times \mathbb{N} \rightarrow \mathbb{Q}$ such that $|\mu([u])-f(u, n)| \leq 2^{-n}$ for all $u \in \mathcal{A}^{*}$. Because $F$ is defined locally, $F^{t}(x)_{[0, k]}$ depends only on $x_{[l t, r t+k]}$ where $l=\min \mathbb{U}_{F}$ and $r=\max \mathbb{U}_{F}$. In other words, for all $u \in \mathcal{A}^{k}$, there is a set $\operatorname{Pred}_{t}(u) \subset \mathcal{A}^{[l t, r t+k]}$ such that $F^{-t}([u])=\cup_{v \in \operatorname{Pred}_{t}(u)}[v]$. Now consider the function

$$
f^{\prime}:(u, n, t) \longmapsto \sum_{v \in \operatorname{Pred}_{t}(u)} f(v,|u|+n+(r-l) t) .
$$


It is computable by enumerating elements of $\mathcal{A}^{k+(r-l) t}$ and checking if $F^{t}\left([v]_{-l t}\right) \subset[u]$ by iterating the local rule on $v$. Finally:

$$
\begin{aligned}
\left|F_{*}^{t} \mu([u])-f^{\prime}(u, n, t)\right| & =\left|\mu\left(\bigcup_{v \in \text { Pred }_{t}(u)}[v]\right)-\sum_{v \in \text { Pred }_{t}(u)} f(v,|u|+n+(r-l) t)\right| \\
& \leq \sum_{v \in \operatorname{Pred}_{t}(u)}|\mu([v])-f(v,|u|+n+(r-l) t)| \\
& \leq 2^{|u|+(r-l) t} \cdot 2^{-|u|-n-(r-l) t}=2^{-n}
\end{aligned}
$$

which means that $\left(F_{*}^{t} \mu\right)_{t \in \mathbb{N}}$ is a uniformly computable sequence of computable measures.

From this proposition, we deduce the first computability obstruction when the sequence $\left(F_{*}^{t} \mu\right)_{t \in \mathbb{N}}$ converges toward a single limit starting from a computable measure $\mu$.

Proposition 3 (First computability obstruction). Let $F: \mathcal{A}^{\mathbb{Z}} \rightarrow \mathcal{A}^{\mathbb{Z}}$ be a cellular automaton. If $\mu \in \mathcal{M}_{\sigma}^{\text {comp }}\left(\mathcal{A}^{\mathbb{Z}}\right)$ and $F_{*}^{t} \mu \underset{t \rightarrow \infty}{\longrightarrow} \nu$ then $\nu \in \mathcal{M}_{\sigma}^{\Delta_{2}-c o m p}\left(\mathcal{A}^{\mathbb{Z}}\right)$.

We have obtained a computability obstruction on single limit measures. In the following subsections, we extend this obstruction to sets of limit points.

\subsection{Closed sets in computable analysis}

\subsubsection{Definitions and some examples}

We introduce computability notions on compact subsets of metric spaces which cannot be defined using the characteristic function since the compact set is not necessary countable. A standard reference book of the theory of computable analysis on metric spaces is [Wei00], but this theory is widely applied in the context of invariant measures (see for example GHR11]). Computability in a general metric space is defined according to a countable dense subset and a metric, $\left(\widehat{\delta_{w}}\right)_{w \in \mathcal{A}^{*}}$ and $d_{\mathcal{M}}$ in the case of $\mathcal{M}_{\sigma}\left(\mathcal{A}^{\mathbb{Z}}\right)$.

Definition 4. A closed set $\mathcal{K} \subset \mathcal{M}_{\sigma}\left(\mathcal{A}^{\mathbb{Z}}\right)$ is computable if the set

$$
\left\{(w, r) \in \mathcal{A}^{*} \times \mathbb{Q}: \overline{\mathbf{B}\left(\widehat{\delta_{w}}, r\right)} \cap \mathcal{K} \neq \emptyset\right\}
$$

is computable, that is, if its characteristic function is.

However, a set of limit points of a sequence $\left(F_{*}^{t} \mu\right)_{t \in \mathbb{N}}$, for $\mu \in \mathcal{M}_{\sigma}^{\text {comp }}\left(\mathcal{A}^{\mathbb{Z}}\right)$, is not necessarily computable. We need to extend these definitions further in order to obtain an arithmetic hierarchy.

Definition 5 ( $\Sigma_{2}$ and $\Pi_{2}$-computable function). Let $X, Y$ be two countable sets, with $Y$ being ordered. A sequence of functions $\left(f_{i}: X \rightarrow Y\right)_{i \in \mathbb{N}}\left(\right.$ resp. $\left.\left(f_{i, j}: X \rightarrow Y\right)_{i, j \in \mathbb{N}^{2}}\right)$ is uniformly computable if $(i, x) \longmapsto f_{i}(x)$ (resp. $\left.(i, j, x) \longmapsto f_{i, j}(x)\right)$ is computable.

A function $f: X \rightarrow Y$ is $\Pi_{1}$-computable (resp. $\Sigma_{1}$-computable) if $f=\inf _{i \in \mathbb{N}} f_{i}$ (resp. $\left.f=\sup _{i \in \mathbb{N}} f_{i}\right)$, where $\left(f_{i}\right)_{i \in \mathbb{N}}$ is an uniformly computable sequence of functions.

A function $f: X \rightarrow Y$ is $\Pi_{2}$-computable (resp. $\Sigma_{2}$-computable) if $f=\inf _{i \in \mathbb{N}} \sup _{j \in \mathbb{N}} f_{i, j}$ (resp. $f=\sup _{i \in \mathbb{N}} \inf _{j \in \mathbb{N}} f_{i, j}$ ), where $\left(f_{i, j}\right)_{(i, j) \in \mathbb{N}^{2}}$ is an uniformly computable sequence of functions.

Definition 6 ( $\Sigma_{2}$ and $\Pi_{2}$-computable closed set). A closed set $\mathcal{K} \subset \mathcal{M}_{\sigma}\left(\mathcal{A}^{\mathbb{Z}}\right)$ is $\Pi_{2}$-computable (resp. $\Sigma_{2}$-computable) if the set

$$
\left\{(w, r) \in \mathcal{A}^{*} \times \mathbb{Q}: \overline{\mathbf{B}\left(\widehat{\delta_{w}}, r\right)} \cap \mathcal{K} \neq \emptyset\right\}
$$

is $\Pi_{2}$-computable (resp. $\Sigma_{2}$ ), that is, if its characteristic function is. 
Remark. The symmetric notions of $\Pi_{2^{-}}$and $\Sigma_{2}$-computability come from an analogy with the real arithmetic hierarchy [ZW01, Zie05]. These definitions extend naturally to $\Pi_{n^{-}}$and $\Sigma_{n}$-computability.

\section{Example 2.}

- $\mathcal{M}_{\sigma}\left(\mathcal{A}^{\mathbb{Z}}\right)$ is a computable set.

- the set of shift-invariant measures supported by any effective subshift is a $\Pi_{1}$-computable compact set (that is to say the set $\left\{(w, r) \in \mathcal{A}^{*} \times \mathbb{Q}: \overline{\mathbf{B}\left(\widehat{\delta_{w}}, r\right)} \cap \mathcal{K} \neq \emptyset\right\}$ is $\Pi_{1}$-computable);

- let $K \subset[0,1]$ be a closed $\Pi_{n}$-computable set 2 and denote $\lambda_{p} \in \mathcal{M}_{\sigma}\left(\{0,1\}^{\mathbb{Z}}\right)$ the Bernoulli measure which charges 0 with the probability $p$ and 1 with the probability $1-p$. The set $\mathcal{K}=\left\{\lambda_{p}: p \in K\right\}$ is a $\Pi_{n}$-computable compact set of $\mathcal{M}_{\sigma}\left(\{0,1\}^{\mathbb{Z}}\right)$ and is connected if and only if $K$ is. Furthermore $\left\{\alpha \lambda_{p}+(1-\alpha) \lambda_{q}: p, q \in K\right.$ and $\left.\alpha \in[0,1]\right\}$ is a $\Pi_{n}$-computable compact connected set of $\{0,1\} \mathbb{Z}$. This example extends naturally to larger alphabets and Markov measures;

- denote $\mu_{\alpha} \in \mathcal{M}_{\sigma}\left(\{0,1\}^{\mathbb{Z}}\right)$ the measure supported by the Sturmian subshift of slope $\alpha$. The set $\mathcal{K}=\left\{\mu_{\alpha}: \alpha \in K\right\}$, where $K$ is a $\Pi_{n}$-computable closed subset of $[0,1]$, is a $\Pi_{n}$-computable compact set of $\mathcal{M}_{\sigma}\left(\{0,1\}^{\mathbb{Z}}\right)$ and is connected if and only if $K$ is.

Proof of the second example. Let $\Sigma \subset \mathcal{A}^{\mathbb{Z}}$ be an effective subshift, which means that it is defined by a set of forbidden patterns $\mathcal{F}$ and there exists a computable function $f: i, u \longmapsto\{0,1\}$ such that $u \in \mathcal{F} \Longleftrightarrow \sup _{i} f(i, u)=1$. Denote $\mathcal{F}_{i}=\left\{u \in \mathcal{A}^{*}: \sup _{j \leq i} f(j, u)=1\right\}$.

There exists an effective sequence of integers $\left(\alpha_{i}\right)_{i \in \mathbb{N}}$ such that:

$$
\forall \mu \in \mathcal{M}_{\sigma}\left(\mathcal{A}^{\mathbb{Z}}\right), \exists w \in \mathcal{A}^{\leq \alpha_{i}}, d_{\mathcal{M}}\left(\mu, \widehat{\delta_{w}}\right) \leq \frac{1}{i} .
$$

This is due from the fact that $\mathcal{M}_{\sigma}\left(\mathcal{A}^{\mathbb{Z}}\right)$ is a recursively precompact metric space (see GHR11]).

Now define:

$$
\mathbf{W}_{i}=\left\{w \in \mathcal{A}^{\leq \alpha_{i}}: \sum_{\ell \in \mathbb{N}} \frac{1}{2^{\ell}} \max _{v \in \mathcal{F}_{i} \cap \mathcal{A}^{\ell}} \widehat{\delta_{w}}([v]) \leq \frac{1}{i}\right\},
$$

where the maximum is worth 0 when the set is empty, which means that the sum has a finite number of terms: the maximum length of a word in $\mathcal{F}_{i}$.

Let $A$ be the algorithm that, on input $(u, r) \in \mathcal{A}^{*} \times \mathbb{Q}$ and $i \in \mathbb{N}$,

(1) computes all elements of $\mathcal{F}_{i}$ (evaluating a computable function over a finite set of arguments);

(2) computes all $w \in \mathbf{W}_{i}$ (a finite number of tests, and $u \longmapsto \widehat{\delta_{w}}\left([u]\right.$ ) is a function $\mathcal{A}^{*} \rightarrow \mathbb{Q}$ which can be evaluated exactly);

(3) computes $d_{i}\left(\widehat{\delta_{w}}, \widehat{\delta_{u}}\right)=\sum_{n=0}^{i} \frac{1}{2^{n}} \max _{v \in \mathcal{A}^{n}}\left|\widehat{\delta_{w}}([v])-\widehat{\delta_{u}}([v])\right|$ for all $w \in \mathbf{W}_{i}$;

(4) outputs 1 if there exists $w \in \mathbf{W}_{i}$ such that $d_{i}\left(\widehat{\delta_{w}}, \widehat{\delta_{u}}\right) \leq r+\frac{1}{i}$ and 0 otherwise.

We prove the correctness of this algorithm, that is, we show that

$$
\inf _{i \in \mathbb{N}} A(u, r, i)=1 \Longleftrightarrow \overline{\mathbf{B}\left(\widehat{\delta_{u}}, r\right)} \cap \mathcal{M}_{\sigma}(\Sigma) \neq \emptyset .
$$

Notice that for every sequence $\left(w_{i}\right)_{i \in \mathbb{N}}$ satisfying $w_{i} \in \mathbf{W}_{i}$ for all $i$, any accumulation point $\mu$ of the sequence $\left(\widehat{\delta_{w_{i}}}\right)_{i \in \mathbb{N}}$ satisfies $\mu([u])=0$ for all $u \in \mathcal{F}$, and therefore $\mu \in \mathcal{M}_{\sigma}(\Sigma)$.

If $\inf _{i \in \mathbb{N}} A(u, r, i)=1$ then for all $i \in \mathbb{N}$ there exists $w_{i} \in \mathbf{W}_{i}$ such that $d_{i}\left(\widehat{\delta_{w_{i}}}, \widehat{\delta_{u}}\right) \leq r+\frac{1}{i}$. Thus one has $d_{\mathcal{M}}\left(\widehat{\delta_{w_{i}}}, \widehat{\delta_{u}}\right) \leq r+\frac{1}{2^{i}}+\frac{1}{i}$. We deduce that for any accumulation point $\mu$ of $\left(\widehat{\delta_{w_{i}}}\right)_{i \in \mathbb{N}}$ one has $d_{\mathcal{M}}\left(\mu, \widehat{\delta_{u}}\right) \leq r$ and therefore $\overline{\mathbf{B}\left(\widehat{\delta_{u}}, r\right)} \cap \mathcal{M}_{\sigma}(\Sigma) \neq \emptyset$.

\footnotetext{
${ }^{2}$ The computability of a closed set of real numbers is defined similarly to the computability of a closed set of probability measures.
} 
Conversely, let $\mu \in \overline{\mathbf{B}\left(\widehat{\delta_{u}}, r\right)} \cap \mathcal{M}_{\sigma}(\Sigma)$. There exists a sequence $\left(w_{i}\right)_{i \in \mathbb{N}}$ such that $w_{i} \in \mathcal{A} \leq \alpha_{i}$ and $d_{\mathcal{M}}\left(\mu, \widehat{\delta_{w_{i}}}\right) \leq \frac{1}{i}$. Since $\mu \in \mathcal{M}_{\sigma}(\Sigma)$, one has

$$
\frac{1}{i} \geq d_{\mathcal{M}}\left(\mu, \widehat{\delta_{w_{i}}}\right) \geq \sum_{\ell \in \mathbb{N}} \max _{u \in \mathcal{F} \cap \mathcal{A}^{\ell}} \widehat{\delta_{w_{i}}}([u]) \geq \sum_{\ell \in \mathbb{N}} \max _{u \in \mathcal{F}_{i} \cap \mathcal{A}^{\ell}} \widehat{\delta_{w_{i}}}([u])
$$

which means that $w_{i} \in \mathbf{W}_{i}$. Furthermore,

$$
d_{i}\left(\widehat{\delta_{u}}, \widehat{\delta_{w_{i}}}\right) \leq d_{\mathcal{M}}\left(\widehat{\delta_{u}}, \widehat{\delta_{w_{i}}}\right) \leq d_{\mathcal{M}}\left(\widehat{\delta_{u}}, \mu\right)+d_{\mathcal{M}}\left(\mu, \widehat{\delta_{w_{i}}}\right) \leq r+\frac{1}{i}
$$

so $A(u, r, i)=1$ for all $i \in \mathbb{N}$.

We conclude that $\mathcal{M}_{\sigma}(\Sigma)$ is a $\Pi_{1}$-computable set.

\subsubsection{Equivalent definitions of $\Pi_{2}$-computability}

The $\Pi_{2}$-computability of a closed set can be defined in other equivalent ways, which requires to extend notions of computability and $\Pi_{2}$-computability to functions mapping metric spaces with countable dense sets.

Definition 7. A sequence of functions $\left(f_{n}: \mathcal{M}_{\sigma}\left(\mathcal{A}^{\mathbb{Z}}\right) \longrightarrow \mathbb{R}\right)_{n \in \mathbb{N}}$ is a uniformly computable sequence of functions if:

- there exists $a: \mathbb{N} \times \mathbb{N} \times \mathcal{A}^{*} \longrightarrow \mathbb{Q}$ computable such that $\left|f_{n}\left(\widehat{\delta_{w}}\right)-a(n, m, w)\right| \leq \frac{1}{m}$ for every $w \in \mathcal{A}^{*}$ and $n, m \in \mathbb{N}$ (sequential computability);

- there exists $b: \mathbb{N} \longrightarrow \mathbb{Q}^{+}$computable such that $d_{\mathcal{M}}(\mu, \nu) \leq b(m)$ implies $\left|f_{n}(\mu)-f_{n}(\nu)\right| \leq$ $\frac{1}{m}$ for all $n, m \in \mathbb{N}$ (computable uniform equicontinuity).

A function $f: \mathcal{M}_{\sigma}\left(\mathcal{A}^{\mathbb{Z}}\right) \longrightarrow \mathbb{R}$ is $\Pi_{1}$-computable if there exists a uniformly computable sequence of functions $\left(f_{n}: \mathcal{M}_{\sigma}\left(\mathcal{A}^{\mathbb{Z}}\right) \longrightarrow \mathbb{R}\right)_{n \in \mathbb{N}}$ such that $f=\inf _{n} f_{n}$.

A function $f: \mathcal{M}_{\sigma}\left(\mathcal{A}^{\mathbb{Z}}\right) \longrightarrow \mathbb{R}$ is $\Sigma_{2}$-computable if there exists a uniformly computable sequence of computable functions $\left(f_{i, j}: \mathcal{M}_{\sigma}\left(\mathcal{A}^{\mathbb{Z}}\right) \longrightarrow \mathbb{R}\right)_{(i, j) \in \mathbb{N}} \operatorname{such}_{\text {that }} f=\sup _{i} \inf _{j} f_{i, j}$.

Proposition 4. Let $\mathcal{K} \subset \mathcal{M}_{\sigma}\left(\mathcal{A}^{\mathbb{Z}}\right)$ be a closed set. The following are equivalent:

(1) $\mathcal{K}$ is $\Pi_{2}$-computable;

(2) $d_{\mathcal{K}}: \mu \longmapsto \min _{\nu \in \mathcal{K}} d_{\mathcal{M}}(\mu, \nu)$ is $\Sigma_{2}$-computable;

(3) $\mathcal{K}=f^{-1}(\{0\})$ where $f: \mathcal{M}_{\sigma}\left(\mathcal{A}^{\mathbb{Z}}\right) \longrightarrow \mathbb{R}$ is a $\Pi_{1}$-computable function.

Proof.

$\mathbf{1} \Longrightarrow \mathbf{2}$ : Assume there is a computable function $f: \mathbb{N}^{2} \times \mathcal{A}^{*} \times \mathbb{Q} \longrightarrow \mathbb{R}$ such that, for every $w \in \mathcal{A}^{*}$ and $r \in \mathbb{Q}, \overline{\mathbf{B}\left(\widehat{\delta_{w}}, r\right)} \cap \mathcal{K} \neq \emptyset \Longleftrightarrow \inf _{i} \sup _{j} f(i, j, w, r)=1$. Consider the sequence

$$
\left(d_{i, j, w, r}: \mu \longmapsto(1-f(i, j, w, r)) \max \left(0, r-d_{\mathcal{M}}\left(\widehat{\delta_{w}}, \mu\right)\right)\right)_{(i, j, w, r) \in \mathbb{N}^{2} \times \mathcal{A}^{*} \times \mathbb{Q}} .
$$

The function $\left(i, j, w, r, w^{\prime}\right) \longmapsto d_{i, j, w, r}\left(\widehat{\delta_{w^{\prime}}}\right)$ is computable as a product of computable functions (sequential computability) and every $d_{i, j, w, r}$ is 1-Lipschitz (computable uniform equicontinuity), hence this sequence is a uniformly computable sequence of functions. We now show that $d_{\mathcal{K}}=$ $\sup _{w, r} \sup _{i} \inf _{j} d_{i, j, w, r}$.

For any $(w, r)$ such that $\inf _{i} \sup _{j} f(i, j, w, r)=0$, we have $d_{\mathcal{K}}\left(\widehat{\delta_{w}}\right)>r$, and thus for all $\mu \in$ $\mathcal{M}_{\sigma}\left(\mathcal{A}^{\mathbb{Z}}\right)$ one has:

$$
\sup _{i} \inf _{j} d_{i, j, w, r}(\mu)=\max \left(0, r-d_{\mathcal{M}}\left(\widehat{\delta_{w}}, \mu\right)\right) \leq \max \left(0, d_{\mathcal{K}}\left(\widehat{\delta_{w}}\right)-d_{\mathcal{M}}\left(\widehat{\delta_{w}}, \mu\right)\right) \leq d_{\mathcal{K}}(\mu) .
$$

If $\mu \in \mathcal{K}$, we conclude that $\sup _{i, w, r} \inf _{j} d_{i, j, w, r}(\mu)=0=d_{\mathcal{K}}(\mu)$. 
Now let $\mu \notin \mathcal{K}$. For all $\varepsilon>0$, there exists $w$ such that $d_{\mathcal{M}}\left(\widehat{\delta_{w}}, \mu\right) \leq \varepsilon$. Let $r \in \mathbb{Q}$ be such that $0<d_{\mathcal{K}}\left(\widehat{\delta_{w}}\right)-r<\varepsilon$, which implies that $\widehat{\mathbf{B}\left(\widehat{\delta_{w}}, r\right)} \cap \mathcal{K}=\emptyset$ and $\operatorname{so}_{\inf _{i}} \sup _{j} f(i, j, w, r)=0$. Furthermore $d_{\mathcal{K}}(\mu) \leq d_{\mathcal{K}}\left(\widehat{\delta_{w}}\right)+d_{\mathcal{M}}\left(\widehat{\delta_{w}}, \mu\right) \leq r+2 \varepsilon$, we deduce that

$$
\sup _{i} \inf _{j} d_{i, j, w, r}(\mu)=r-d_{\mathcal{M}}\left(\widehat{\delta_{w}}, \mu\right) \leq r-2 \varepsilon-d_{\mathcal{M}}\left(\widehat{\delta_{w}}, \mu\right) \leq d_{\mathcal{K}}(\mu)-3 \varepsilon .
$$

The latter is true for every $\varepsilon>0$, we deduce that $\sup _{i, w, r} \inf _{j} d_{i, j, w, r}(\mu)=d_{\mathcal{K}}(\mu)$.

$\mathbf{2} \Longrightarrow \mathbf{3}$ : Let $\left(d_{i, j}: \mathcal{M}_{\sigma}\left(\mathcal{A}^{\mathbb{Z}}\right) \longrightarrow \mathbb{R}\right)_{(i, j) \in \mathbb{N}^{2}}$ be a uniformly computable sequence of computable functions such that $d_{\mathcal{K}}=\sup _{i \in \mathbb{N}} \inf _{j \in \mathbb{N}} d_{i, j}$. By considering $\sup \left(d_{i, j}, 0\right)$ (which is uniformly computable since $x \longmapsto \sup (x, 0)$ is computable), these functions are assumed nonnegative w.l.o.g. Denote $g_{i, n}=\inf \left\{d_{i, j}: j \in\{0, \ldots, n\}\right\}$.

$$
\begin{aligned}
d_{\mathcal{K}}(\mu)=0 & \Longleftrightarrow \sum_{i \in \mathbb{N}} \frac{1}{2^{i}}\left(\inf _{j \in \mathbb{N}} d_{i, j}(\mu)\right)=0 \\
& \Longleftrightarrow \sum_{i \in \mathbb{N}} \frac{1}{2^{i}}\left(\inf _{n \in \mathbb{N}} g_{i, n}(\mu)\right)=0 \\
& \Longleftrightarrow \inf _{n \in \mathbb{N}} \sum_{i \in \mathbb{N}} \frac{1}{2^{i}} g_{i, n}(\mu)=0,
\end{aligned}
$$

where the last equivalence is obtained by the monotone convergence theorem, $g_{i, n}$ being decreasing in $n$. Let $f_{n}: \mu \longmapsto \sum_{i \in \mathbb{N}} \frac{1}{2^{2}} g_{i, n}(\mu)$. $\left(f_{n}\right)_{n \in \mathbb{N}}$ is a uniformly computable sequence of functions, since computing $\left(n, w^{\prime}\right) \longmapsto f_{n}\left(\widehat{\delta_{w^{\prime}}}\right)$ up to precision $2^{-r}$ only requires to compute the values of $d_{i, j}\left(\widehat{\delta_{w^{\prime}}}\right)$ for $i, j \in\{0, \ldots, r\}$, and the computable uniform equicontinuity of $\left(f_{n}\right)_{n \in \mathbb{N}}$ is a consequence of the computable uniform equicontinuity of $\left(d_{i, j}\right)_{(i, j) \in \mathbb{N}^{2}}$. Thus $\mathcal{K}=f^{-1}(0)$ where $f=\inf _{n} f_{n}$.

$\mathbf{3} \Longrightarrow \mathbf{1}:$ Let $\left(f_{n}: \mathcal{M}_{\sigma}\left(\mathcal{A}^{\mathbb{Z}}\right) \rightarrow \mathbb{R}\right)_{n \in \mathbb{N}}$ be a uniformly computable sequence of functions such that $f=\inf _{n \in \mathbb{N}} f_{n}$. We assume w.l.o.g that the sequence is decreasing. For $i \in \mathbb{N}$, we note:

$$
d_{i}(\mu, \nu)=\sum_{n=1}^{i} \frac{1}{2^{n}} \max _{u \in \mathcal{A}^{n}}|\mu([u])-\nu([u])|,
$$

so that $0 \leq d_{\mathcal{M}}(\mu, \nu)-d_{i}(\mu, \nu) \leq \frac{1}{2^{i}}$. For any $n \in \mathbb{N}, w^{\prime} \in \mathcal{A}^{*}$ and $i \in \mathbb{N}$, define

$$
K_{n, w^{\prime}, i}=\left\{(w, r) \in \mathcal{A}^{*} \times \mathbb{Q}: d_{i}\left(\widehat{\delta_{w}}, \widehat{\delta_{w^{\prime}}}\right) \leq r \text { and }\left|f_{i}\left(\widehat{\delta_{w^{\prime}}}\right)\right| \leq \frac{1}{n}\right\}
$$

The function $\left(n, w^{\prime}, i, w, r\right) \longmapsto \mathbf{1}_{K_{n, w^{\prime}, i}}(w, r)$ is computable and thus the characteristic functions $\mathbf{1}_{K_{n, w^{\prime}, i}}$ are uniformly computable. Define:

$$
K=\bigcap_{n \in \mathbb{N}} \bigcup_{\substack{w^{\prime} \in \mathcal{A}^{*} \\ i \in \mathbb{N}}} K_{n, w^{\prime}, i} \quad \text { and thus } \quad \mathbf{1}_{K}=\inf _{n \in \mathbb{N}} \sup _{\substack{w^{\prime} \in \mathcal{A}^{*} \\ i \in \mathbb{N}}} \mathbf{1}_{K_{n, w^{\prime}, i}} .
$$

The set $K$ is $\Pi_{2}$-computable by definition, we are going to prove that

$$
K=\left\{(w, r) \in \mathcal{A}^{*} \times \mathbb{Q}: \overline{\mathbf{B}\left(\widehat{\delta_{w}}, r\right)} \cap \mathcal{K} \neq \emptyset\right\} .
$$

Let $(w, r) \in K$. For all $n \in \mathbb{N}$, there exists $w_{n} \in \mathcal{A}^{*}$ and $i_{n} \in \mathbb{N}$ such that $d_{\mathcal{M}}\left(\widehat{\delta_{w}}, \widehat{\delta_{w_{n}}}\right) \leq r$ and $\left|f_{i_{n}}\right| \leq \frac{1}{n}$. By compactness, there exists $\mu \in \mathcal{M}_{\sigma}\left(\mathcal{A}^{\mathbb{Z}}\right)$ such that $d_{\mathcal{M}}\left(\widehat{\delta_{w}}, \mu\right) \leq r$ and $f(\mu)=$ $\lim _{n \rightarrow \infty} f_{i_{n}}\left(w_{i_{n}}\right)=0$. Thus $\overline{\mathbf{B}\left(\widehat{\delta_{w}}, r\right)} \cap \mathcal{K} \neq \emptyset$. 
Conversely, consider $\mu \in \overline{\mathbf{B}\left(\widehat{\delta_{w}}, r\right)} \cap \mathcal{K}$. Since $f(\mu)=0$, for all $n \in \mathbb{N}$ there exists $i_{n} \in \mathbb{N}$ such that $\left|f_{i_{n}}(\mu)\right| \leq \frac{1}{2 n}$. Thus one has $\left|f_{i_{n}}(\nu)\right| \leq \frac{1}{n}$ for all $\nu \in \mathbf{B}(\mu, b(2 n))$. Let $w_{n} \in \mathcal{A}^{*}$ such that $\widehat{\delta_{w_{n}}} \in \widehat{\mathbf{B}\left(\widehat{\delta_{w}}, r\right)} \cap \mathbf{B}(\mu, b(n))$. One has $\left|f_{i_{n}}\left(w_{n}\right)\right| \leq \frac{1}{n}$, that is to say $(w, r) \in K_{n, w_{n}, i_{n}}$. Since it is verified for all $n \in \mathbb{N}$, one deduces that $(w, r) \in K$.

Remark. There exist other equivalent definitions for $\Pi_{2}$-computable closed set. See [dM14 for some complements.

\subsection{Computability obstruction for $\mathcal{V}(F, \mu)$ and $\mathcal{V}^{\prime}(F, \mu)$}

We now state the computability obstruction for subsets of $\mathcal{M}_{\sigma}\left(\mathcal{A}^{\mathbb{Z}}\right)$ reachable as limit sets of the sequence $\left(F_{*}^{t} \mu\right)_{t \in \mathbb{N}}(\mu$-limit measures sets).

Proposition 5 (Second computability obstruction). Let $F: \mathcal{A}^{\mathbb{Z}} \rightarrow \mathcal{A}^{\mathbb{Z}}$ be a cellular automaton and $\mu \in \mathcal{M}_{\sigma}^{\text {comp }}\left(\mathcal{A}^{\mathbb{Z}}\right)$. Then $\mathcal{V}(F, \mu)$ and $\mathcal{V}^{\prime}(F, \mu)$ are nonempty $\Pi_{2}$-computable compact sets.

Remark. If a $\Pi_{2}$-computable closed set of measures is reduced to a single measure, then this measure is limit-computable. Thus Proposition 5 implies Proposition 3 .

Proof. Let $f_{n}: \nu \longmapsto d_{\mathcal{M}}\left(F_{*}^{n} \mu, \nu\right)$. Since $\mu$ is computable, $\left(f_{n}\right)_{n \in \mathbb{N}}$ is sequentially computable. Moreover $\left|f_{n}(\nu)-f_{n}\left(\nu^{\prime}\right)\right|=\left|d_{\mathcal{M}}\left(F_{*}^{n} \mu, \nu\right)-d_{\mathcal{M}}\left(F_{*}^{n} \mu, \nu^{\prime}\right)\right| \leq d_{\mathcal{M}}\left(\nu, \nu^{\prime}\right)$ so $\left(f_{n}\right)_{n \in \mathbb{N}}$ is computably uniformly equicontinuous. The result follows from the fact that $d_{\mathcal{V}(F, \mu)}(\nu)=\liminf _{n \rightarrow \infty} d_{\mathcal{M}}\left(F_{*}^{n} \mu, \nu\right)=$ $\sup _{m} \inf _{n>m} f_{n}(\nu)$, using Proposition 4.

The same reasoning holds for $\mathcal{V}^{\prime}(F, \mu)$.

Remark. When the initial measure is not computable, it can be used as an oracle. These obstructions will be generalised accordingly in Section 5.4.

\subsection{Technical characterisation of $\Pi_{2}$-computable compact connected sets}

$\Pi_{2}$-computable compact set of measures can be described as the limit points of a sequence $\left(\widehat{\delta_{w_{n}}}\right)_{n \in \mathbb{N}}$ corresponding to some uniformly computable sequence of words $\left(w_{n}\right)_{n \in \mathbb{N}}$. However, for technical reasons, the $\mu$-limit measures set of the construction presented in Section 3 corresponds to the limit set of an infinite polygonal path composed of segments of the form

$$
\left[\widehat{\delta_{u}}, \widehat{\delta_{v}}\right]=\left\{t \widehat{\delta_{u}}+(1-t) \widehat{\delta_{v}}: t \in[0,1]\right\} \subset \mathcal{M}_{\sigma}\left(\mathcal{A}^{\mathbb{Z}}\right)
$$

where $u, v \in \mathcal{A}^{*}$, and is in particular connected. This is why we describe in the following proposition how compact, $\Pi_{2}$-computable, connected sets can be covered by a polygonal path corresponding to a uniformly computable sequence of words.

Definition 8. Let $\left(w_{n}\right)_{n \in \mathbb{N}}$ be a sequence of words of $A^{*}$. Denote $\mathcal{V}\left(\left(w_{n}\right)_{n \in \mathbb{N}}\right)$ the limit points of the polygonal path defined by the sequence of measures $\left(\widehat{\delta_{w_{n}}}\right)_{n \in \mathbb{N}}$ :

$$
\mathcal{V}\left(\left(w_{n}\right)_{n \in \mathbb{N}}\right)=\bigcap_{N>0} \widehat{\bigcup_{n \geq N}\left[\widehat{\delta_{w_{n}}}, \widehat{\delta_{w_{n+1}}}\right]} .
$$

Proposition 6. Let $\mathcal{K} \subset \mathcal{M}_{\sigma}\left(\mathcal{A}^{\mathbb{Z}}\right)$ be a non-empty $\Pi_{2}$-computable, compact, connected set $\left(\Pi_{2}\right.$ $C C C$ for short). Then there exists a uniformly computable sequence of words $\left(w_{n}\right)_{n \in \mathbb{N}}$ such that $\mathcal{K}=\mathcal{V}\left(\left(w_{n}\right)_{n \in \mathbb{N}}\right)$.

Proof. By Proposition 4 there is a uniformly computable sequence of functions $\left(f_{n}\right)_{n \in \mathbb{N}}$ satisfying $\mathcal{K}=f^{-1}(\{0\})$ where $f=\inf _{n \in \mathbb{N}} f_{n}$. Let $a: \mathbb{N} \times \mathbb{N} \times \mathcal{A}^{*} \rightarrow \mathbb{Q}$ and $b: \mathbb{N} \rightarrow \mathbb{Q}^{+}$be the computable 
functions given by Definition 7 . Without loss of generality, we can assume that $b$ is a decreasing function and $b(i) \underset{i \rightarrow \infty}{\longrightarrow} 0$.

For $k \in \mathbb{N}$, define:

$$
\begin{gathered}
\alpha_{k}^{t}=\min \left\{\ell \leq t: \forall u \in \mathcal{A}^{\leq t}, \exists w \in \mathcal{A}^{\leq \ell}, d_{\mathcal{M}}\left(\widehat{\delta_{u}}, \widehat{\delta_{w}}\right) \leq b(k)\right\} \\
\alpha_{k}=\min \left\{\ell \in \mathbb{N}: \mathcal{M}_{\sigma}\left(\mathcal{A}^{\mathbb{Z}}\right)=\bigcup_{u \in \mathcal{A} \leq \ell} \mathbf{B}\left(\widehat{\delta_{u}}, b(k)\right)\right\} \\
\mathbf{V}_{k}^{t}=\left\{u \in \mathcal{A}^{\leq \alpha_{k}^{t}}: \exists n \leq t \text { such that } a(n, k, u)<\frac{2}{k}\right\} \\
\mathbf{V}_{k}=\left\{u \in \mathcal{A}^{\leq \alpha_{k}}: \exists n \in \mathbb{N} \text { such that } a(n, k, u)<\frac{2}{k}\right\}
\end{gathered}
$$

Since the periodic measures are dense in $\mathcal{M}_{\sigma}\left(\mathcal{A}^{\mathbb{Z}}\right)$, we have $\alpha_{k}^{t}=\alpha_{k}$ when $t$ is large enough. Furthermore $\alpha_{k} \underset{k \rightarrow+\infty}{\longrightarrow}+\infty$.

Moreover, for $u \in \mathcal{A} \leq \alpha_{k}$, if $f\left(\widehat{\delta_{u}}\right)<\frac{1}{k}$ there exists $n \in \mathbb{N}$ such that $f_{n}\left(\widehat{\delta_{u}}\right)<\frac{1}{k}$ so $a(n, k, u)<\frac{2}{k}$ which implies that $u \in \mathbf{V}_{k}$. Conversely, if $u \in \mathbf{V}_{k}$ then there exists $n \in \mathbb{N}$ such that $a(n, k, u)<\frac{2}{k}$ so $f\left(\widehat{\delta_{u}}\right) \leq f_{n}\left(\widehat{\delta_{u}}\right) \leq a(n, k, u)+\frac{1}{k} \leq \frac{3}{k}$.

Claim 1: $\quad \mathbf{V}_{k}^{t}$ is increasing with regards to $t$ and there exists $T_{k}$ such that $\mathbf{V}_{k}^{T_{k}}=\mathbf{V}_{k}$. Furthermore, the function $(k, t, w) \rightarrow 1_{\mathbf{V}_{k}^{t}}(w)$ is computable.

Proof. For all $k$ and $t, \mathbf{V}_{k}^{t} \subset \mathbf{V}_{k}^{t+1}$. Furthermore, if $w \in \mathbf{V}_{k}$, then $w \in \mathbf{V}_{k}^{t}$ for $t$ large enough. Since $\mathbf{V}_{k}$ is finite, there is a $T_{k}$ such that $\mathbf{V}_{k}=\mathbf{V}_{k}^{T_{k}}$.

The conditions for being included in $\mathbf{V}_{k}^{t}$ can be checked by computing computable functions over a finite range of values, so $(k, t, w) \longmapsto 1_{\mathbf{V}_{k}^{t}}(w)$ is computable.

Notice that the $T_{k}$ are not necessarily computable, which means that even though each $\mathbf{V}_{k}$ is finite, there is not necessarily a way to know when the enumeration is finished.

Claim 2:

$$
\mathcal{K}=\bigcap_{k} \bigcup_{u \in \mathbf{V}_{k}} \mathbf{B}\left(\widehat{\delta_{u}}, b(k)\right) .
$$

Proof. For each element $\mu \in \mathcal{K}$ and $k \in \mathbb{N}$, there is an element $u_{k} \in \mathcal{A}^{\leq \alpha_{k}}$ such that $d_{\mathcal{M}}\left(\mu, \widehat{\delta_{u_{k}}}\right) \leq b(k)$, and therefore $f\left(\widehat{\delta_{u_{k}}}\right) \leq \frac{1}{k}$. Thus, there is $m \in \mathbb{N}$ such that $f_{m}\left(\widehat{\delta_{u_{k}}}\right)<\frac{1}{k}$. One deduces that $a\left(m, k, u_{k}\right) \leq f_{m}\left(\widehat{\delta_{u_{k}}}\right)+\frac{1}{k}<\frac{2}{k}$, which means that $u_{k} \in \mathbf{V}_{k}$. In other words,

$$
\forall k \in \mathbb{N}, \mathcal{K} \subset \bigcup_{u \in \mathbf{V}_{k}} \mathbf{B}\left(\widehat{\delta_{u}}, b(k)\right) \text {. }
$$

Conversely, let $\mu \in \bigcap_{k} \bigcup_{u \in \mathbf{V}_{k}} \mathbf{B}\left(\widehat{\delta_{u}}, b(k)\right)$. For all $k \in \mathbb{N}$, there exists $u_{k} \in \mathbf{V}_{k}$ such that $\mu \in \mathbf{B}\left(\widehat{\delta_{u_{k}}}, b(k)\right)$. Following the same reasoning as before, there exists $n \in \mathbb{N}$ such that $a\left(n, k, u_{k}\right) \leq \frac{2}{k}$ and so

$$
f(\mu) \leq f\left(\widehat{\delta_{u_{k}}}\right)+\frac{1}{k} \leq f_{n}\left(\widehat{\delta_{u_{k}}}\right)+\frac{1}{k} \leq a\left(n, k, u_{k}\right)+\frac{2}{k} \leq \frac{4}{k}
$$

We conclude that $f(\mu)=0$ so $\mu \in \mathcal{K}$. 
We introduce Algorithm 1 for computing the sequence $\left(w_{n}\right)_{n \in \mathbb{N}}$ which realizes $\mathcal{K}$ as the limit points of the polygonal path defined by $\left(\widehat{\delta_{w_{n}}}\right)_{n \in \mathbb{N}}$.

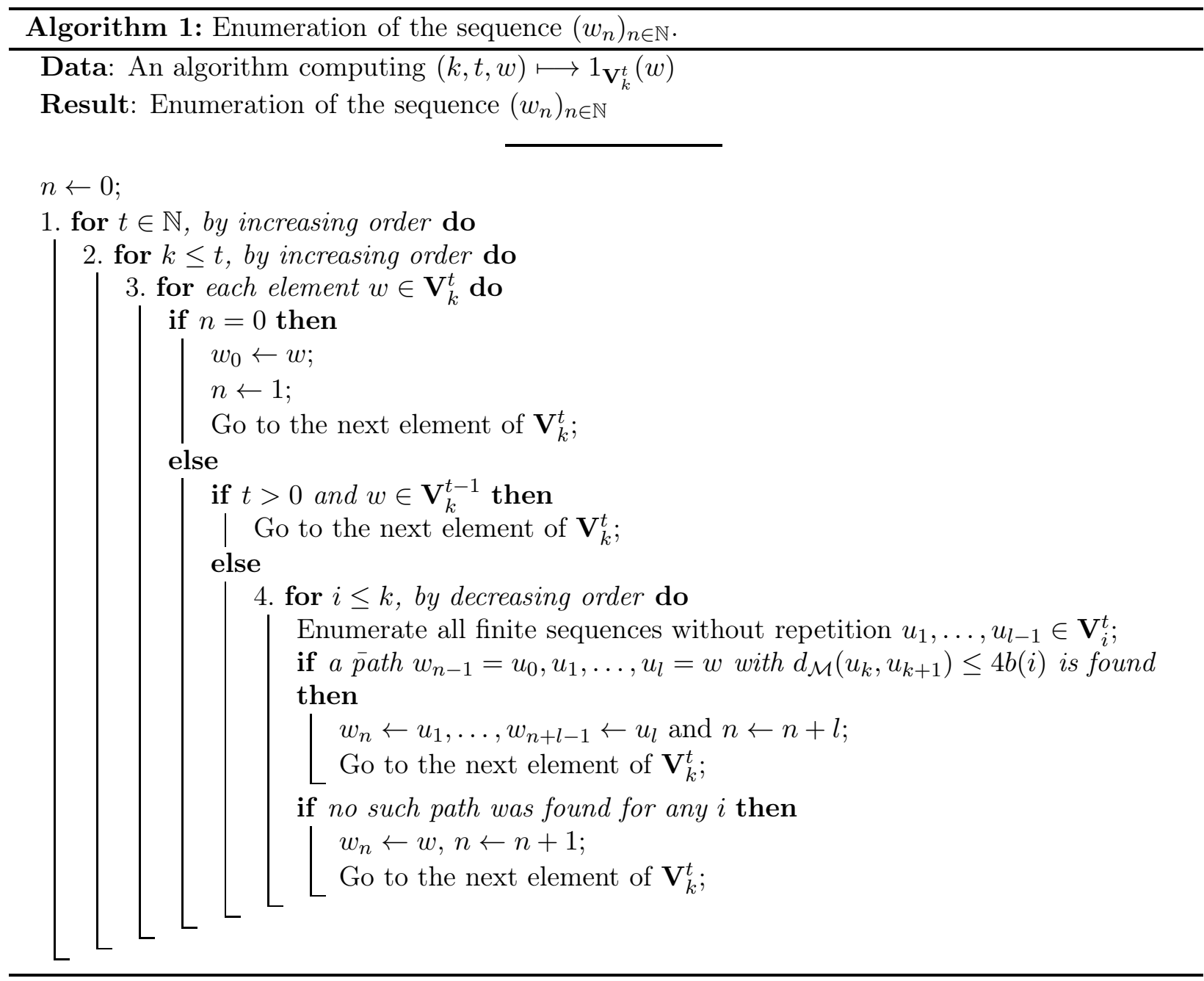

Notice that in the fourth loop, if a path is found, then it corresponds to the largest $i \leq k$ for which such a path exists. Now we prove the correctness of this algorithm. First notice that all elements of all $\mathbf{V}_{k}$ is enumerated in $\left(w_{n}\right)_{n \in \mathbb{N}}$.

Claim 3: If $\mu \in \mathcal{K}$, then $\mu \in \mathcal{V}\left(\left(w_{n}\right)_{n \in \mathbb{N}}\right)$.

Proof. By Claim 2, there is a sequence of words $\left(u_{k}\right)_{k \in \mathbb{N}}$ such that $u_{k} \in \mathbf{V}_{k}$ and $d_{\mathcal{M}}\left(\widehat{\delta_{u_{k}}}, \mu\right)<$ $b(k)$ for all $k \in \mathbb{N}$. So $u_{k}$ appears at some point in the sequence $\left(w_{n}\right)_{n \in \mathbb{N}}$ for every $k \in \mathbb{N}$. We conclude that $\lim _{k \rightarrow \infty} \widehat{\delta_{u_{k}}}=\mu \in \mathcal{V}\left(\left(w_{n}\right)_{n \in \mathbb{N}}\right) . \quad \quad \diamond$ claim 3

Claim 4: For every $\varepsilon>0$, there exists a $t_{\varepsilon}$ such that in the previous algorithm, if $t^{\prime} \geq t \geq t_{\varepsilon}$, $w \in \mathbf{V}_{k}^{t+1} \backslash \mathbf{V}_{k}^{t}$ and $w^{\prime} \in \mathbf{V}_{k^{\prime}}^{t^{\prime}+1} \backslash \mathbf{V}_{k^{\prime}}^{t^{\prime}}$, then the path $w=u_{0}, \ldots, w^{\prime}=u_{l}$ built in the fourth loop satisfies $d_{\mathcal{K}}(\nu) \leq \varepsilon$ for all $\nu \in \bigcup_{0 \leq i<l}\left[\widehat{\delta_{u_{i}}}, \widehat{\delta_{u_{i+1}}}\right]$.

Proof. Let $\varepsilon>0$, there exists by compacity a $n_{\varepsilon} \in \mathbb{N}$ such that $f(\mu) \leq \frac{4}{n_{\varepsilon}} \Longrightarrow d_{\mathcal{K}}(\mu) \leq \varepsilon$. Iterating the same argument, there exists $k_{\varepsilon} \geq 3 n_{\varepsilon}$ such that $f(\mu) \leq \frac{4}{k_{\varepsilon}} \Longrightarrow d_{\mathcal{K}}(\mu) \leq b\left(n_{\varepsilon}\right)$.

Let $t_{\varepsilon}=\max _{0 \leq i \leq k_{\varepsilon}}\left(T_{i}\right)$ and assume $w \in \mathbf{V}_{k}^{t+1} \backslash \mathbf{V}_{k}^{t}$ and $w^{\prime} \in \mathbf{V}_{k^{\prime}}^{t^{\prime}+1} \backslash \mathbf{V}_{k^{\prime}}^{t^{\prime}}$ with $t^{\prime} \geq t \geq t_{\varepsilon}$. By definition of the $T_{i}$, we have $\mathbf{V}_{n}^{t_{\varepsilon}}=\mathbf{V}_{n}^{t}=\mathbf{V}_{n}^{t^{\prime}}$ for all $n \leq k_{\varepsilon}$. For $w$ and $w^{\prime}$ to be chosen by the 
algorithm, we must have $k \geq k_{\varepsilon}$ and $k^{\prime} \geq k_{\varepsilon}$ with $w \in \mathbf{V}_{k}$ and $w^{\prime} \in \mathbf{V}_{k^{\prime}}$ so $f\left(\widehat{\delta_{w}}\right)<\frac{3}{k_{\varepsilon}}<\frac{1}{n_{\varepsilon}}$ and $f\left(\widehat{\delta_{w^{\prime}}}\right)<\frac{3}{k_{\varepsilon}}<\frac{1}{n_{\varepsilon}}$, thus $w, w^{\prime} \in \mathbf{V}_{n_{\varepsilon}}$. Moreover, by definition of $k_{\varepsilon}, d_{\mathcal{K}}\left(\widehat{\delta_{w}}\right) \leq b\left(n_{\varepsilon}\right)$ and $d_{\mathcal{K}}\left(\widehat{\delta_{w^{\prime}}}\right) \leq b\left(n_{\varepsilon}\right)$.

Therefore $\bigcup_{u \in \mathbf{V}_{n_{\varepsilon}}} \mathbf{B}\left(\widehat{\delta_{u}}, b\left(n_{\varepsilon}\right)\right)$ contains $\widehat{\delta_{w}}$ and $\widehat{\delta_{w^{\prime}}}$ as well as $\mathcal{K}$ in a single connected component, since $\mathcal{K}$ is connected. This means that in the fourth loop of the algorithm, a path can be found for some $i \geq n_{\varepsilon}$. The path is entirely included in $\bigcup_{u \in \mathbf{V}_{i}} \mathbf{B}\left(\widehat{\delta_{u}}, b(i)\right)$. For $\nu \in \bigcup_{u \in \mathbf{V}_{i}} \mathbf{B}\left(\widehat{\delta_{u}}, b(i)\right)$, there exists $u \in \mathbf{V}_{i}$ such that $f(\nu) \leq f\left(\widehat{\delta_{u}}\right)+\frac{1}{i} \leq \frac{4}{i} \leq \frac{4}{n_{\varepsilon}}$ so $d_{\mathcal{K}}(\nu) \leq \varepsilon$ by definition of $n_{\varepsilon}$. The result follows.

$\diamond$ Claim 4

Claim 5: If $\mu \in \mathcal{V}\left(\left(w_{n}\right)_{n \in \mathbb{N}}\right)$, then $\mu \in \mathcal{K}$.

Proof. Take any $\varepsilon>0$, and wait that the first loop reaches the value $t=t_{\varepsilon}$ where $t_{\varepsilon}$ is defined in Claim 4. At some point, a new element $w_{n}$ will be found in the third loop and it will be added to the sequence already built (with a path of words before it). By construction, $w_{n} \in \mathbf{V}_{k}^{t}$ for some $t \geq t_{\varepsilon}$, and the same is true for any element found in the third loop from now on.

By Claim 4, this means that any pair of elements $\left(w_{k}, w_{k+1}\right)$ with $k \geq n$ added in the sequence from now on satisfies $\forall \nu \in\left[\widehat{\delta_{w_{k}}}, \widehat{\delta_{w_{k+1}}}\right], d_{\mathcal{K}}(\nu) \leq \varepsilon$. This is true for all $\varepsilon>0$, so any accumulation point of the polygonal path $\widehat{\bigcup_{n \geq N}\left[\widehat{\delta_{w_{n}}}, \widehat{\delta_{w_{n+1}}}\right]}$ is included in $\mathcal{K} . \quad \diamond$ claim 5

\section{Construction of a Cellular automaton Realising a given set OF MEASURES}

In this section, we prove a reciprocal to the computability obstructions of Proposition 3 and a partial reciprocal to Proposition 5 using Proposition 6. Given an uniformly computable sequence of words $\left(w_{n}\right)_{n \in \mathbb{N}}$ in $\mathcal{B}^{*}$, we construct a cellular automaton realising $\mathcal{V}\left(\left(w_{n}\right)_{n \in \mathbb{N}}\right)$ as its $\mu$-limit measures set. We remind that $\mathcal{V}\left(\left(w_{n}\right)_{n \in \mathbb{N}}\right)$ is defined as the set of limit points of the polygonal path defined by the sequence of measures $\left(\widehat{\delta_{w_{n}}}\right)_{n \in \mathbb{N}}$ :

$$
\mathcal{V}\left(\left(w_{n}\right)_{n \in \mathbb{N}}\right)=\bigcap_{N>0} \widehat{\bigcup_{n \geq N}\left[\widehat{\left[\delta_{w_{n}}, \widehat{\delta_{w_{n+1}}}\right]} .\right.}
$$

Theorem 1 (Realisation of a computable polygonal path of measures).

Let $\left(w_{n}\right)_{n \in \mathbb{N}}$ be a uniformly computable sequence of words of $\mathcal{B}^{*}$, where $\mathcal{B}$ is a finite alphabet. Then there is a finite alphabet $\mathcal{A} \supset \mathcal{B}$ and a cellular automaton $F: \mathcal{A}^{\mathbb{Z}} \rightarrow \mathcal{A}^{\mathbb{Z}}$ such that:

- for any measure $\mu \in \mathcal{M}_{\sigma-\text { mix }}^{\text {full }}\left(\mathcal{A}^{\mathbb{Z}}\right), \mathcal{V}(F, \mu)=\mathcal{V}\left(\left(w_{n}\right)_{n \in \mathbb{N}}\right)$.

- if $\mathcal{V}\left(\left(w_{n}\right)_{n \in \mathbb{N}}\right)=\{\nu\}$, then for any measure $\mu \in \mathcal{M}_{\sigma-\text { erg }}^{\text {full }}\left(\mathcal{A}^{\mathbb{Z}}\right), F_{*}^{t} \mu \underset{t \rightarrow \infty}{\longrightarrow} \nu$.

Furthermore we get an explicit bound for the convergence rate in the first point of the theorem. Assume that $w_{n}$ is computable in space $O(\sqrt{n})$ (by repeating elements of the sequence $\left(w_{n}\right)_{n \in \mathbb{N}}$ if necessary), one has:

$$
d_{\mathcal{M}}\left(F_{*}^{t} \mu, \mathcal{V}\left(\left(w_{n}\right)_{n \in \mathbb{N}}\right)\right)=O\left(\frac{1}{\log (t)}\right)+\sup \left\{d_{\mathcal{M}}\left(\nu, \mathcal{V}\left(\left(w_{n}\right)_{n \in \mathbb{N}}\right)\right): \nu \in \bigcup_{n \geq C(\log t)^{2}}\left[\widehat{\left[\delta_{w_{n}}\right.}, \widehat{\delta_{w_{n+1}}}\right]\right\}
$$

for some constant $C>0$. The first term of the upper bound corresponds to the intrinsic limitations of the construction, the second term depends on the speed of convergence of the polygonal path 
defined by $\widehat{\delta_{w_{n}}}$ towards $\mathcal{V}\left(\left(w_{n}\right)_{n \in \mathbb{N}}\right)$, which is intuitively the quality of the approximation of $\mathcal{V}(F, \mu)$ by a computable path.

This construction has many applications detailed in Section 5 . We just mention here Corollary 2 which says that every compact, $\Pi_{2}$-computable and connected subset of $\mathcal{M}_{\sigma}\left(\mathcal{B}^{\mathbb{Z}}\right)$ can be obtained as the $\mu$-limit measures set of a cellular automaton $F: \mathcal{A}^{\mathbb{Z}} \rightarrow \mathcal{A}^{\mathbb{Z}}$ for any $\mu \in \mathcal{M}_{\sigma-\text { mix }}^{\text {full }}\left(\mathcal{A}^{\mathbb{Z}}\right)$.

In the rest of this section, we detail the construction of this cellular automaton and prove the correctness of the construction in Subsection 3.5.

\subsection{Overview of the construction}

This section presents a sketch of the construction. The alphabet $\mathcal{A}$, where is defined the cellular automaton, contains a symbol $\mathrm{W}$ (for wall) persisting in time, except under special circumstances, defining independent areas of computation (segments). Independently in each segment, three tasks are performed in parallel:

Formatting: the initial contents of the segment are erased;

Computation and copy: each word $w_{i}$ is successively computed and concatenated copies of it are written on the whole segment;

Merging: the length of the segment is checked at regular intervals, and it merges with the segment to its right if it is too small.

The key task is the second, since the goal of the construction is that $F_{*}^{t} \mu$ gets close to each measure $\widehat{\delta_{w_{i}}}$ successively. This requires that the computation is performed synchronously between all segments, so that each segment contains copies of the same $w_{i}$ at the same instant. To do this, we define another symbol I (init), which appears only in the initial configuration, creating a wall and initialising computation and auxiliary processes. This process is detailed in Section 3.2.1.

Any symbol or process created in this way is referred to as initialised ; uninitialised processes are those already present in the initial configuration over which we have no control, and that we wish to erase. In particular, uninitialised walls are not considered as valid segment borders.

Apart from $\mathrm{I}$ and $\mathrm{W}$, the new alphabet $\mathcal{A}$ is divided in different layers: the main layer where the words $w_{n}$ are output and copied out, and auxiliary layers where computation and other processes take place. This allows to perform all tasks in parallel.

Formatting. Since we have no control over the initial contents of each segment, we first want to format the segment, that is, to erase uninitialised walls and uninitialised contents of the auxiliary layers.

Most processes defined below are designed to self-destruct when they are not initialised. This is detailed as each new process is introduced. The difficult task is to distinguish uninitialised walls from initialised walls.

To do that, each initialised wall sends to its right a signal on a specific layer progressing at speed one (formatting counter - see Section 3.2.4), that keeps track of its age using a binary counter. Meanwhile, each initialised walls also keeps track of its age under the form of a binary counter on another layer, to its left, incrementing at each step (time counter - see Section 3.2.3).

Time and formatting counters already present in the initial configuration (uninitialised) have a nonnegative value at time 0 , whereas those created by an I symbol (initialised) have value 0 at time 1, and they increment at the same rate. Thus, uninitialised walls have older time counters, and by comparing time counters and formatting counters as they cross, we can erase older counters and uninitialised walls. Figure 1 is an overview of those processes.

Computation and copy. Meanwhile, on another layer, a Turing machine is simulated in the space delimited by the time counter. This machine successively computes each $w_{n}$ (see Section 3.3.2) and 


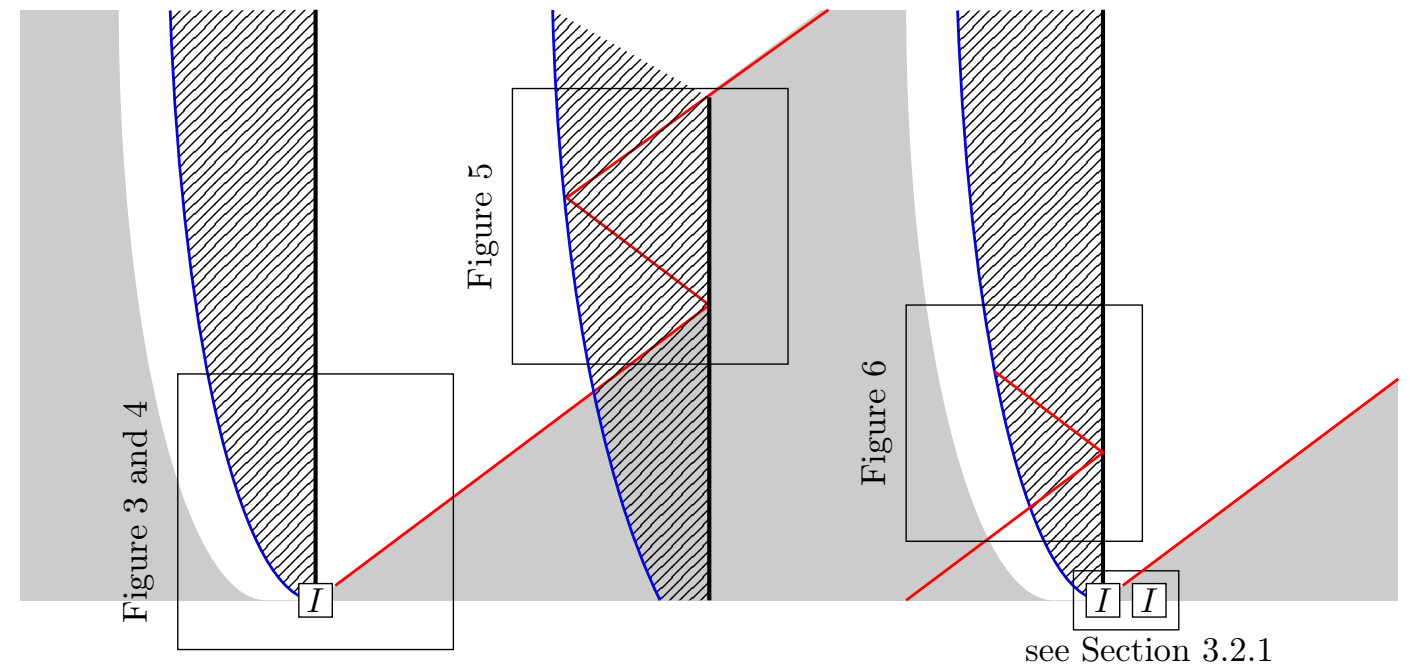

FigURE 1. Sketch of the bootstrapping and formatting processes. Vertical lines are walls. Dashed parts contain time counters (section 3.2.3) and Turing machines (section 3.3.2). Slanted lines are formatting counters (section 3.2.4), white and grey areas are respectively formatted and non-formatted.

writes concatenated copies on the main layer of the segment to its left (see Section 3.3.3). For each $w_{n}$, this happens synchronously on the whole configuration, so as to approach the measure $\widehat{\delta_{w_{n}}}$.

Merging. Synchronously at some time $T_{n}$, segments of a given length $n$ are merged with their left neighbour. This allows us to enlarge computational space and decrease the density of cells with nonempty auxiliary layers, so that they do not appear in the limit measure (see Section 3.4). To determine the length of its right segment, each wall sends a signal to the right on a dedicated layer that bounces off the next wall and counts the return time. Figure 2 is an overview of copy and merging processes.

Alphabet. We obtain an enlarged alphabet $\mathcal{A}=\{\mathrm{I}, \mathrm{W}\} \cup \mathcal{A}_{\text {main }} \times \mathcal{A}_{\text {comp }} \times \mathcal{A}_{\text {time }} \times \mathcal{A}_{\text {format }} \times \mathcal{A}_{\text {copy }} \times$ $\mathcal{A}_{\text {merge }}$. All those alphabets contain a symbol \# (blank) representing the absence of information.

- I and $\mathrm{W}$ are the two above-mentioned symbols;

- $\mathcal{A}_{\text {main }}=\mathcal{B} \cup\{\#\}$ is the layer on which $w_{n}$ is output and then copied out;

- $\mathcal{A}_{\text {comp }}$ is the layer where Turing machines are simulated to compute $w_{n}$ and other processes;

- $\mathcal{A}_{\text {time }}$ is the layer on which time counters are incremented;

- $\mathcal{A}_{\text {format }}$ is the layer on which formatting counters move and are incremented, and where comparisons are done;

- $\mathcal{A}_{\text {copy }}$ is a layer used in the process of writing copies of the output on the main layer;

- $\mathcal{A}_{\text {merge }}$ is a layer used in the process of merging two segments.

We have $\mathcal{B} \subset \mathcal{A}$ up to the identification $b \Longleftrightarrow(b, \#, \#, \#$, \#, \#). If $u \in \mathcal{A}$, denote main $(u)$, resp. $\operatorname{comp}(u)$, time $(u) \ldots$ the projections on each layer (the result being \# on $\mathrm{I}$ and $\mathrm{W}$ ).

We detail the different alphabets in the following sections. As we will see, our construction needs interactions at a distance at most three, so we can take $\mathbb{U}_{F}=\{-3, \ldots, 3\}$ as the neighbourhood of the local rule of $F$. 


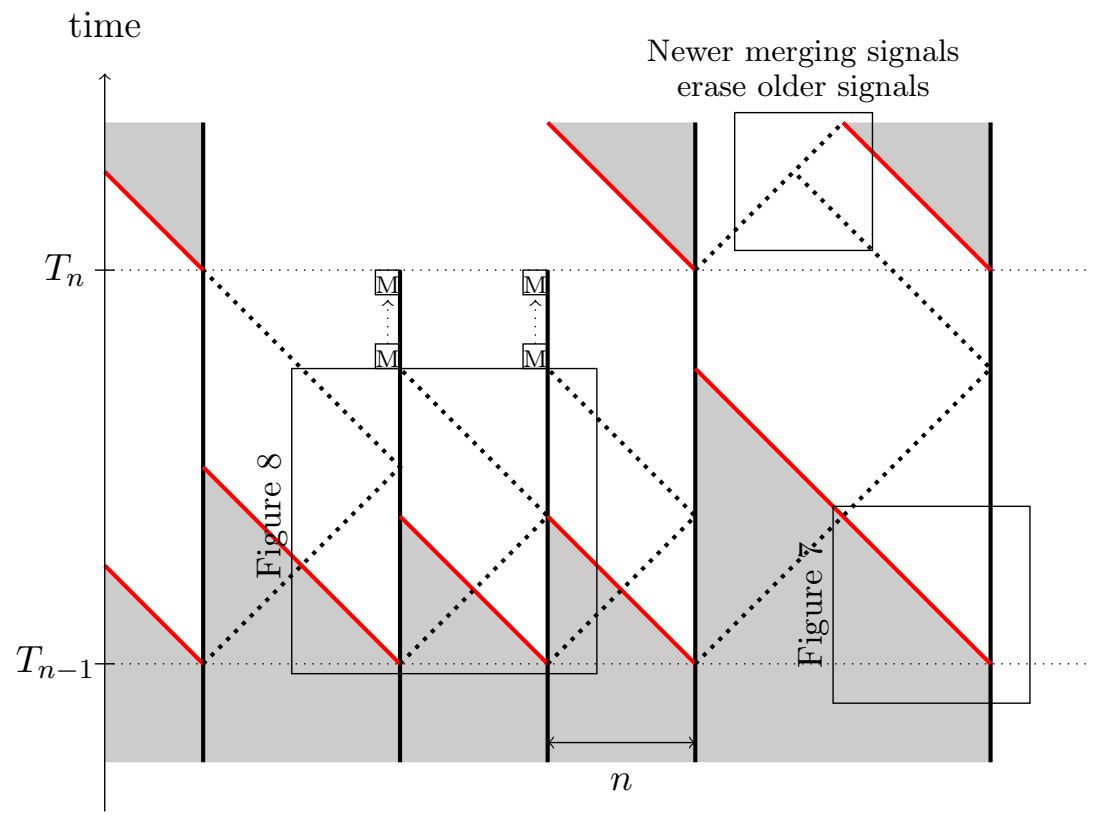

FIgURE 2. Sketch of the copying and merging processes. Here all walls are initialised. Slanted thick lines are copy processes (see Section 3.3.3), slanted dotted lines are merging signals (see Section 3.4).

\subsection{Formatting the segments}

\subsubsection{Bootstrapping}

If two symbols I are separated by two cells or less, the rightmost one is destroyed. Otherwise, every I symbol turns into a $\mathrm{W}$, erases the contents of three cells to their right and left (including walls), and initialises on its left a computation process and a time counter, and on its right a formatting counter. No more $\mathrm{I}$ or $\mathrm{W}$ symbols can be created.

Definition 9. Let $x \in \mathcal{A}^{\mathbb{Z}}$. The set of positions $[i, j]$ is a segment at time $\mathbf{0}$ if $x_{i}$ and $x_{j}$ are the symbol $\mathrm{I}$ and this symbol does not appear for intermediate coordonate in $] i, j[$. It is a segment at time $t$ if $F^{t}(x)_{i}$ and $F^{t}(x)_{j}$ are initialized walls $\mathrm{W}$ (that is to say $x_{i}=x_{j}=\mathrm{I}$ ) and there is not initialized walls between them at time $t$. Define the length of this segment as $j-i-1$.

Walls persist over time and are only destroyed under three circumstances:

- if the time layer of the computation layer of the cell to its left is empty (so the wall must be uninitialised);

- by a growing time counter (see Section 3.2 .3 and Facts 2 , 6 and 7);

- by the merging process detailed in Section 3.4.

As the only exception, if a segment is of length three at time 0 , then the leftmost I prevents the creation of a time counter for the rightmost wall at time 1 and the wall itself is destroyed at time 2. Thus segments have minimum length four from time 2 onwards.

\subsubsection{Counters}

All counters are binary in a redundant basis, so that they can be incremented by one at each step (keeping track of current time) in a local manner. Notice that in the following two definitions, the indexing of the letters is inverted. 
Definition 10 (Redundant binary basis). Let $u=u_{0} \ldots u_{n-1} \in\{0,1,2\}^{*}$. The value of $u$ is

$$
\operatorname{val}(u)=\sum_{i=0}^{n-1} u_{i} 2^{i}
$$

Since the basis is redundant, different words in $\{0,1,2\}^{*}$ can have the same value.

Definition 11 (Incrementation). The incrementation operation inc : $\{0,1,2\}^{*} \longmapsto\{0,1,2\}^{*}$ is defined in the following way. If $u_{|u|-1}=2$, then $|\operatorname{inc}(u)|=|u|+1,|u|$ otherwise, and:

$$
\operatorname{inc}(u)_{i}=\left\{\begin{array}{lll}
1 & & \text { if } i=|u| \text { and } u_{|u|-1}=2 \\
u_{i} & \bmod 2+1 & \text { if } i=0 \text { or } u_{i-1}=2 \\
u_{i} & \bmod 2 & \text { otherwise }
\end{array}\right.
$$

Intuitively, the counter is increased by one at the rightmost bit and 2 behaves as a carry propagating along the counter. If the most significant bit was a carry, the length of the counter is increased by one. Thus:

Fact 1. $\operatorname{val}(\operatorname{inc}(u))=\operatorname{val}(u)+1$.

Taking a symbol as spark where the counter is incremented, in our case the symbol $\mathrm{W}$, and another one to precise the end of the word, in our case \#, this operation can be defined locally and can be seen as the local rule of a cellular automaton.

\subsubsection{Time}

We use the alphabet $\mathcal{A}_{\text {time }}=\{0,1,2$,\# . In a configuration, a time counter is a word of maximal length containing no \# in the time layer. A time counter is attached if it is bounded on its right

\begin{tabular}{|c|c|c|c|c|c|c|c|c|c|c|}
\hline$\#$ & $\#$ & \# & $\#$ & $\#$ & \# & \# & 1 & 0 & 2 & $\mathrm{~W}$ \\
\hline \# & $\#$ & \# & $\#$ & $\#$ & \# & 7 & $\#$ & 2 & 1 & $\mathrm{~W}$ \\
\hline$\#$ & $\#$ & $\#$ & $\#$ & $\#$ & & & $\#$ & 1 & 2 & $\mathrm{~W}$ \\
\hline$\#$ & 1 & $\#$ & $\#$ & $\#$ & & & $\#$ & 1 & 1 & $\mathrm{~W}$ \\
\hline$\#$ & 1 & 0 & $\#$ & $\#$ & $\#$ & $\#$ & $\#$ & $\#$ & 2 & $\mathrm{~W}$ \\
\hline \# & 1 & 0 & 0 & $\#$ & & $\#$ & $\#$ & 7 & 1 & $\mathrm{~W}$ \\
\hline \# & $\#$ & 2 & 0 & 0 & & $?$ & $\#$ & $\neq$ & 0 & $\mathrm{~W}$ \\
\hline$?$ & $\#$ & 1 & 2 & 0 & 1 & \# & $?$ & $?$ & $?$ & I \\
\hline
\end{tabular}
by a wall $\mathrm{W}$, detached otherwise.

FiguRE 3. A detached time counter, and a time counter attached to an initialised wall. Only the time layer is represented. ? cells have arbitrary values.

At each step, attached counters are incremented by one while detached counters have their rightmost bit deleted (see Figure 31). Indeed, detached counters are uninitialised and can be safely deleted. Formally,

- if $u_{1}=\mathrm{W}$, then time $\left(F(u)_{0}\right)=\operatorname{time}\left(u_{0}\right) \bmod 2+1$;

- if $\operatorname{time}\left(u_{1}\right)=\#$, then $\operatorname{time}\left(F(u)_{0}\right)=\#$; 
- otherwise, follow the incrementation definition (Definition 11).

When a counter increases in length, it may erase a wall by overwriting it. However, this is not a problem, as we shall see in Facts 2 and 6.

Fact 2. An initialised wall cannot be erased by a detached time counter.

Proof. A detached time counter is not incremented and can extend by one cell at most because of the carries initially present in the word. But I symbols erase two cells to their right at initialisation.

Fact 3. Let $x \in \mathcal{A}^{\mathbb{Z}}$ be the initial configuration. Each attached time counter $u$ in $F^{t}(x)$ satisfies $\operatorname{val}(u) \geq t-1$, the equality being attained if this counter is attached to an initialised wall.

Proof. No time counter is created except at $t=1$ (by $\mathrm{I}$ ). Therefore such a counter was present either in the initial configuration (with a nonnegative value), or was created at $t=1$ by a $\mathrm{I}$ symbol. It is incremented by one at each step in both cases.

Thus we can use time counters to tell apart initialised walls from non-initialised walls, which is the object of the next section.

\subsubsection{Formatting and comparisons}

We want to implement a counter in a new layer which is compared to the time counter when they are in interaction. The formatting layer $\mathcal{A}_{\text {format }}$, contains the symbol \# as all layers. A formatting counter is a word of maximal length where the value of the cell in $\mathcal{A}_{\text {format }}$ is different than \#. Formatting counters are defined and incremented at each step in a similar way as time counters, but they have a range of different behaviours. Thus the other elements of $\mathcal{A}_{\text {format }}$ are decomposed into two layers $\mathcal{A}_{\text {value }}=\{0,1,2, \#\}$ and $\mathcal{A}_{\text {state }}$ where the possible value are:

"Go" state: The counter progresses at speed one to the right.

"Stop" state: Once a wall is encountered, the counter progressively (right to left) stops.

Comparison states: Once the whole counter has stopped, we locally compare the formatting counter and the time counter, left to right, with a method we describe later which use the symbol $\left\{-,=_{-},=,=_{+},+\right\}$.

The wall is destroyed if the formatting counter is strictly younger, and the formatting counter is destroyed otherwise (see Figures 5 and 6). In the former case, the counter progressively returns to the "Go" state.

Changing state takes some time to propagate the information along the counter. Therefore, counters passing from a "Go" state to a "Stop" state are temporarily in a situation where the left part of the counter progresses whereas the right part has not. To avoid erasing information, counters in a "Go" state have buffers, i.e. the value of the counter is only written on half the cells, the other half containing (Go,\#) (see Figure 44).

When its length increase, a counter never merges with another counter, erasing bits from the right-hand counter instead in order to avoid merging: we say the right-hand counter is dominated. Notice that it is impossible for a counter located to the right of another counter to be initialised, and so it is safe to erase bits of it.

Fact 4. Let $x \in \mathcal{A}^{\mathbb{Z}}$ be the initial configuration. Any non-dominated formatting counter $u$ of $F^{t}(x)$ satisfies $\operatorname{val}(u) \geq t-1$, the equality being attained if the counter is initialised.

Proof. Similar to Fact 3 . 


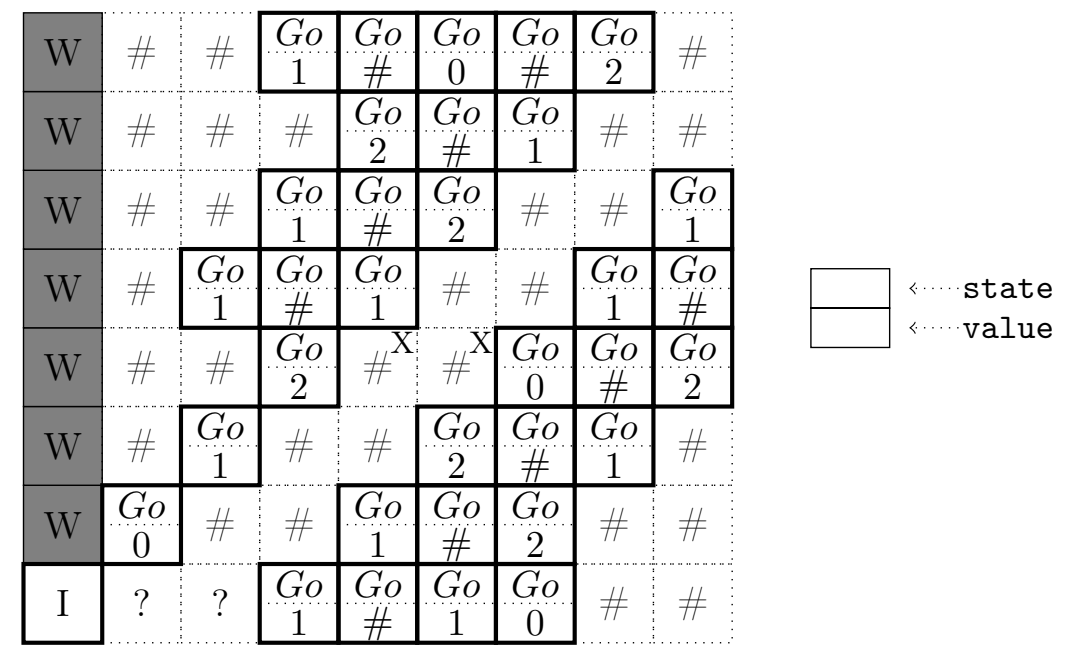

Figure 4. One initialised and one uninitialised formatting counter. X symbols mark the cells where values are prevented to appear to avoid merging: the right counter is dominated. Only the formatting layer is represented.

Thus we guarantee that an initialised (hence non-dominated) formatting counter is strictly younger than any uninitialised wall, and symmetrically. Uninitialised formatting counters can only progress to the right to be destroyed by the nearest initialised wall. We will see that dominated counters, whose value is arbitrary, are not a problem since they are erased before any comparison takes place.

Definition 12 (Comparison method). Let $u=u_{0} u_{1} \ldots$ and $v=v_{0} v_{1} \ldots$ be two counters in redundant binary basis (adding zeroes so that $|u|=|v|$ ). Let us note $\operatorname{sign}(u-v)$ the result of the comparison between $u$ and $v$, that is,,+ 0 or - .

Case 1: if $|u|=|v|=1, \operatorname{sign}(u-v)=\operatorname{sign}\left(u_{0}-v_{0}\right)$;

Case 2: if $u_{0}+\left\lfloor u_{1} / 2\right\rfloor>v_{0}+\left\lfloor v_{1} / 2\right\rfloor+1$, then $\operatorname{sign}(u-v)=+$, and symmetrically;

Case 3: if $u_{0}+\left\lfloor u_{1} / 2\right\rfloor=v_{0}+\left\lfloor v_{1} / 2\right\rfloor+\varepsilon$ (for some $\left.\varepsilon \in\{-1,0,1\}\right)$, then $\operatorname{sign}(u-v)=$ $\operatorname{sign}\left(\left(u_{1}^{\prime}+2 \varepsilon\right) u_{2} \cdots-v_{1}^{\prime} v_{2} \ldots\right)$,

where $u_{1}^{\prime}=u_{1} \bmod 2$ and $v_{1}^{\prime}=v_{1} \bmod 2$.

In other words, we do a bit-by-bit comparison starting from the most significant bit, considering that \# is equal to 0 , and taking into account the carry propagation "in advance", so that the incrementation and carry propagation can continue during the comparison. When the "local difference" $\varepsilon$ is too small, the result cannot be determined locally and a remainder is carried (consider a comparison between $120 \cdots 0$ and $11 \cdots 12)$.

Formally, for each pair of bits $\left(u_{n}, v_{n}\right)$, we add 1 to each bit if the following bit of the corresponding counter is 2 , and depending on the value of $u_{n}-v_{n}+2 \varepsilon$ :

\begin{tabular}{|c|c|c|c|c|c|}
\hline result & $<-1$ & -1 & 0 & +1 & $>+1$ \\
\hline new state & - & $=_{-}$ & $=$ & $=_{+}$ & + \\
\hline
\end{tabular}

If the result can be determined locally (cases 1 and 2), the state is changed to + or - , and the result propagates to the right without further comparisons. Otherwise (case 3), the state changes to $=$, which means future bit comparisons will decide the result in the same way. If there is a remainder $\varepsilon$, it is remembered for the next comparison by having three states $=_{-},=_{+},=$. See Figure 6 for an example. 


\begin{tabular}{|c|c|c|c|c|c|c|c|}
\hline$\#$ & $\#$ & $\#$ & $\#$ & $=$ & Go & Go & Go \\
\hline$\#$ & $\#$ & $\#$ & $\#$ & $=$ & - & Go & $\mathrm{W}$ \\
\hline$\#$ & $\#$ & $\#$ & $\#$ & $=$ & - & - & $\mathrm{W}$ \\
\hline$\#$ & $\#$ & $\#$ & $\#$ & $=$ & - & Stop & $\mathrm{W}$ \\
\hline$\#$ & $\#$ & $\#$ & $\#$ & $=$ & Stop & Stop & $\mathrm{W}$ \\
\hline$\#$ & $\#$ & $\#$ & $\#$ & Stop & Stop & Stop & $\mathrm{W}$ \\
\hline$\#$ & $\#$ & $\#$ & Go & Go & Stop & Stop & $\mathrm{W}$ \\
\hline$\#$ & $\#$ & Go & Go & Go & Go & Stop & $\mathrm{W}$ \\
\hline$\#$ & Go & Go & Go & Go & Go & \# & $\mathrm{W}$ \\
\hline
\end{tabular}

FiguRE 5. A younger formatting counter encountering an older wall, which is destroyed. Only the state layer of $\mathcal{A}_{\text {format }}$ is represented, with greyed words for buffers.

\begin{tabular}{|c|c|c|c|c|c|c|}
\hline \multirow[t]{2}{*}{$\#$} & $\overline{=}$ & $\overline{=}$ & $=+$ & \multirow{2}{*}{ \# } & \multirow[t]{2}{*}{ \# } & \multirow[t]{2}{*}{ W } \\
\hline & $11: 1$ & 000 & 100 & & & \\
\hline \multirow{2}{*}{ \# } & $=$ & $=$ & $=+$ & + & \multirow{2}{*}{$\#$} & \\
\hline & $1: 1$ & $0: 0$ & 10 & $1 / 1$ & & \\
\hline \multirow{2}{*}{$\#$} & $=$ & $=$ & $=_{+}$ & + & + & \multirow{2}{*}{ W } \\
\hline & $1 \#$ & $\begin{array}{lll}0 & 2\end{array}$ & 10 & $\begin{array}{lll}0 & 1\end{array}$ & $\begin{array}{l:l}2 & 1\end{array}$ & \\
\hline \multirow{2}{*}{$\#$} & $=$ & $=$ & $={ }_{+}$ & + & Stop & \multirow[b]{2}{*}{$y$} \\
\hline & $1: \#$ & $0: 1$ & $0: 2$ & $2: 0$ & $1: 2$ & \\
\hline \multirow{2}{*}{$\#$} & \multirow{2}{*}{ \# } & $=$ & $=+$ & Stop & Stop & \multirow{2}{*}{ W } \\
\hline & & $2: 1$ & $0: 1$ & 122 & $2: 1$ & \\
\hline \multirow{2}{*}{ \# } & \multirow{2}{*}{ \# } & $=$ & Stop & Stop & Stop & \multirow[b]{2}{*}{ W } \\
\hline & & $1: 1$ & 21 & $\begin{array}{lll}1 & 1\end{array}$ & 12 & \\
\hline \multirow{2}{*}{ \# } & \multirow{2}{*}{ \# } & Stop & Stop & Stop & Stop & \multirow{2}{*}{ W } \\
\hline & & $1: 1$ & $\begin{array}{l:l}1 & 1\end{array}$ & $\begin{array}{lll}2 & 1 \\
\end{array}$ & $2 \vdots 1$ & \\
\hline
\end{tabular}

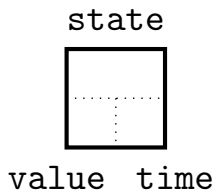

FiguRE 6. The comparison process in detail. Here the formatting counter is older than the time counter and is destroyed. Only the layers $\mathcal{A}_{\text {time }}$ and $\mathcal{A}_{\text {format }}$ are represented.

After the comparison, two cases are possible:

- if the state of the rightmost bit is - or $=_{-}$, the wall is strictly older than the counter. The wall is destroyed and the state of the rightmost bit becomes "Go". The counter then progressively returns to the "Go" state.

- if the state of the rightmost bit is,$+=_{+}$or $=$, the wall is younger than the counter. The rightmost bit is erased, and the rest of the counter is progressively erased in a similar way as a detached time counter. 
The second case covers the case where both the counter and the wall are initialised (result =), which means that the formatting counter has finished formatting its segment and may be erased. Also, if the counter is dominated, then its leftmost bit is erased at each step, preventing the comparison to start, until the counter is entirely erased.

To sum up, $\mathcal{A}_{\text {format }}=\{\#\} \cup(\{G o\} \times\{0,1,2, \#\}) \cup\left(\left\{S t o p,+,-,=,=_{+},=_{-}\right\} \times\{0,1,2\}\right)$.

When a formatting counter reaches the right wall of the segment, the segment is said to be formatted. This implies that the segment contains no more uninitialised walls.

Fact 5. At time $k(1+\lceil\log k\rceil)$, all segments of length $k$ (for $k>3$ ) are formatted.

Proof. As long as $t \leq k(1+\lceil\log k\rceil)$, any initialised formatting counter has length $\lceil\log t\rceil \leq 2\lceil\log k\rceil$ (excluding the buffers) since it is in base 2. The counter progresses at speed one except when it meets another wall. Each comparison takes a time equal to twice the current length of the counter (again excluding the buffers). Furthermore, two consecutive walls are separated by three cells at least (cf. Section 3.2.1). Thus, the segment is formatted in less than $k+\frac{k}{4} \cdot 2 \cdot 2\lceil\log k\rceil$ steps, which is coherent with our first assumption.

Fact 6. An initialised wall cannot be erased by a time counter attached to a uninitialised wall.

Proof. Consider two walls, the left being initialised and the right uninitialised. As explained in Section 3.2.1, we can assume they are separated by $k>3$ cells. The value of the time counter attached to the right wall cannot exceed $2^{k-3}$ at time 1 (since every symbol $\mathrm{I}$ erases three cells to its right at time 1), so it takes more than $2^{k}-2^{k-3}$ steps before the left wall is erased. According to Fact 5, the right wall is destroyed in less than $k(1+\lceil\log k\rceil)$ steps, and from then its time counter takes at most $k$ more steps to be erased.

For $k \geq 5, k(1+\log k)+k \leq 2^{k}-2^{k-3}$, so the counter is erased before it reaches the left wall. For $k=4$, any wall between them is destroyed at time 1 , so the destruction time is actually less than $k+2 \log k+k \leq 2^{k}-2^{k-3}$.

\subsection{Computation and copy}

\subsubsection{Simulating a Turing machine in a cellular automaton}

Let $\mathcal{T} \mathcal{M}=\left(Q, \Gamma, \#, q_{0}, \delta, Q_{F}\right)$ be a Turing machine. We simulate this machine in a cellular automaton $F$ on the alphabet $(\Gamma \cup \#) \times(Q \cup \#)$. The left part contains the content of the tape; the right part contains the state of the machine for the cell where the head is located, and \# everywhere else.

The local rule of $F$ is governed by the rules of the machine. That is, for all $u \in((\Gamma \cup \#) \times(Q \cup \#))^{\mathbb{Z}}$, and writing _ to denote an arbitrary value:

- if the head is on $u_{0}$ and $\delta\left(u_{0}\right)=\left(q, \gamma,{ }_{-}\right)$, then $F(u)_{0}=(\gamma, \#)$;

- if the head is on $u_{1}, \delta\left(u_{1}\right)=(q, \ldots, \leftarrow)$ and $u_{0}=\left(\gamma^{\prime}, \#\right)$, then $F(u)_{0}=\left(\gamma^{\prime}, q\right)$;

- similarly if the head is on $u_{-1}$ and $\delta\left(u_{-1}\right)=\left(q,{ }_{-}, \rightarrow\right)$;

- otherwise, $F(u)_{0}=u_{0}$.

When starting from a configuration filled with (\#,\#) everywhere except for a finite window with only one head, the time evolution of the cellular automaton matches the time evolution the Turing machine. The Turing machine considered in the proof does not stop, but by consistency we can assume that when the machine has stopped (the state being in $Q_{F}$ ), the local rule is the identity function. 


\subsubsection{Computation}

Computation takes place to the left of each initialised wall. $\mathcal{A}_{\text {comp }}$ is divided into three layers, on which three Turing machines are simulated, using the alphabet

$$
\mathcal{A}_{\text {comp }}=\bigotimes_{i=1}^{3}\left(\Gamma_{i} \cup \#\right) \times\left(Q_{i} \cup \#\right) \text {. }
$$

We adapt the simulation so that these Turing machines can read input from or write output to another layer (when indicated).

We now describe the operations to be performed symchronously between times $T_{n-1}$ and $T_{n}$ that we will fix later. Assume that, at time $T_{n-1}, n$ is already written on the layer 1 and $T_{n-1}$ on layer 3. The machines:

(1) replace $n$ by $n+1$ on layer 1 and stops;

(2) compute $w_{n}$ on layer 2, outputting it on the main layer, and stops;

(3) compute $T_{n}$ on layer 3 , and stops;

When $t=T_{n}$ ( $t$ being read from the time layer), the copying process triggers and the next computation starts, except when merging occurs; see next subsections.

All these operations must be performed in less than $T_{n}-T_{n-1}$ steps. We now fix the value of $T_{n}$ so that it is indeed possible.

A Turing machine with tape alphabet $\Gamma$ and set of states $Q$ and using only a computational space $S$ stops in time $S \cdot|\Gamma|^{S} \cdot|Q|$ which is the number of possible configurations. Otherwise, the same configuration would be reached twice, entering a loop.

Therefore there exists a constant $q>0$ large enough that the operations on layers 1 and 2 can be performed in space $\lfloor\sqrt{n}\rfloor \log _{2} q$ and time $O\left(q^{\lfloor\sqrt{n}\rfloor}\right)$. Furthermore, the function $(r, n) \longmapsto r^{\lfloor\sqrt{n}\rfloor}$ is computable in space $\lfloor\sqrt{n}\rfloor \log _{2} r$ (length of the output) and time $O\left(n^{3 / 2}(\log r)^{2}\right)$ (compute $\lfloor\sqrt{n}\rfloor$ in time $O(n)$, then perform $\lfloor\sqrt{n}\rfloor$ multiplications between numbers of length $\lfloor\sqrt{n}\rfloor \log _{2} r$ at most in time $\left.O\left(\left(\sqrt{n} \log _{2} r\right)^{2}\right)\right)$.

In other words, if we fix

$$
T_{n}-T_{n-1}=q^{\lfloor\sqrt{n}\rfloor},
$$

then the operation on layer 3 can be performed in space $\lfloor\sqrt{n}\rfloor \log _{2} q$ and time $O\left(q^{\lfloor\sqrt{n}\rfloor}\right)$. However, we need an upper bound on the time at each step and not only an asymptotic bound. This is solved by the linear speedup theorem for Turing machines: we can divide the computational time by any fixed constant $C$ by replacing each machine $M_{i}$ by a new machine $M_{i}^{\prime}$, such that $M_{i}^{\prime}$ performs $C$ computational steps of $M_{i}$ at each step, increasing the radius as necessary.

Remark. We fixed $T_{n}$ so that the computation space is of size $\sqrt{n}$ at time $T_{n}$ and constitutes an asymptotically negligible fraction of its segment. We could choose instead of $\sqrt{n}$ any other function in $o(n)$ which is time constructible.

Similarly to time counters, whenever they find an empty computational layer to their right (instead of a wall or another computation state), computation states was replaced by the symbol $(\#, \#)$. Thus uninitialised computation states self-destruct progressively. This requires that the Turing machines are adapted so that they never write (\#,\#) in a cell in the middle of a computation.

\subsubsection{Copying}

On the layer $\mathcal{A}_{\text {copy }}$, the cellular automaton copies periodically the words produced by the Turing machine in view to make samplings of the limit measures, we just put $\mathcal{A}_{\text {copy }}=\mathcal{B} \cup\{\#\}$.

At time $T_{n}(n \geq 0), w_{n}$ has been output on the main layer, followed by a symbol \#. If the segment is not in the process of merging, repeated copies of $w_{n}$ have to be written over the main 
layer. The Turing machine triggers the copying process by copying the rightmost letter of $w_{n}$ from the main layer to the copy layer.

First phase: As long as it has not met a symbol \#, the word on the copy layer progresses at speed -2 (that is to say if it is in the position $[i, j]$ it moves to the position $[i-2, j-2]$ ) and at each step a letter is copied from the main layer to the tail of the word;

Second phase: The word keeps progressing at speed -2 but the head loses one letter at each step and copies it on the main layer. The tail keeps copying letters from the main layer.

Intuitively, the cellular automaton performs a caterpillar-like movement between the copy and main layers (see Figure 7 for an example). The process ends when it meets a wall.

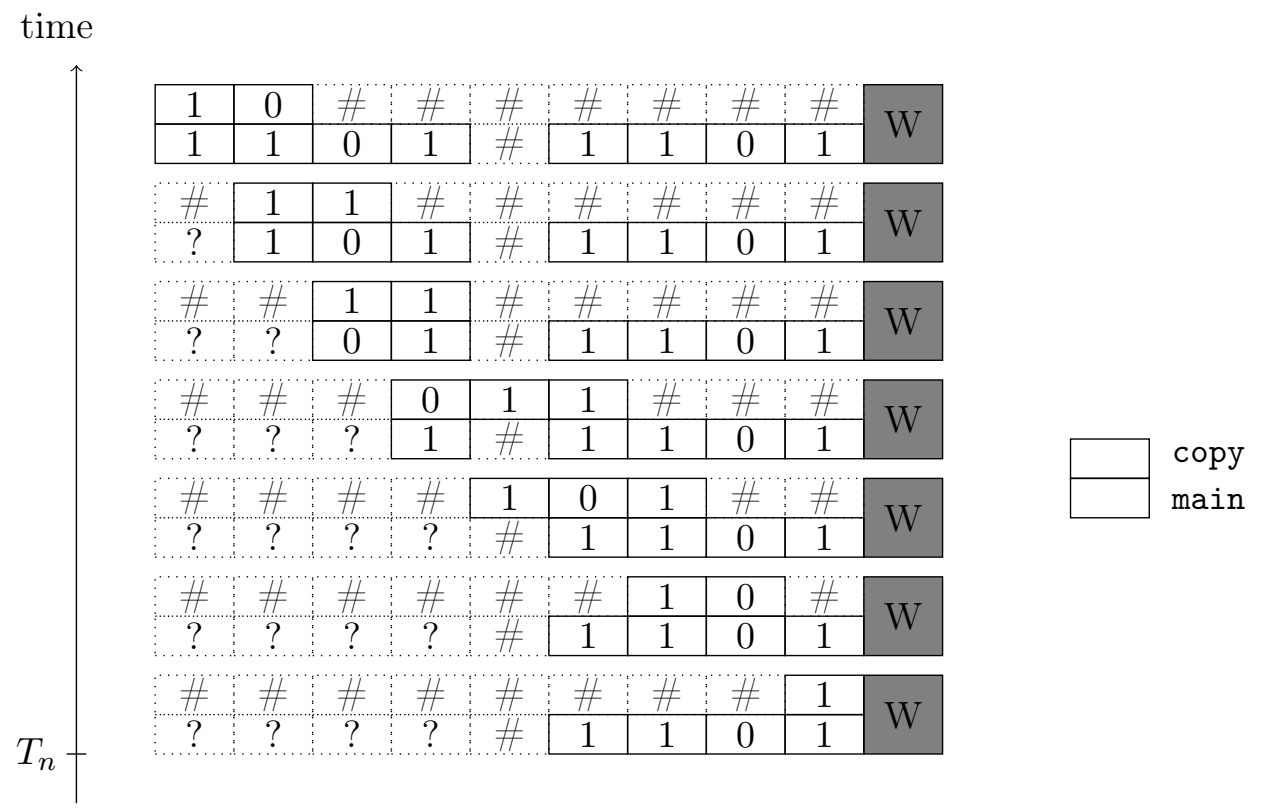

Figure 7 . Beginning of the copying process, with $w_{n}=1101$. Only the layers $\mathcal{A}_{\text {copy }}$ and $\mathcal{A}_{\text {main }}$ are represented.

Uninitialised copying processes may write arbitrary words on the main layer, but they progress to the left at speed one and are destroyed by the nearest wall in this direction.

\subsection{Merging}

At time $T_{n}$, all segments of length $n$ are forced to merge with their left neighbour, so that the density of walls tends to 0 . This means that merging is performed at time $T_{n}$ between a segment larger than $n$ to the left, and any number of consecutive segments of length $n$ to the right. To determine the length of each segment, a signal is sent to the right and bounces off the right wall, and its return time is measured.

To do so, a merging counter of value $2 n$ is initialised at time $T_{n-1}$ on the merge layer. The value of $n$ is copied from the first computing layer to the merge layer (with an additional 0 at the end), using an auxiliary state $\mathrm{C}$ (copy). This counter decrements at each step in a similar way as incrementing counters, except it uses -1 as a negative carry. See Figure 8 for an example of this process. 
If the signal returns at or before the end of the decrementation, a symbol $\mathrm{M}$ (merge) is created on the merge layer to indicate that the wall is to be destroyed at the next $T_{n}$; this is the only case where the copying process described above does not trigger. To sum up,

$$
\mathcal{A}_{\text {merge }}=\{-1,0,1, \mathrm{M}, \mathrm{C}\} \times\{\rightarrow, \leftarrow\} \cup\{\#\} .
$$

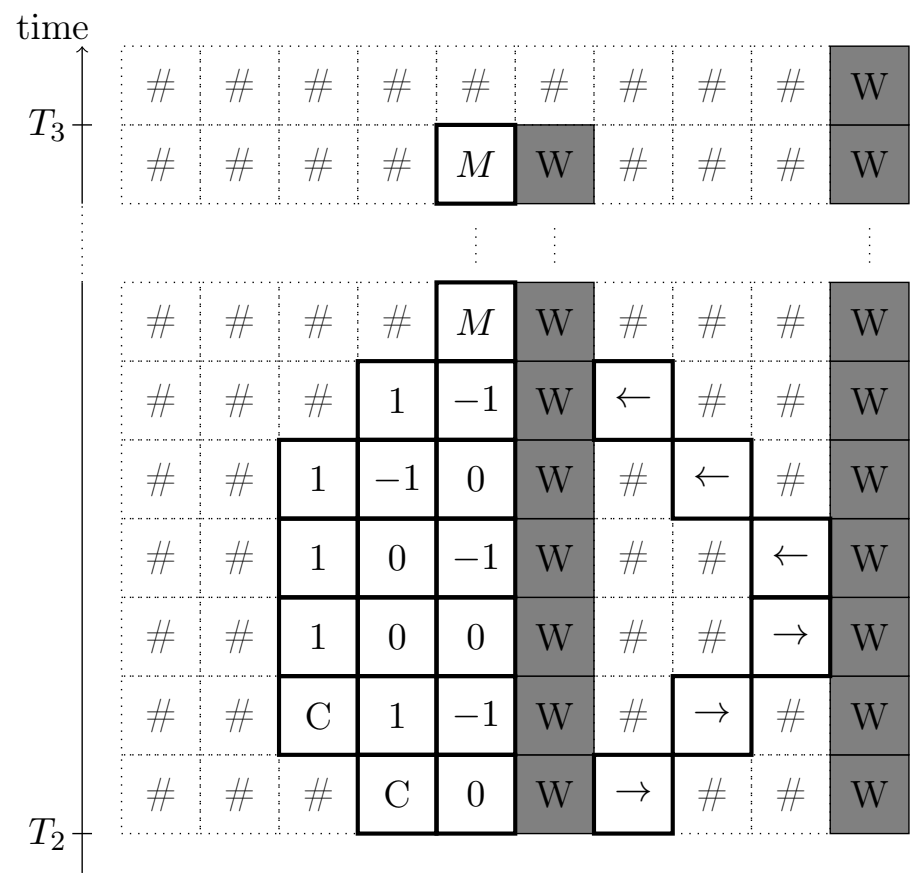

Figure 8. Determination of the length of a segment. Here the right segment is of length 3 and the two segments merge at time $T_{3}$. Only the merging layer is represented, with the counter of the right segment omitted for clarity.

Fact 7. Left walls of segments of length $\ell$ are erased at time $T_{\ell}^{\prime}=\min \left(T_{\ell}, 2^{\ell}+\ell\right)$.

Proof. Except for the situation described above, the only other way for an initialised wall to be erased is a time counter attached to an initialised wall, see Facts 2 and 6. A redundant binary counter whose initial value is 0 reaches length $\ell$ at time $2^{\ell}+\ell$, the second term come from the carry propagation.

Uninitialised merging counters are destroyed in exactly the same way as uninitialised time counters. To prevent uninitialised merging signals from disturbing a merging process, any right merging signal $\rightarrow$ erase incoming left merging signals $\leftarrow$. Merging signals arriving to a wall outside of a merging process is simply ignored and destroyed.

\subsection{Correctness of the construction}

To sum up, we have two time sequences $\left(T_{n}\right)_{n \in \mathbb{N}}$ and $\left(T_{n}^{\prime}\right)_{n \in \mathbb{N}}$ such that:

- At time $T_{n}$, the computation of $w_{n}$ is finished and the copy starts;

- At time $T_{n}^{\prime}$, the segments of length $n$ merge with their left neighbour.

Furthermore, those sequences are equal for $n$ large enough. 
The computation, copy and merging processes described in the previous section have to be performed between time $T_{n}$ and time $T_{n+1}$, which requires that the segments are not too large. In this section, we control the length of segments at time $T_{n}$.

Proposition 7. $T_{n}=\Theta\left(\lfloor\sqrt{n}\rfloor q^{\lfloor\sqrt{n}\rfloor}\right)$ where $q$ is defined in Section 3.3.2.

Proof. $T_{n}=\sum_{k=1}^{n} T_{k}-T_{k-1}$. Since $T_{k+1}-T_{k}=q^{\lfloor\sqrt{k}\rfloor}$, and:

$$
(2\lfloor\sqrt{n}\rfloor-1) q^{\lfloor\sqrt{n}\rfloor-1} \leq \sum_{k=1}^{\lfloor\sqrt{n}\rfloor-1}(2 k+1) q^{k} \leq \sum_{k=1}^{n} q^{\lfloor\sqrt{k}\rfloor} \leq \sum_{k=1}^{\lfloor\sqrt{n}\rfloor}(2 k+1) q^{k} \leq(2\lfloor\sqrt{n}\rfloor+1) q^{\lfloor\sqrt{n}\rfloor+1},
$$

the proposition follows.

\subsubsection{Acceptable segments}

Definition 13. Denote:

$$
\begin{aligned}
\Gamma_{[i, j]}^{t} & =\left\{x \in \mathcal{A}^{\mathbb{Z}} \mid[i, j] \text { is a segment of } F^{t}(x)\right\} \\
\Gamma_{l, k}^{t} & =\left\{x \in \mathcal{A}^{\mathbb{Z}}:[0, l] \text { is included in a segment of } F^{t}(x) \text { of length } k\right\} \\
& =\bigsqcup_{i=-k+\ell+1}^{0} \Gamma_{[i, i+k+1]}^{t} \quad \text { (disjoint union) } \\
\text { and } \quad \Gamma_{l, \geq k}^{t} & =\bigsqcup_{i \geq k} \Gamma_{l, i}^{t} .
\end{aligned}
$$

Proposition 8 (Lower bound).

$$
\text { Let } \mu \in \mathcal{M}_{\sigma-\text { erg }}^{\text {full }}\left(\mathcal{A}^{\mathbb{Z}}\right) \text {. For all } l \in \mathbb{N} \text {, one has } \mu\left(\Gamma_{l, \geq n}^{T_{n}}\right) \underset{n \rightarrow \infty}{\longrightarrow} 1 \text {. }
$$

Proof. $T_{n}=T_{n}^{\prime}$ for $n$ large enough, so we do the proof for $T_{n}^{\prime}$. Since $\mu$ has full support,

$$
\mu\left(x \in \mathcal{A}^{\mathbb{Z}}: x_{0}=\mathrm{I} \text { and } x_{i} \neq \mathrm{I} \text { for all } i \in\{-2,-1,1,2, \ldots, n\}\right) \neq 0 .
$$

By $\sigma$-ergodicity of $\mu$, segments of length larger than $n$ appear at time 0 in $\mu$-almost all configurations, and those segments survive up to time $T_{n}^{\prime}$ by construction. In particular, $F_{*}^{T_{n}^{\prime}} \mu([[\mathrm{I}]) \neq 0$.

By $\sigma$-ergodicity of $F_{*}^{T_{n}^{\prime}} \mu$, the cell 0 is $\mu$-almost surely included in some segment at time $T_{n}^{\prime}$, and this segment has length larger than $n$ by definition of $T_{n}^{\prime}$. By $\sigma$-invariance, the probability that $[0, l]$ crosses a border of the segment tends to 0 as $n$ tends to infinity.

Definition 14. Let $x \in \mathcal{A}^{\mathbb{Z}},[i, j]$ a segment at time $t \in\left[T_{n}, T_{n+1}\right]$. It is acceptable if $j-i-1 \leq$ $K_{n}=\sqrt{T_{n+1}-T_{n}}$. For $n$ large enough, $K_{n}=q^{\frac{\lfloor\sqrt{n}\rfloor}{2}}$.

Proposition 9 (Upper bound). Let $\mu \in \mathcal{M}_{\sigma-m i x}^{f u l l}\left(\mathcal{A}^{\mathbb{Z}}\right)$. One has $\mu\left(\Gamma_{l, \geq K_{n}}^{T_{n}}\right) \underset{n \rightarrow \infty}{\longrightarrow} 0$, that is to say:

$$
\mu\left(\left\{x \in \mathcal{A}^{\mathbb{Z}}:[0, l] \text { is in an acceptable segment of } F^{t}(x)\right\}\right) \underset{t \rightarrow \infty}{\longrightarrow} 1
$$

and the rate of convergence is exponential.

Proof. Again, $T_{n}=T_{n}^{\prime}$ for $n$ large enough, so we do the proof for $T_{n}^{\prime}$. Any segment at time $T_{n}^{\prime}$ corresponds to a segment larger than $n$ merged with 0 or more consecutive segments of length $n$ at time $T_{n-1}^{\prime}$ (only the left wall of segments of size $n$ are destroyed at time $T_{n}^{\prime}$ ). See Figure 9 for an illustration of this decomposition. Therefore we define:

$\Delta_{n, \alpha}^{t}=\left\{x \in \mathcal{A}^{\mathbb{Z}}\right.$ : starting from 0 there is a strip of $\alpha$ consecutive segments of size $n$ in $\left.F^{t}(x)\right\}$. 
First we bound the value of $\mu\left(\Delta_{n, \alpha}^{t}\right)$. For any $m>0$, by considering one symbol out of every $m$ :

$$
\begin{aligned}
\mu\left(\Delta_{n, \alpha}^{t}\right) & \leq \mu\left(\bigcap_{i=0}^{\alpha} \sigma^{i n}([[\mathrm{I}]])\right) \\
& \leq \mu\left(\bigcap_{i=0}^{\left\lfloor\frac{\alpha}{m}\right\rfloor} \sigma^{i n \cdot m}([[\mathrm{I}]))\right. \\
& \leq\left(1+\psi_{\mu}(m n)\right)^{\left\lfloor\frac{\alpha}{m}\right\rfloor} \mu\left([[\mathrm{I}])^{\left\lfloor\frac{\alpha}{m}\right\rfloor+1},\right.
\end{aligned}
$$

where $\psi_{\mu}$ are the weak mixing coefficients of $\mu$ as defined in Section 1.2.1.

Now take $x$ such that $[0, l]$ is included in a segment longer than $k$ at time $T_{n}^{\prime}$. As we said before, this segment is issued from the merging of one segment with 0 or more segments of length $n-1$ at time $T_{n-1}^{\prime}$. Take any $L>2 n$ and distinguish the two following cases concerning the segments at time $T_{n-1}^{\prime}$ it is issued from:

- There were less than $\left\lfloor\frac{L}{n}\right\rfloor$ segments of length $n$ : then the other segment is larger than $k-L$. By shifting the configuration by $L-l$ cells at most, we can ensure that $[0, l]$ is included in this segment at time $T_{n-1}^{\prime}$.

- There were more than $\left\lfloor\frac{L}{n}\right\rfloor$ segments of length $n$. Therefore there is a strip of $\left\lfloor\frac{L}{n}\right\rfloor$ segments of length $n$ starting at some $j \in[-k, k]$.

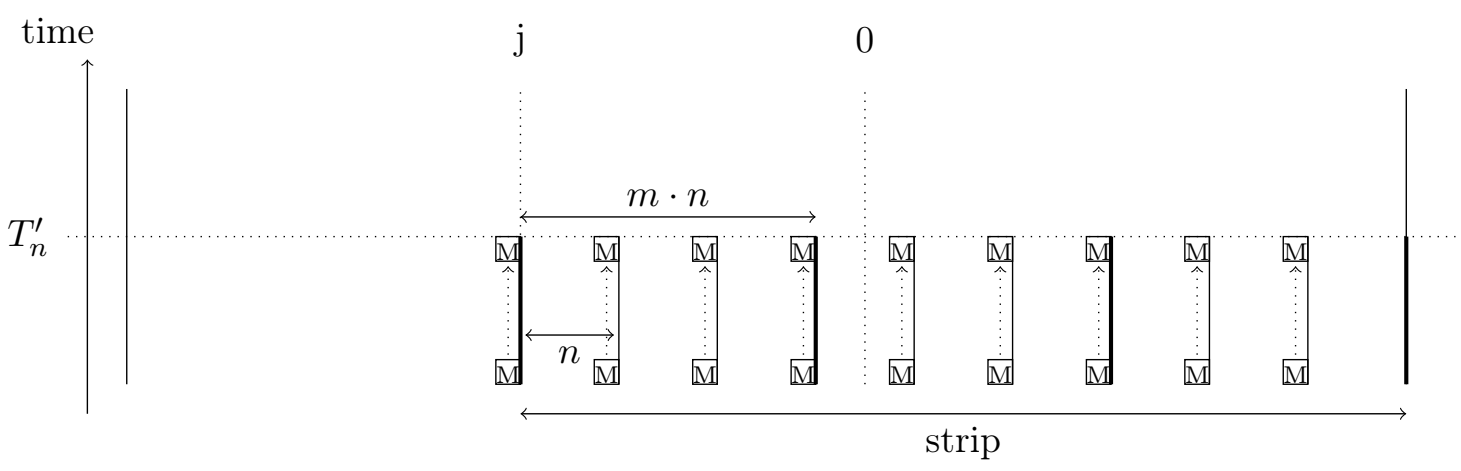

FiguRE 9. Illustration of the proof of Proposition 9 with $\alpha=9$ and $m=3$.

In other words,

$$
\Gamma_{l, \geq k}^{T_{n}^{\prime}} \subset \bigcup_{i=-L+l}^{0} \sigma^{i}\left(\Gamma_{l, \geq k-L}^{T_{n-1}^{\prime}}\right) \cup \bigcup_{j=-k+1}^{k-1} \sigma^{j}\left(\Delta_{n,\left\lfloor\frac{L}{n}\right\rfloor}^{T_{n-1}^{\prime}}\right) .
$$

From which it follows:

$$
\mu\left(\Gamma_{l, \geq k}^{T_{n}^{\prime}}\right) \leq L \mu\left(\Gamma_{l, \geq k-L}^{T_{n-1}^{\prime}}\right)+2 k \mu\left(\Delta_{n,\left\lfloor\frac{L}{n}\right\rfloor}^{T_{n-1}^{\prime}}\right)
$$


Now take an arbitrary $n_{0}>0$ and a constant $M \geq n_{0}$. For any $n \leq n_{0}$ and $k \geq L$, Using (11) with $m=\left\lceil\frac{M}{n}\right\rceil$ inside equation (2) yields:

$$
\begin{aligned}
\mu\left(\Gamma_{l, \geq k}^{T_{n}^{\prime}}\right) & \leq L \mu\left(\Gamma_{l, \geq k-L}^{T_{n-1}^{\prime}}\right)+2 k\left[1+\psi_{\mu}\left(n \cdot\left\lceil\frac{M}{n}\right\rceil\right)\right]^{\frac{L}{M}} \mu\left([[\mathrm{I}])^{\frac{L}{M}+1}\right. \\
& \leq L \mu\left(\Gamma_{l, \geq k-L}^{T_{n-1}^{\prime}}\right)+2 k\left[\left(1+\psi_{\mu}(M)\right) \mu([[\mathrm{I}])]^{\frac{L}{M}}\right.
\end{aligned}
$$

Applying this equation inductively, and assuming $k \geq n_{0} L$, we obtain:

$$
\mu\left(\Gamma_{l, \geq k}^{T_{n_{0}}^{\prime}}\right) \leq L^{n_{0}} \mu\left(\Gamma_{l, \geq k-n_{0} L}^{0}\right)+2 k n_{0}\left[\left(1+\psi_{\mu}(M)\right) \mu([[\mathrm{I}])]^{\frac{L}{M}}\right.
$$

For the first component of the right-hand term, we have:

$$
\begin{aligned}
\mu\left(\Gamma_{l, \geq k-n_{0} L}^{0}(x)\right) & \left.\leq \mu\left(\mathcal{A}^{\mathbb{Z}} \backslash \bigcap_{j=-k+n_{0} L}^{-1} \bigcup_{i=0}^{k-n_{0} L}[\mathrm{I}]\right]_{j+i}\right) \\
& \leq \mu\left(\bigcup_{j=-k+n_{0} L}^{-1} \bigcap_{i=0}^{\left\lfloor\frac{k-n_{0} L}{n_{0}}\right\rfloor}[\mathcal{A} \backslash[\mathrm{I}]]_{j+i n_{0}}\right) \\
& \leq\left(k-n_{0} L\right)\left(1+\psi_{\mu}\left(n_{0}\right)\right)^{\left\lfloor\frac{k-n_{0} L}{n_{0}}\right\rfloor} \mu([\mathcal{A} \backslash \mathrm{I}])^{\left\lfloor\frac{k-n_{0} L}{n_{0}}\right\rfloor+1}
\end{aligned}
$$

the second line being obtained by considering one symbol out of every $n_{0}$. To conclude, we fix the values $M=n_{0}, L=n_{0}^{2} \sqrt{n}_{0}$, and $k=K_{n_{0}}=\sqrt{T_{n_{0}+1}-T_{n_{0}}}$. Since $\psi_{\mu}(n) \rightarrow 0$ and Equation (3) holds for any $n_{0}$, we have $\mu\left(\Gamma_{l, \geq K_{n}}^{T_{n}^{\prime}}\right) \underset{n \rightarrow \infty}{\longrightarrow} 0$ and the rate of convergence is exponential.

\subsubsection{Density of auxiliary states}

By auxiliary state, we mean any element of $\mathcal{A} \backslash \mathcal{B}$, that is to say $\mathrm{I}, \mathrm{W}$ and any element of $\mathcal{A}$ which is not of the form $(b, \#, \#, \#, \#, \#)$.

Proposition 10. Fort large enough, an acceptable segment is formatted and contains only initialised processes.

Proof. In a segment of length $k$, Fact 5 ensures that the segment is formatted if $t \geq k(1+\log k)$. All remaining uninitialised processes may take up to $k$ more steps to be erased.

When $T_{n} \leq t<T_{n+1}$, for an acceptable segment of length $k$, we have $k(2+\log k) \leq K_{n}(2+$ $\left.\log \left(K_{n}\right)\right)=o\left(T_{n}\right)$ by Proposition 7 . Taking $n$ large enough, we conclude.

Proposition 11. Let $\mu \in \mathcal{M}_{\sigma-\text { erg }}^{\text {full }}\left(\mathcal{A}^{\mathbb{Z}}\right)$ and $u \in \mathcal{B}^{[0, \ell]}$ for some fixed $\ell$. For a given length $k$ such that $n+1 \leq k \leq K_{n}$, we have:

- If $t \in\left[T_{n}+k, T_{n+1}\right]$,

$$
\left|\mu\left(F^{-t}\left([u]_{0}\right) \mid \Gamma_{\ell, k}^{T_{n}}\right)-\widehat{\delta_{w_{n}}}([u])\right|=O\left(\frac{1}{\sqrt{n}}\right) ;
$$

- If $t \in\left[T_{n}, T_{n}+k\right]$,

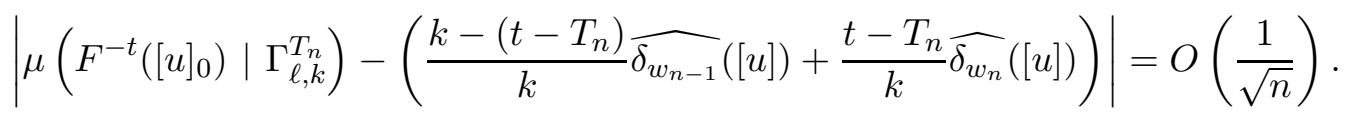

Proof. Take $x \in \Gamma_{[-1, k]}^{T_{n}}$. Since a segment of length $k$ with $n+1 \leq k \leq K_{n}$ is acceptable, it is formatted, and any uninitialised symbol has been destroyed. Since $\left|w_{n}\right|=O(\sqrt{n})$ (the length of the output is smaller than the computing space), the copying process uses $O(\sqrt{n})$ auxiliary cells. 


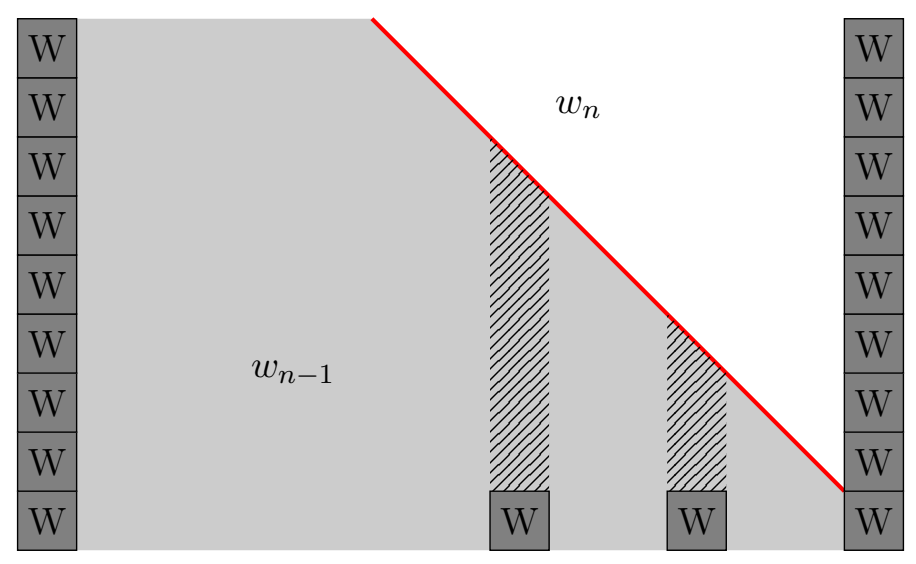

FIgURE 10. Illustration of Proposition 11, The output is not correctly written in dashed areas because of the destruction of a wall.

First point: The tail of the copying process progresses at speed one, so at time $T_{n}+k$ the copy of $w_{n}$ is finished, and until time $T_{n+1}$ the segment only contains copies of $w_{n}$ except for the time counter, computation and merging counter area $(O(\sqrt{n})$ cells) and a merging signal (one cell).

Therefore for all $x \in \Gamma_{[-1, k]}^{T_{n}}$, one has $\left|\operatorname{Freq}\left(u, F^{t}(x)_{[0, k-1]}\right)-\widehat{\delta_{w_{n}}}([u])\right|=\frac{O(\sqrt{n})}{k}=O\left(\frac{1}{\sqrt{n}}\right)$, taking into account the last copy of $w_{n}$ in the segment which can be incomplete $\left(\left|w_{n}\right| \leq \sqrt{n}\right)$, and since $k \geq n$. Thus we have:

$$
\left|\frac{1}{k} \sum_{i=0}^{k-1} \mu\left(F^{-t}\left([u]_{i}\right) \mid \Gamma_{[-1, k]}^{T_{n}}\right)-\widehat{\delta_{w_{n}}}([u])\right|=O\left(\frac{1}{\sqrt{n}}\right) .
$$

To conclude,

$$
\begin{aligned}
\mu\left(F^{-t}\left([u]_{0}\right) \mid \Gamma_{\ell, k}^{T_{n}}\right) & =\sum_{i=-k+\ell}^{-1} \mu\left(F^{-t}\left([u]_{0}\right) \mid \Gamma_{[-1-i, k-i]}^{T_{n}}\right) \cdot \mu\left(\Gamma_{[-1-i, k-i]}^{T_{n}} \mid \Gamma_{\ell, k}^{T_{n}}\right) \\
& =\frac{1}{k-\ell} \sum_{i=1}^{k-\ell} \mu\left(F^{-t}\left([u]_{0}\right) \mid \Gamma_{[i-1, i+k]}^{T_{n}}\right) \\
& =\frac{1}{k-\ell} \sum_{i=1}^{k-\ell} \mu\left(F^{-t}\left([u]_{i}\right) \mid \Gamma_{[-1, k]}^{T_{n}}\right)
\end{aligned}
$$

where the last two lines are by $\sigma$-invariance of $\mu$. Since each term is at distance $O\left(\frac{1}{\sqrt{n}}\right)$ of $\widehat{\delta_{w_{n}}}([u])$, the result follows.

Second point: When $t \in\left[T_{n}, T_{n}+k\right]$, the copy is still taking place, with $t-T_{n}$ cells containing copies of $w_{n}$ and the rest containing copies of $w_{n-1}$, except for except for $O(\sqrt{n})$ various auxiliary states, and possibly defects when a wall has been destroyed at time $T_{n}$ (there are at most $\frac{k}{n}$ of them). Therefore

$$
\mid \text { Freq }\left(u, F^{t}(x)_{[0, k-1]}\right)-\left(\frac{k-\left(t-T_{n}\right) \widehat{\delta_{w_{n-1}}}}{k}([u])+\frac{t-T_{n}}{k} \widehat{\delta_{w_{n}}}([u])\right) \mid=\frac{1}{k} O(\sqrt{n}) \cdot \frac{k}{n}=O\left(\frac{1}{\sqrt{n}}\right),
$$

since $k \geq n$. Using the same reasoning as the first point, we conclude. 


\subsubsection{Proof of Theorem 1 - first point}

We prove the following: for a given computable sequence of words $\left(w_{n}\right)_{n \in \mathbb{N}}$, the CA $F$ that we described above satisfies that for any measure $\mu \in \mathcal{M}_{\sigma-\operatorname{mix}}^{\text {full }}\left(\mathcal{A}^{\mathbb{Z}}\right), \mathcal{V}(F, \mu)=\mathcal{V}\left(\left(w_{n}\right)_{n \in \mathbb{N}}\right)$.

Let $\mu \in \mathcal{M}_{\sigma-\text { mix }}^{\text {full }}\left(\mathcal{A}^{\mathbb{Z}}\right)$ and $u \in \mathcal{B}^{[0, \ell]}$. By Propositions 8 and 9, $\mu\left(\bigcup_{k=n+1}^{K_{n}} \Gamma_{\ell, k}^{T_{n}^{\prime}}\right) \underset{n \rightarrow \infty}{\longrightarrow} 1$ exponentially fast, and $\Gamma_{\ell, k}^{t}=\Gamma_{\ell, k}^{T_{n}^{\prime}}$ for $t \in\left[T_{n}^{\prime}, T_{n+1}^{\prime}-1\right]$. Therefore:

$$
\exists C>0, \max _{T_{n} \leq t<T_{n+1}}\left|F_{*}^{t} \mu([u])-\sum_{k=n+1}^{K_{n}} \mu\left(F^{-t}([u]) \mid \Gamma_{\ell, k}^{t}\right) \mu\left(\Gamma_{\ell, k}^{t}\right)\right|=O\left(e^{-C n}\right) .
$$

Take $n$ large enough that $T_{n}=T_{n}^{\prime}$. By Proposition 11,

$$
\begin{aligned}
\max _{T_{n}^{\prime} \leq t<T_{n+1}^{\prime}} \mid F_{*}^{t} \mu([u])-\sum_{k=n+1}^{K_{n}} \mu\left(\Gamma_{\ell, k}^{T_{n}}\right) & \left(\max \left(0, \frac{k-\left(t-T_{n}\right)}{k}\right) \widehat{\delta_{w_{n-1}}}([u])\right. \\
& \left.+\min \left(1, \frac{t-T_{n}}{k}\right) \widehat{\delta_{w_{n}}}([u])\right) \mid=O\left(\frac{1}{\sqrt{n}}\right) .
\end{aligned}
$$

Let $f_{n}$ be the piecewise affine function defined by:

$$
\begin{aligned}
f_{n}:\left[T_{n}, T_{n+1}\right] & \longrightarrow[0,1] \\
t & \longmapsto \sum_{k=n+1}^{K_{n}} \min \left(1, \frac{t-T_{n}}{k}\right) \mu\left(\Gamma_{\ell, k}^{T_{n}}\right)+\frac{t-T_{n}}{T_{n+1}-T_{n}} \mu\left(\Gamma_{\ell,>K_{n}}^{T_{n}}\right) .
\end{aligned}
$$

The second term is chosen so that $f_{n}\left(T_{n}\right)=0$ and $f_{n}\left(T_{n+1}\right)=1$, but it converges to 0 exponentially fast and thus does not affect the equation by more than $O\left(\frac{1}{\sqrt{n}}\right)$. Therefore:

$$
\begin{gathered}
\max _{T_{n} \leq t<T_{n+1}}\left|F_{*}^{t} \mu([u])-\left(f_{n}(t) \widehat{\delta_{w_{n}}}([u])+\left(1-f_{n}(t)\right) \widehat{\delta_{w_{n-1}}}([u])\right)\right|=O\left(\frac{1}{\sqrt{n}}\right) . \\
\max _{T_{n} \leq t<T_{n+1}} d_{\mathcal{M}}\left(F_{*}^{t} \mu,\left[\widehat{\delta_{w_{n-1}}}, \widehat{\delta_{w_{n}}}\right]\right)=O\left(\frac{1}{\sqrt{n}}\right),
\end{gathered}
$$

so $\mathcal{V}(F, \mu) \subset \mathcal{V}\left(\left(w_{n}\right)_{n \in \mathbb{N}}\right)$. Since $f_{n}$ is $\frac{1}{n}$-Lipschitz on $\left[T_{n}, T_{n+1}\right]$, any $\nu \in\left[\widehat{\delta_{w_{n-1}}}, \widehat{\delta_{w_{n}}}\right]$ is at distance at most $\frac{1}{n}$ of an element of the form $\left(f_{n}(t) \widehat{\delta_{w_{n}}}+\left(1-f_{n}(t)\right) \widehat{\delta_{w_{n-1}}}\right)$ for $T_{n} \leq t<T_{n+1}$.

We conclude that $\mathcal{V}(F, \mu)=\mathcal{V}\left(\left(w_{n}\right)_{n \in \mathbb{N}}\right)$.

Rate of convergence. For clarity, assume that $w_{n}$ is computable in space $O(\sqrt{n})$ by repeating elements if necessary.

By Proposition $\left[7\right.$ we have $T_{n}=\Theta\left(\lfloor\sqrt{n}\rfloor q^{\lfloor\sqrt{n}\rfloor}\right)$ so, writing $n(t)$ the current value of $n$ at time $t$, we have $n(t)=\Theta\left(\log (t)^{2}\right)$ and $O\left(\frac{1}{\sqrt{n(t)}}\right)=O\left(\frac{1}{\log t}\right)$.

We find that the rate of convergence is:

$$
\begin{aligned}
d_{\mathcal{M}}\left(F_{*}^{t} \mu, \mathcal{V}\left(\left(w_{n}\right)_{n \in \mathbb{N}}\right)\right) & \leq d_{\mathcal{M}}\left(F_{*}^{t} \mu,\left[\widehat{\delta_{w_{n(t)-1}}}, \widehat{\delta_{w_{n}(t)}}\right]\right)+\underset{\nu \in\left[\widehat{\left.\delta_{w_{n(t)-1}}, \widehat{\delta_{w_{n}(t)}}\right]}\right.}{\sup } d_{\mathcal{M}}\left(\nu, \mathcal{V}\left(\left(w_{n}\right)_{n \in \mathbb{N}}\right)\right) \\
& =O\left(\frac{1}{\log (t)}\right)+\sup \left\{d_{\mathcal{M}}\left(\nu, \mathcal{V}\left(\left(w_{n}\right)_{n \in \mathbb{N}}\right)\right): \nu \in \bigcup_{n \geq n(t)}\left[\widehat{\delta_{w_{n}}}, \widehat{\delta_{w_{n+1}}}\right]\right\},
\end{aligned}
$$

by the last proof. 


\subsubsection{Proof of Theorem 1 - second point}

Now we treat the case where $\mathcal{V}\left(\left(w_{i}\right)_{i \in \mathbb{N}}\right)=\{\nu\}$. Let $F$ be the cellular automaton associated with this sequence as described above, and consider $\mu \in \mathcal{M}_{\sigma-\operatorname{erg}}^{\text {full }}\left(\mathcal{A}^{\mathbb{Z}}\right)$. Since $\mu$ is not assumed to be $\psi$-mixing, Proposition 9 does not apply, and there is no guarantee most segments are acceptable. However large segments are still rare; more precisely, $\mu\left(\Gamma_{0, \geq k}^{t}\right) \underset{k \rightarrow \infty}{\longrightarrow} 0$ for all $t$ since all sets $\Gamma_{0, k}^{t}$ are disjoint.

Claim 1: $\quad F_{*}^{t} \mu([\mathcal{A} \backslash \mathcal{B}]) \underset{t \rightarrow \infty}{\longrightarrow} 0$, i.e., the density of auxiliary states tends to 0 .

Proof. Suppose we are in an initial segment of length $k$. Detached time counters, Turing machines and merging counters initially present are destroyed in less than $k$ steps. Similarly, left merging signals and copy auxiliary states initially present progress at speed -1 , so they are destroyed before time $k$. Any uninitialised wall is destroyed after $k(1+\log k)$ steps at most, and any counter attached to it are destroyed after less than $k$ more steps. For all those states, the probability of apparition after time $k(2+\log k)$ is less than $\mu\left(\Gamma_{0, \geq k}^{0}\right) \underset{k \rightarrow \infty}{\longrightarrow} 0$.

At time $T_{n}^{\prime}$, all segments are longer than $n$, so the density of initialised walls and initialised auxiliary states inside each segment is $O\left(\frac{\sqrt{n}}{n}\right)$.

Only uninitialised formatting counters and right merging signals remain. Inside each segment, call non-formatted area the interval between the initialised formatting counter of the left wall and the rightmost cell containing one of those two states. At each step, this area decreases by one cell to its left but may grow by one cell to its right as a counter or signal progresses. Notice that merging with other segments cannot increase this area since segments of length $n$ at time $T_{n}$ are formatted (see Figure 11).

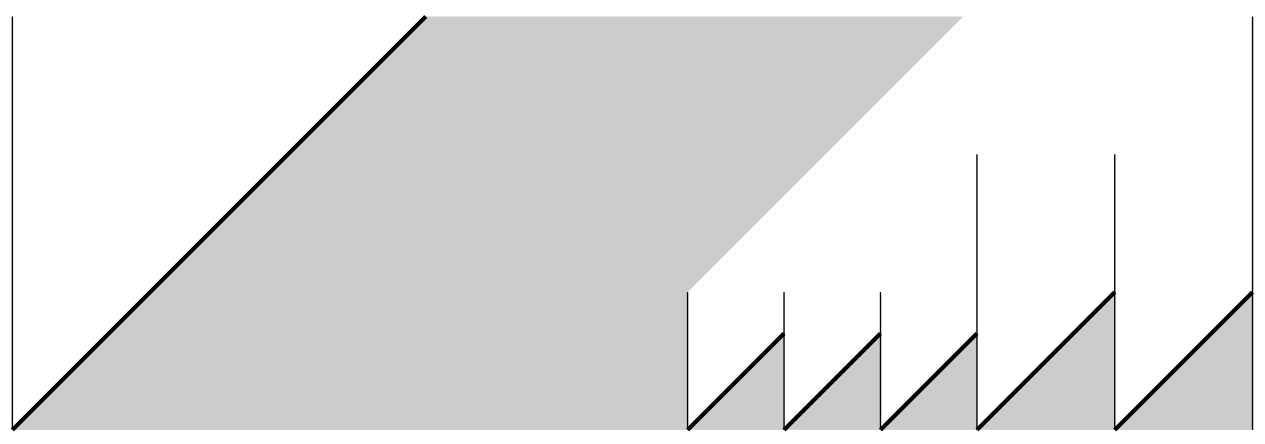

FiguRE 11. Illustration of the last part of the proof of Claim 1. Slanted lines are formatting counters and grey areas are potentially non-formatted.

Therefore, a segment at time $T_{n}$ can contain a non-formatted area longer than $\sqrt{n}$ only if it is issued from a segment longer than $\sqrt{n}$ initially. Other segments have a non-formatted area smaller than $\sqrt{n}$ for a length larger than $n$. By $\sigma$-invariance,

$$
\mu\left(\left\{x \in \mathcal{A}^{\mathbb{Z}} \mid x_{0} \text { is in a non-formatted area }\right\}\right) \leq \frac{\sqrt{n}}{n}+\mu\left(\Gamma_{0, \geq \sqrt{n}}^{0}\right) \underset{n \rightarrow \infty}{\longrightarrow} 0 .
$$

Therefore, for $a \in \mathcal{A} \backslash \mathcal{B}$, we have $F_{*}^{t} \mu([a]) \underset{t \rightarrow \infty}{\rightarrow} 0$.

$\diamond$ Claim 1

Claim 2: For any $n \in \mathbb{N}, d_{\mathcal{M}}\left(F_{*}^{t} \mu, \operatorname{Conv}\left(\left(\widehat{\delta_{w_{i}}}\right)_{i \geq n}\right)\right) \underset{t \rightarrow \infty}{\longrightarrow} 0$, where $\operatorname{Conv}(X)$ is the convex hull of the set $X$. 
Proof. Consider a segment of length $k$ at time $T_{n}$. At time $T_{n}+k$ the copying process for $w_{n}$ is finished, but since the segment is not necessarily acceptable, other copying processes may have started in the meanwhile (see Figure 12). Therefore, the segment contains:

- auxiliary states, with negligible frequency;

- strips containing repeated copies of $w_{n}$, then $w_{n+1}, w_{n+2} \ldots$ separated by ongoing copy processes and the frequency of auxiliary copy states being negligible.

Consider a segment of size $k$ at time $T_{n}$ in the positions $[i, i+k]$. At time $T_{n}+k$ it is filled with copies of $w_{n}$. When $t \geq T_{n}+k$, the positions $[i, i+k]$ contain a succession of stripes containing $w_{N}, w_{N+1}, \ldots$ with $N \geq n$ plus a negligible part of auxiliary states and defects. The strip containing $w_{i}$ is larger than $i$ since this word is produced in a segment of size larger than $i$. One deduces that $\mu\left(F^{-\left(T_{n}+t\right)}\left([u]_{0}\right) \mid \Gamma_{\ell, k}^{T_{n}}\right)$ is quite near of $\operatorname{Conv}\left(\left(\widehat{\delta_{w_{i}}}([u])\right)_{i \geq n}\right)$ for $t \geq k$. Since $\mu\left(\Gamma_{0, \geq k}^{T_{n}}\right) \underset{k \rightarrow \infty}{\longrightarrow} 0$, we have

$$
d_{\mathcal{M}}\left(F_{*}^{T_{n}+t} \mu, \operatorname{Conv}\left(\left(\widehat{\delta_{w_{i}}}\right)_{i \geq n}\right)\right) \underset{t \rightarrow \infty}{\longrightarrow} 0
$$

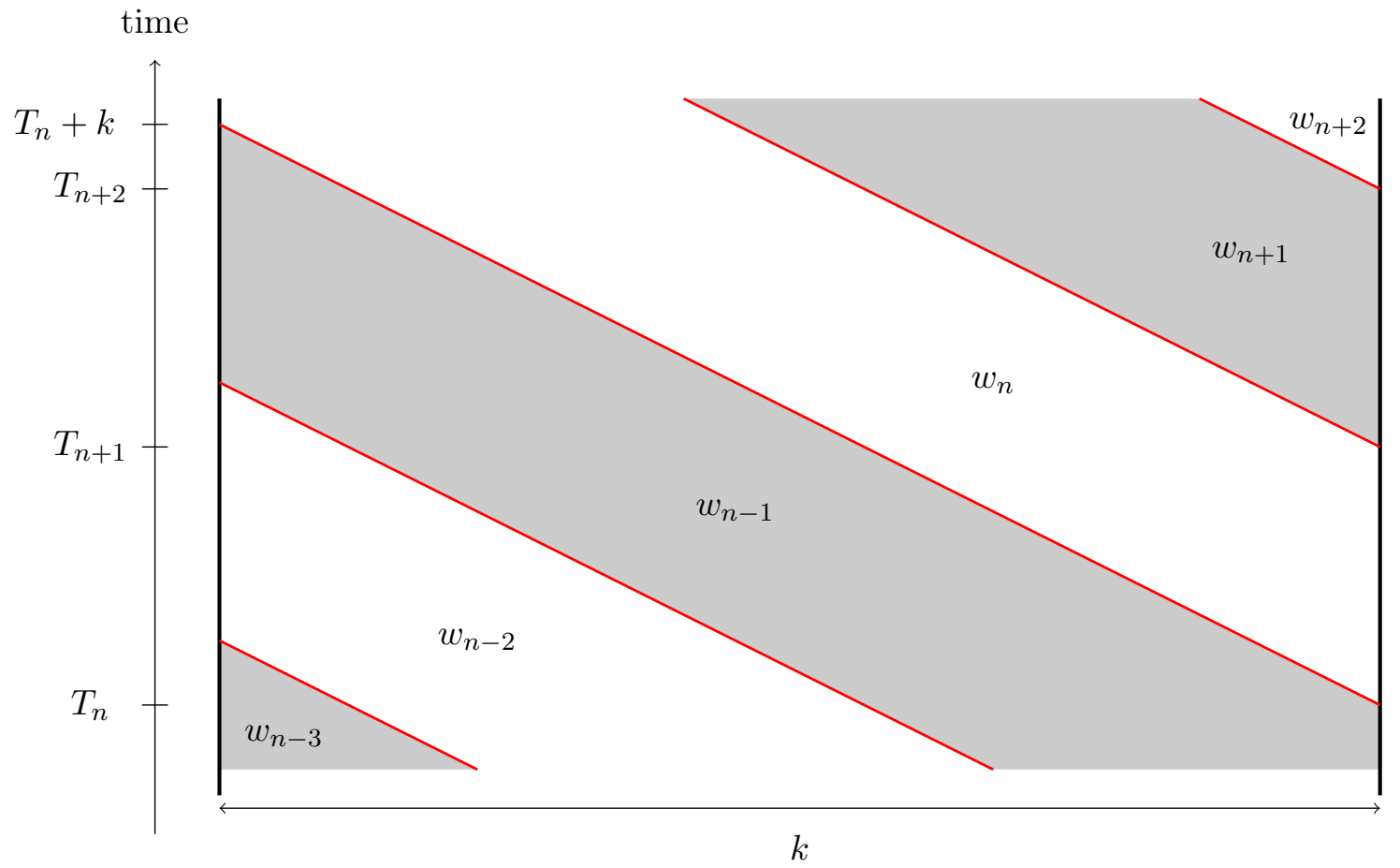

Figure 12. Illustration of Claim 2. When $t>T_{n}+k$, a segment of length $k$ is a succession of stripes containing $w_{n}, w_{n+1}, \ldots$ plus a negligible part of auxiliary states and defects.

The second point of the Theorem 1 follows easily from Claim 2.

Remark. It does not follow from the last claim that the sequence $\left(F_{*}^{t} \mu\right)$ is close to any of the $\widehat{\delta_{w_{i}}}$ at any point, which is the reason why the result holds only for a single measure. Controlling the length of the segments as needed in the proof of the first point requires $\psi$-mixing. 


\section{REMOVing THE AUXILIARY STATES}

Before stating consequences of Theorem 1, we consider in this section the case where the cellular automaton does not use auxiliary states, that is, $\mathcal{A}=\mathcal{B}$. A straightforward extension is impossible: if $\nu$ is a full support measure, and $F: \mathcal{A}^{\mathbb{Z}} \rightarrow \mathcal{A}^{\mathbb{Z}}$ a cellular automaton such that $F_{*}^{t} \mu \rightarrow \nu$ for some initial measure $\mu$, then $F$ is a surjective automaton which leaves the uniform Bernoulli measure invariant. Therefore, starting from the uniform measure, $F$ can only reach the uniform Bernoulli measure.

However, if the limit measure does not have full support, the previous results can be extended by using a word not charged by the measure to encode the auxiliary states in some sense.

Theorem 2. Let $\left(w_{n}\right)_{n \in \mathbb{N}}$ be a uniformly computable sequence of words of $\mathcal{B}^{*}$, where $\mathcal{B}$ is a finite alphabet, and assume there exists a word $u$ that does not appear as subwords in any of the $w_{n}$. Then there is a cellular automaton $F: \mathcal{B}^{\mathbb{Z}} \rightarrow \mathcal{B}^{\mathbb{Z}}$ such that for any measure $\mu \in \mathcal{M}_{\sigma-\text { mix }}^{\text {full }}\left(\mathcal{B}^{\mathbb{Z}}\right)$, $\mathcal{V}(F, \mu)=\mathcal{V}\left(\left(w_{n}\right)_{n \in \mathbb{N}}\right)$.

However, because of the destructive nature of the formatting counter in the construction, the proof in Section 3.5 .4 cannot be adapted and we cannot weaken the hypothesis to $\mu \in \mathcal{M}_{\sigma-\operatorname{erg}}^{\text {full }}\left(\mathcal{B}^{\mathbb{Z}}\right)$ when $\mathcal{K}$ is a singleton.

Proof. Let $\mathcal{A} \supset \mathcal{B}$ be the alphabet and $F$ the $\mathrm{CA}$ associated to the sequence $\left(w_{n}\right)_{n \in \mathbb{N}}$ by Theorem 1 , Our aim is to provide an encoding of any configuration of $\mathcal{A}^{\mathbb{Z}}$ in $\mathcal{B}^{\mathbb{Z}}$ and a cellular automaton $F^{\prime}$ that behaves similarly to $F$ after encoding.

Denote $U_{d} \subset \mathcal{B}^{d}$ the set of words of length $d$ with prefix $u$, that do not contain $u$ as subword (except at the first letter), and that do not end with a prefix of $u$. $\# U_{d} \underset{d \rightarrow \infty}{\longrightarrow} \infty$, so for $d$ large enough, we can find an injection $\varphi: \mathcal{A} \backslash \mathcal{B} \rightarrow U_{d}$ (encoding the auxiliary states), and we extend it by putting $\varphi=I d$ on $\mathcal{B}$. For a finite word, we define $\varphi\left(u_{1} \ldots u_{n}\right)=\varphi\left(u_{1}\right) \ldots \varphi\left(u_{n}\right)$, and this can be naturally extended further to configurations $\Phi: \mathcal{A}^{\mathbb{Z}} \longmapsto \mathcal{B}^{\mathbb{Z}}$ by considering that $\varphi\left(a_{0}\right)$ starts on the column zero. Notice that this encoding is not $\sigma$-invariant.

Let $\mathbf{T} \subset \mathcal{A}^{\mathbb{Z}}$ be the set of configurations such that the word $u$ does not appear on the main layer ( $\mathbf{T}$ is a subshift of finite type). Since $u$ marks unambiguously the beginning of a word of $\varphi(\mathcal{A} \backslash \mathcal{B})$, the restriction $\Phi: \mathbf{T} \rightarrow \mathcal{B}^{\mathbb{Z}}$ is injective.

We can define locally a decoding $\Psi: \Phi(\mathbf{T}) \rightarrow \mathbf{T}$ such that $\Psi \circ \Phi=\mathrm{Id}$, by looking $d$ cells to the right for occurrences of $u$. If $u$ appears, we are an output cell, that is, the image by $\varphi$ of a single letter $b \in \mathcal{B}$ (corresponding to (b,\#,\#,\#,\#,\#) for $b \in \mathcal{B}$ in the previous construction); otherwise, we belong in an auxiliary cluster, the image by $\varphi$ of a letter $A \backslash B$ that occupy $d$ cells while containing one letter of output. See Figure 13 for an example.

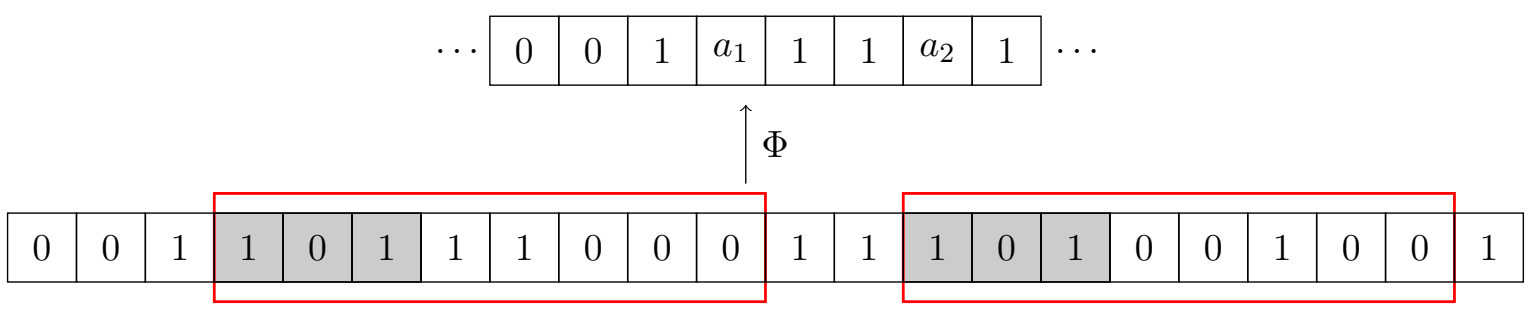

FigURE 13. Encoding of the auxiliary states with $u=101$ and $d=3$. In this case $U_{d} \subset 101 \cdot \mathcal{A}^{3} \cdot 00$. 
Intuitively, we want to build a cellular automaton that behaves similarly as the automaton defined in Theorem 1, where elements $(b, \#, \#, \#, \#, \#)$ are represented by output cells and all other elements by auxiliary clusters. However, $\Phi$ and $\Psi$ are not $\sigma$-invariant, so $\Phi \circ F \circ \Psi$ is not a cellular automaton. Instead, we build manually a cellular automaton on $\mathcal{B}^{\mathbb{Z}}$ that behaves in roughly the same way as $\Phi \circ F \circ \Psi$.

Provided the neighbourhood is larger than $[-4 d, 4 d]$, each cell can "read" the cluster in which it belongs, and the three clusters to its right and left. At time 0, if a word $u$ is not the prefix of a word of $U_{d}$, it is replaced by a word $b^{d}$ and can never be created again. To avoid creating an auxiliary cluster by mistake, we fix to this purpose a letter $b \in \mathcal{B}$ such that $b^{d} \notin U_{d}$. Similarly, auxiliary clusters that are destroyed for any reason leave behind them output $b$ cells.

Remark. For clarity, in all diagrams of this section, we suppose that $\mathcal{B}=\{0,1\}, d=3$ (it would be much larger in a real implementation) and we represent auxiliary clusters as blocks with layers, instead of words from $\mathcal{B}^{d}$. Also we fix $b=0$ in the definition above.

The different parts of the construction are modified in the following way.

- I and $\mathrm{W}$ clusters, time counters, and Turing machines have the same behaviour as in the previous construction. However, since the counters take more space, it is necessary to erase $3 d$ cells to the left and right of each $\mathrm{I}$ cluster at time 0.

\begin{tabular}{|c|c|c|c|c|c|c|c|c|c|c|}
\hline \multirow{2}{*}{ W } & \multirow{2}{*}{0} & \multirow{2}{*}{0} & \multicolumn{2}{|c|}{1} & \multirow{2}{*}{1} & \multirow{2}{*}{1} & \multirow{2}{*}{1} & \multirow{2}{*}{0} & \multirow{2}{*}{1} & \multirow{5}{*}{ copy } \\
\hline & & & & 0 & & & & & & \\
\hline \multirow{2}{*}{ W } & \multicolumn{3}{|c|}{1} & \multicolumn{2}{|c|}{1} & \multirow{2}{*}{1} & \multirow{2}{*}{1} & \multirow{2}{*}{0} & \multirow{2}{*}{1} & \\
\hline & & 0 & & & & & & & & \\
\hline \multirow[b]{2}{*}{$\mathrm{W}$} & \multirow{2}{*}{$?$} & \multirow{2}{*}{\multicolumn{2}{|c|}{0}} & & \multicolumn{2}{|l|}{1} & \multirow[b]{2}{*}{1} & \multirow{2}{*}{0} & \multirow{2}{*}{1} & \\
\hline & & & & & 1 & & & & & \\
\hline \multirow{2}{*}{ W } & \multirow{2}{*}{$?$} & \multirow{2}{*}{$?$} & \multirow{2}{*}{\multicolumn{2}{|c|}{$\frac{1}{1}$}} & \multicolumn{3}{|c|}{0} & \multirow{2}{*}{0} & \multirow{2}{*}{1} & \multirow{6}{*}{ main } \\
\hline & & & & & & 7 & & & & \\
\hline \multirow{2}{*}{$\mathrm{W}$} & \multirow{2}{*}{$?$} & \multirow{2}{*}{$?$} & \multirow{2}{*}{$?$} & \multicolumn{2}{|c|}{1} & \multicolumn{3}{|c|}{1} & 1 & \\
\hline & & & & & & & 0 & & 1 & \\
\hline W & $?$ & $?$ & $?$ & $?$ & 1 & & & 1 & & \\
\hline $\mathrm{v}$ & : & : & ! & ? & 0 & & & 1 & & \\
\hline
\end{tabular}

FIgURE 14. End of the copying process described in Figure 7, copying the word 1101.

- The tail of copying processes progresses to the left at speed one, and behaves normally as long as it does not meet another auxiliary state (see Figure 14). When the process has finished the copy, it is destroyed and leaves $b$ cells behind.

- Formatting counters progress to the right at speed $d$. This is too fast to keep information on the output layer, so the counter leaves behind output cells $b$ defined above. Any other signal it meets (e.g. copying process or length-measuring signal) is similarly erased.

- When close to a time counter, it may happen that the formatting counter cannot progress by $d$ cells exactly (see Figure 15). In this case, it is offset by less than $d$ cells, and formatting clusters separated by small offsets in this way are still considered to be the same counter for the rule of the automaton. The subsequent comparison process is unchanged.

- Merging signals which determine length of segments also progress at speed $d$. To avoid possible interactions with copying processes (similarly to the case of formatting counters), 


\begin{tabular}{|c|c|c|c|c|c|c|c|c|c|c|c|}
\hline 0 & 0 & 0 & 0 & 0 & 0 & 0 & 0 & 0 & $?$ & $\begin{array}{c}\text { time } \\
\text { formatting }\end{array}$ & $\begin{array}{c}\text { time } \\
\text { formatting }\end{array}$ \\
\hline 0 & 0 & 0 & 0 & 0 & 0 & formatting & $?$ & $\begin{array}{c}\text { time } \\
\text { formatting }\end{array}$ & time \\
\hline 0 & 0 & 0 & formatting & formatting & $?$ & time & time \\
\hline \begin{tabular}{|l|c|c|c|c|c|c|c|c|}
\hline formatting \\
\hline
\end{tabular} \\
\hline
\end{tabular}

FiguRE 15. A formatting counter gets offset when entering the time counter area. Notice the auxiliary clusters being replaced by output cells containing $b=0$.

\begin{tabular}{|c|c|c|c|c|c|c|c|c|c|c|}
\hline $\mathrm{W}$ & $?$ & \multicolumn{3}{|c|}{ 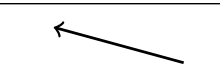 } & $?$ & $?$ & 0 & time & time & $\mathrm{W}$ \\
\hline W & $?$ & $?$ & $?$ & 0 & \multicolumn{3}{|c|}{ 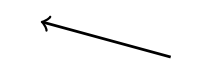 } & time & time & W \\
\hline W & $?$ & $?$ & $?$ & 0 & 0 & 0 & $?$ & time & time & $\mathrm{W}$ \\
\hline W & $?$ & $?$ & $?$ & 0 & 0 & 0 & $?$ & time & time & W \\
\hline W & $?$ & $?$ & $?$ & 0 & 0 & 0 & $?$ & time & 土infe & W \\
\hline $\mathrm{W}$ & $?$ & $?$ & $?$ & 0 & 0 & 0 & $?$ & 土ime & time & $\mathrm{W}$ \\
\hline W & $?$ & $?$ & $?$ & & & & $?$ & time & time & W \\
\hline $\mathrm{W}$ & & - & & $?$ & $?$ & $?$ & $?$ & time & time & $\mathrm{W}$ \\
\hline
\end{tabular}

Figure 16. Determination of length. Here $d=3, t_{0}=8$ and $o=1$, for a measured length of 13.

the determination of length starts only after the copy is finished. Thus a merging signal is only offset when entering the time counter area. After bouncing off the right wall, it returns to the left wall where its offset can be measured. If it takes $t_{0}$ steps to return with an offset of $\alpha$, then the segment has length $\frac{t_{0}}{2} \cdot d+\alpha$ (see Figure 16). This value is compared to $n$ and the rest of the process is not modified.

In this way, Propositions 9 and 10 still hold. We can check that at time $t$, with $T_{n} \leq t<T_{n+1}$, the copy process followed by the process of determination of length for segments of size $n+1$ still take less than $T_{n+1}-T_{n}$ steps. Furthermore, the frequency of auxiliary states is multiplied by a fixed constant $d$. Hence the proof in Section 3.5 .3 can be adapted, and the theorem follows. 


\section{Problems SOlved With This CONSTRUCTION}

In this section, we use Theorem 1 to solve various problems, starting with the characterisation of reachable limit measures and connected $\mu$-limit measure sets. We then consider the disconnected case, the case when auxiliary states are not allowed, Césàro mean convergence and consequences of these results for the decidability of asymptotic properties of cellular automata.

\subsection{Characterisation of reachable $\mu$-limit measures set}

\subsubsection{The connected case}

Reciprocals of the computable obstructions described in Section 2 follow directly from Theorem 1 ,

Corollary 1. Let $\nu \in \mathcal{M}_{\sigma}^{\Delta_{2} \text {-comp }}\left(\mathcal{B}^{\mathbb{Z}}\right)$ be a limit-computable measure. There is an alphabet $\mathcal{A} \supset \mathcal{B}$ and a cellular automaton $F: \mathcal{A}^{\mathbb{Z}} \rightarrow \mathcal{A}^{\mathbb{Z}}$ such that for any $\mu \in \mathcal{M}_{\sigma-\text { erg }}^{\text {full }}\left(\mathcal{A}^{\mathbb{Z}}\right)$, one has $F_{*}^{t} \mu \underset{t \rightarrow \infty}{\longrightarrow} \nu$.

Proof. Combine Proposition 1 with the second point of Theorem 1 .

Corollary 2. Let $\mathcal{K} \subset \mathcal{M}_{\sigma}\left(\mathcal{B}^{\mathbb{Z}}\right)$ be a compact, $\Pi_{2}$-computable and connected $\left(\Pi_{2}-C C C\right)$ subset of $\mathcal{M}_{\sigma}\left(\mathcal{B}^{\mathbb{Z}}\right)$. There is an alphabet $\mathcal{A} \supset \mathcal{B}$ and a cellular automaton $F: \mathcal{A}^{\mathbb{Z}} \rightarrow \mathcal{A}^{\mathbb{Z}}$ such that for any $\mu \in \mathcal{M}_{\sigma-\text { mix }}^{\text {full }}\left(\mathcal{A}^{\mathbb{Z}}\right)$, one has $\mathcal{V}(F, \mu)=\mathcal{K}$.

This is in particular a full characterisation of limit measures and connected $\mu$-limit measures sets that are reachable from some computable initial measure $\mu \in \mathcal{M}_{\sigma}\left(\mathcal{A}^{\mathbb{Z}}\right)$.

Proof. Combine Proposition 6 with the first point of Theorem 1.

For both of these statements, a rate of convergence is given in Theorem 1, and this rate depends partly of the quality of the approximation of the target measure or the target set by a uniformly computable sequence of computable measures supported by periodic orbits.

Open question 1. Can the rate of convergence be improved, or can we prove that this is the best possible rate?

The following corollary is the counterpart of Corollary 2 using Theorem 2 , Corollary 11 does not have a counterpart since its proof uses the second point of Theorem 1 .

Definition 15. A word $u \in \mathcal{A}^{*}$ is said to be not charged by a set $\mathcal{K} \in \mathcal{M}_{\sigma}\left(\mathcal{A}^{\mathbb{Z}}\right)$ if for all $\nu \in \mathcal{K}$, $\nu([u])=0$.

Corollary 3. Let $\mathcal{K} \subset \mathcal{M}_{\sigma}\left(\mathcal{B}^{\mathbb{Z}}\right)$ be a non-empty $\Pi_{2}-C C C$ subset of $\mathcal{M}_{\sigma}\left(\mathcal{B}^{\mathbb{Z}}\right)$ that does not charge a word $u \in \mathcal{B}^{*}$. Then there is a cellular automaton $F: \mathcal{B}^{\mathbb{Z}} \rightarrow \mathcal{B}^{\mathbb{Z}}$ such that for any measure $\mu \in \mathcal{M}_{\sigma-\text { mix }}^{\text {full }}\left(\mathcal{B}^{\mathbb{Z}}\right), \mathcal{V}(F, \mu)=\mathcal{K}$. In particular, any limit-computable measure which does not have full support can be obtained by this way.

Proof. Since $\mathcal{K}$ does not charge $u$, we can assume without loss of generality that no word in the uniformly computable sequence $\left(w_{n}\right)_{n \in \mathbb{N}}$ associated to $\mathcal{K}$ by Proposition 6 contain $u$ as subword. Indeed, if not we replace by an other word, this transformation does not have influence on $\mathcal{V}\left(\left(w_{n}\right)_{n \in \mathbb{N}}\right)$ since $u$ does not have. Thus Theorem 2 applies.

We leave open in particular the case of limit measures with full support which can happen only if $F$ is surjective. For Corollaries 3 and 6 , solving this case would imply to characterise the possible asymptotic behaviours of surjective automata. In this case a similar construction seems difficult since the state $\mathrm{W}$ which appear only in the initial configuration cannot be coded.

Open question 2. Which sets of measures can be reached at the limit by surjective cellular automata? 


\subsubsection{Towards the non-connected case}

In Corollary 2 the $\mu$-limit measures set is assumed to be connected. Indeed, in the construction of Theorem 1, each word $w_{n}$ is copied progressively on each segment, so that we reach the closure of an infinite polygonal path which is connected. However, non-connected $\mu$-limit measures sets also have some obstructions. For example, if $\mathcal{V}(F, \mu)$ is finite, we have the following proposition.

Proposition 12. Let $F: \mathcal{A}^{\mathbb{Z}} \rightarrow \mathcal{A}^{\mathbb{Z}}$ be a cellular automaton and $\mu \in \mathcal{M}_{\sigma}\left(\mathcal{A}^{\mathbb{Z}}\right)$ such that $\mathcal{V}(F, \mu)$ is finite. Then $F_{*}$ induces a cycle on $\mathcal{V}(F, \mu)$.

Proof. Let $d=\min \left\{d_{\mathcal{M}}\left(\nu, \nu^{\prime}\right): \nu, \nu^{\prime} \in \mathcal{V}(F, \mu)\right.$ with $\left.\nu \neq \nu^{\prime}\right\}>0$ and consider $\nu \in \mathcal{V}(F, \mu)$. It is possible to extract a sequence $\left(n_{i}\right)_{i \in \mathbb{N}}$ such that $d_{\mathcal{M}}\left(F_{*}^{n_{i}} \mu, \nu\right)<\frac{d}{3}$ and $d_{\mathcal{M}}\left(F_{*}^{n_{i}+1} \mu, \nu\right)>\frac{2 d}{3}$. Since $d_{\mathcal{M}}\left(F_{*}^{n} \mu, \mathcal{V}(F, \mu)\right) \underset{n \rightarrow \infty}{\longrightarrow} 0$ and $\mathbf{B}\left(\nu, \frac{d}{3}\right) \cap \mathcal{V}(F, \mu)=\{\nu\}$, we have $d_{\mathcal{M}}\left(F_{*}^{n_{i}} \mu, \nu\right) \underset{i \rightarrow \infty}{\longrightarrow} 0$. By continuity of $F_{*}$, one has $d_{\mathcal{M}}\left(F_{*}^{n_{i}+1} \mu, F_{*} \nu\right) \underset{i \rightarrow \infty}{\longrightarrow} 0$.

One deduces that for all $\nu \in \mathcal{V}(F, \mu)$ there exists $\nu^{\prime} \in \mathcal{V}(F, \mu)$ such that $F_{*} \nu=\nu^{\prime}$. So there is $k \in \mathbb{N}$ such that $\mathcal{V}(F, \mu)=\left\{\nu_{0}, \ldots, \nu_{k-1}\right\}$ and $F_{*} \nu_{i}=\nu_{i+1}$ where the addition is modulo $k$.

Furthermore, if $\mu$ is computable, then $\mathcal{V}(F, \mu)$ is $\Pi_{2}$-computable and every isolated point is $\Delta_{2}$ computable. In particular if $\mathcal{V}(F, \mu)$ is finite, every point is $\Delta_{2}$-computable. We exhibit some examples of more sophisticated behaviours based on the construction in Theorem 11. The first one is a family of cellular automata where $\mathcal{V}(F, \mu)$ is a finite set of connected components mapped by some periodic CA, which is a partial reciprocal of Proposition 12. The second one is a family of cellular automata where $\mathcal{V}(F, \mu)$ has an infinite number of connected components.

Example 3 (Finite set of connected components). Suppose $\mathcal{K}=\left\{\nu_{0}, \ldots, \nu_{k-1}\right\} \subset \mathcal{M}_{\sigma}\left(\mathcal{B}^{\mathbb{Z}}\right)$ is a finite set of $\sigma$-invariant limit-computable measures such that $G \nu_{i}=\nu_{i+1}$ for some periodic cellular automaton $G: \mathcal{B}^{\mathbb{Z}} \rightarrow \mathcal{B}^{\mathbb{Z}}\left(G^{p}=I d\right.$ for some $\left.p \in \mathbb{N}\right)$. Then there is an alphabet $\mathcal{A} \supset \mathcal{B}$ and a cellular automaton $F: \mathcal{A}^{\mathbb{Z}} \rightarrow \mathcal{A}^{\mathbb{Z}}$ such that $\mathcal{V}(F, \mu)=\mathcal{K}$ for $\mu \in \mathcal{M}_{\sigma-\operatorname{erg}}^{\text {full }}\left(\mathcal{A}^{\mathbb{Z}}\right)$. Indeed, let $F$ be the cellular automaton satisfying $F_{*}^{t} \mu \rightarrow \nu_{0}$ obtained by Theorem 1, and consider the cellular automaton that applies $G$ on the main layer and applies the local rule of $F$ once every $k$ steps. Since $G$ is periodic, the sample produced by the process of copy stay near form $\left\{\nu_{0}, \ldots, \nu_{k-1}\right\}$.

The same idea holds if $\mathcal{K}$ is a finite union of $\Pi_{2}$-CCC sets which are mapped by a periodic cellular automaton $G: \mathcal{B}^{\mathbb{Z}} \rightarrow \mathcal{B}^{\mathbb{Z}}$.

Example 4 (Infinite set of connected components). We give a sketch of a modification of the construction of Theorem 1 to obtain examples of cellular automata where $\mathcal{V}(F, \mu)$ has an infinite number of connected components. This is the first such example to our knowledge. The construction uses the firing squad cellular automaton introduced by Mazoyer [Maz96] $F_{\mathrm{FS}}: \mathcal{B}_{\mathrm{FS}}^{\mathbb{Z}} \rightarrow \mathcal{B}_{\mathrm{FS}}^{\mathbb{Z}}$, which has the following properties:

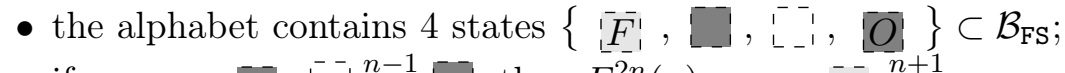

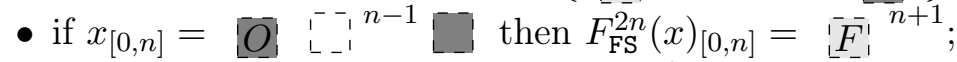

- the state $F_{-}$does not appear in $\left(F_{\mathrm{FS}}^{t}(x)_{j}\right)_{(t, j) \in[0, n] \times[0,2 n-1]}$.

Consider a uniformly computable sequence $\left(\mathcal{K}_{i}\right)_{i \in \mathbb{N}}$ of disjoint $\Pi_{2}$-CCC subsets of $\mathcal{M}_{\sigma}\left(\mathcal{B}^{\mathbb{Z}}\right)$. There is a uniformly computable sequence of words $\left(w_{n}\right)_{n \in \mathbb{N}}$ of $\mathcal{B}^{*}$ such that $\mathcal{V}\left(\left(w_{n}\right)_{n \in \mathbb{N}}=\bigcup_{i \in \mathbb{N}} \mathcal{K}_{i}\right.$. Define $w_{n}^{\prime}=w_{n} \times L_{-1}^{-}\left|w_{n}\right| \in\left(\mathcal{B} \times \mathcal{B}_{\mathrm{FS}}\right)^{*}$ and consider the cellular automaton $F: \mathcal{A}^{\mathbb{Z}} \rightarrow \mathcal{A}^{\mathbb{Z}}$ given by Theorem 1 which produces $\mathcal{V}\left(\left(w_{n}^{\prime}\right)_{n \in \mathbb{N}}\right)$, with $\mathcal{A} \supset \mathcal{B} \times \mathcal{B}_{\mathrm{FS}}$. We modify $F$ to obtain $\widetilde{F}$ in the following way.

- at time $T_{n}$, when the copy of $w_{n}$ is initiated, we initialise a counter on another layer to count the length $k$ of the segment; 
- at time $t=T_{n+1}-2 k$, the state $O$ appears on the left border of each segment (this is a computable number and the time counter keeps track of current time);

- All ${ }_{i} \overline{F_{-}}$symbols are immediately transformed into ${ }^{-}{ }_{-}$symbols.

This requires the segments to be shorter than $T_{n+1}-T_{n}$ cells, but the probability that $[0, l]$ belongs to such a segment tends to 1 as time tends to infinity (Proposition 9). Furthermore, the state $\overline{F^{\prime}}$ appears only at times $\left(T_{n}\right)_{n \in \mathbb{N}}$. Therefore, in those segments, $\widetilde{F_{*}} \mu$ approximates the measure $\widehat{\delta_{w_{n}}} \times \widehat{\delta_{\mathbb{F}_{j}}}$ at time $T_{n+1}$ and the measure $\widehat{\delta_{w_{n}}} \times \widehat{\delta_{\mathrm{L},}}$ at time $T_{n+1}+1$.

For an initial measure $\mu \in \mathcal{M}_{\sigma-\text { mix }}^{\text {full }}\left(\mathcal{A}^{\mathbb{Z}}\right)$, one has $\mathcal{V}(\widetilde{F}, \mu)=\left(\bigcup_{i} \mathcal{K}_{i}\right) \times \widehat{\delta_{\left[F_{i}\right.}} \cup \mathcal{K}^{\prime}$ for some $\mathcal{K}^{\prime} \subset$ $\mathcal{M}_{\sigma}\left(\mathcal{B} \times\left(\mathcal{B}_{\mathrm{FS}} \backslash\left\{\overline{F_{-}}\right\}\right)^{\mathbb{Z}}\right)$. In particular, $\mathcal{V}(\widetilde{F}, \mu)$ has an infinite number of connected components.

Open question 3. Is it possible to characterise all compact subsets of $\mathcal{M}_{\sigma}\left(\mathcal{A}^{\mathbb{Z}}\right)$ that can be reached as $\mu$-limit measures set of some cellular automaton when $\mu$ is computable?

\subsection{Convergence in Cesàro mean}

In this section, by adapting the enumeration $\left(w_{n}\right)_{n \in \mathbb{N}}$, we obtain similar results on $\mathcal{V}^{\prime}(F, \mu)$, the set of limit points for the Cesàro mean sequence. It is easy to prove that $\mathcal{V}^{\prime}(F, \mu)$ is nonempty, connected and included in the convex hull of $\mathcal{V}(F, \mu)$.

Corollary 4. Let $\mathcal{B}$ be a finite alphabet and $\mathcal{K}^{\prime} \subset \mathcal{M}_{\sigma}\left(\mathcal{B}^{\mathbb{Z}}\right)$ a $\Pi_{2}-C C C$ set. There exist an alphabet $\mathcal{A} \supset \mathcal{B}$, and a cellular automaton $F: \mathcal{A}^{\mathbb{Z}} \rightarrow \mathcal{A}^{\mathbb{Z}}$ such that for any $\mu \in \mathcal{M}_{\sigma-\text { mix }}^{\text {full }}\left(\mathcal{A}^{\mathbb{Z}}\right)$, one has $\mathcal{V}^{\prime}(F, \mu)=\mathcal{K}^{\prime}$.

This a full characterisation of sets that can be reached from some initial measure $\mu \in \mathcal{M}_{\sigma}^{\text {comp }}\left(\mathcal{A}^{\mathbb{Z}}\right)$ as $\mu$-limit measures set in Cesàro mean. This corollary is a consequence of the following stronger result, where we have control over both $\mathcal{V}(F, \mu)$ and $\mathcal{V}^{\prime}(F, \mu)$.

Corollary 5. Let $\mathcal{B}$ be a finite alphabet and $\mathcal{K}^{\prime} \subset \mathcal{K} \subset \mathcal{M}_{\sigma}\left(\mathcal{B}^{\mathbb{Z}}\right)$ two $\Pi_{2}-C C C$ sets. There exist an alphabet $\mathcal{A} \supset \mathcal{B}$ and a cellular automaton $F: \mathcal{A}^{\mathbb{Z}} \rightarrow \mathcal{A}^{\mathbb{Z}}$ such that for any $\mu \in \mathcal{M}_{\sigma-\text { mix }}^{\text {full }}\left(\mathcal{A}^{\mathbb{Z}}\right)$, one has:

- $\mathcal{V}(F, \mu)=\mathcal{K}$;

- $\mathcal{V}^{\prime}(F, \mu)=\mathcal{K}^{\prime}$.

This is a full characterisation of pairs of connected subsets $\left(\mathcal{K}, \mathcal{K}^{\prime}\right)$ such that $\mathcal{K}^{\prime} \subset \mathcal{K}$ that can be reached from some initial measure $\mu \in \mathcal{M}_{\sigma}^{\text {comp }}\left(\mathcal{A}^{\mathbb{Z}}\right)$ in this way.

Proof. We use notations from the proof of Proposition [6. Notably $\left(w_{n}\right)_{n \in \mathbb{N}}$ and $\left(w_{n}^{\prime}\right)_{n \in \mathbb{N}}$ are the uniformly computable sequences of words associated to $\mathcal{K}$ and $\mathcal{K}^{\prime}$, respectively, and $\mathbf{V}_{k}$ and $\mathbf{V}_{k}^{t}$ are defined with regard to $\mathcal{K}$.

We define a new sequence of words $\left(w_{n}^{\prime \prime}\right)_{n \in \mathbb{N}}$ in the following manner, using a similar method as Proposition 6. For $n \in \mathbb{N}$, let $i_{n} \leq n$ be the maximal value such that one can find a path $w_{n}=$ $u_{0}, u_{1}, \ldots, u_{l}=w_{n}^{\prime}, u_{l+1}, \ldots, u_{l^{\prime}}=w_{n+1}$ with $u_{1}, \ldots u_{l-1}, u_{l+1}, \ldots, u_{l^{\prime}} \in V_{i_{n}}^{t}$ and $d_{\mathcal{M}}\left(u_{k}, u_{k+1}\right) \leq$ $4 b\left(i_{n}\right)$ for all $k \in\left[0, l^{\prime}\right]$.

Let $P_{n}:\left\{0, \ldots, p_{n}\right\} \rightarrow \mathbf{V}_{i_{n}}^{t}$ be such a path. Since $\mathbf{V}_{i_{n}}^{t} \subset \mathcal{A}^{\leq i_{n}+1}$, this path is of length $p_{n} \leq$ $2|A|^{i_{n}+1} \leq 2|A|^{\left|w_{n}\right|+1}<2|A|^{n+1}$.

For $i \in\left[|\mathcal{A}|^{n^{2}},|\mathcal{A}|^{(n+1)^{2}}\right]$, we define:

- if $i<|\mathcal{A}|^{n^{2}}+p_{n}, w_{i}^{\prime \prime}=P_{n}\left(i-|\mathcal{A}|^{n^{2}}\right)$;

- otherwise, $w_{i}^{\prime \prime}=w_{n}^{\prime}$. 
and let $F$ be the CA associated to $\left(w_{n}^{\prime \prime}\right)_{n \in \mathbb{N}}$ by Theorem 1, Since all elements of $\left(w_{n}\right)_{n \in \mathbb{N}}$ appear, we can prove as in Proposition 6 that $\mathcal{V}(F, \mu)=\mathcal{V}\left(\left(w_{n}^{\prime \prime}\right)_{n \in \mathbb{N}}\right)=\mathcal{K}$.

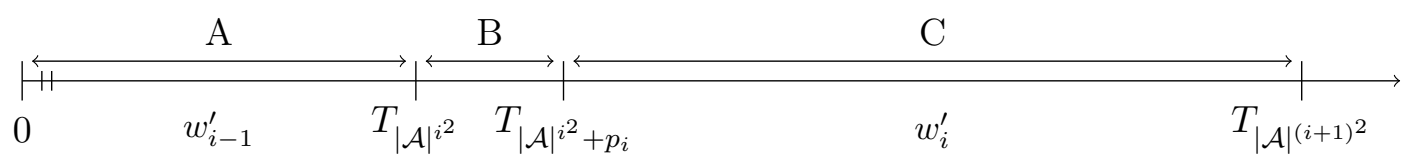

Figure 17. Intuitively, we prove $A+B \ll C$, then $B \ll A$.

We have

$$
\frac{|\mathcal{A}|^{n^{2}}+p_{n}}{|\mathcal{A}|^{(n+1)^{2}}-\left(|\mathcal{A}|^{n^{2}}+p_{n}\right)}<\frac{|\mathcal{A}|^{n^{2}+1}}{|\mathcal{A}|^{(n+1)^{2}}-|\mathcal{A}|^{n^{2}+1}} \underset{n \rightarrow \infty}{\longrightarrow} 0 .
$$

In other words, the subset $\left[0,|\mathcal{A}|^{n^{2}}+p_{n}\right]$ is (asymptotically) of negligible density in $\left[0,|\mathcal{A}|^{(n+1)^{2}}\right]$. Since $T_{i+1}-T_{i}=q^{\lfloor\sqrt{i}\rfloor}$ (where $q$ is defined in Section [3.3.2) is an increasing sequence, the subset $\left[0, T_{|\mathcal{A}|^{n^{2}+p_{n}}}\right]$ is of negligible density in $\left[0, T_{|\mathcal{A}|^{(n+1)^{2}}}\right]$. This means that, putting $t_{n+1}=T_{|\mathcal{A}|^{(n+1)^{2}}}$, $d\left(\varphi_{t_{n+1}}^{F}(\mu), \widehat{\delta_{w_{n+1}^{\prime}}}\right) \underset{n \rightarrow \infty}{\longrightarrow} 0$.

Furthermore, notice that for $x, y \in \mathbb{R}_{+}$, when $y \leq \sqrt{x}$, we have $\lfloor\sqrt{x+y}\rfloor \leq\lfloor\sqrt{x}\rfloor+1$ and $\lfloor\sqrt{x-y}\rfloor \geq\lfloor\sqrt{x}\rfloor-1$. Thus :

and therefore

$$
\begin{gathered}
T_{|\mathcal{A}|^{n^{2}}+p_{n}}-T_{|\mathcal{A}|^{n^{2}}}<q^{|\mathcal{A}|^{\frac{n^{2}}{2}}+1} \cdot 2|\mathcal{A}|^{n+1} \cdot \\
T_{|\mathcal{A}|^{n^{2}+p_{n}}}>T_{|\mathcal{A}|^{n^{2}}}-T_{|\mathcal{A}|^{n^{2}}-|\mathcal{A}|^{\frac{n^{2}}{2}}}>q^{|\mathcal{A}|^{\frac{n^{2}}{2}}}-1 \cdot|\mathcal{A}|^{\frac{n^{2}}{2}}
\end{gathered}
$$

$$
\frac{T_{|\mathcal{A}|^{n^{2}}+p_{n}}-T_{|\mathcal{A}|^{n^{2}}}}{T_{|\mathcal{A}|^{n^{2}}+p_{n}}} \underset{n \rightarrow \infty}{\longrightarrow} 0 .
$$

This means that, putting $t_{n}^{\prime}=T_{|\mathcal{A}|^{n^{2}}+p_{n}}, d\left(\varphi_{t_{n}^{\prime}}^{F}(\mu), \widehat{\delta_{w_{n}^{\prime}}}\right) \underset{n \rightarrow \infty}{\longrightarrow} 0$.

To sum up, we have two sequences of times $t_{0}<t_{0}^{\prime}<\cdots<t_{n}<t_{n}^{\prime}<\ldots$ such that, for all $n \in \mathbb{N}$, the Cesàro mean sequence $\left(\varphi_{t}^{F}(\mu)\right)_{t \in \mathbb{N}}$ is (asymptotically) close to $\widehat{\delta_{w_{n}^{\prime}}}$ between times $t_{n}$ and $t_{n}^{\prime}$, and is close to $\widehat{\delta_{w_{n+1}^{\prime}}}$ at time $t_{n+1}$. Furthermore, between times $t_{n}^{\prime}$ and $t_{n+1}, \varphi_{t}^{F}(\mu)$ is by definition

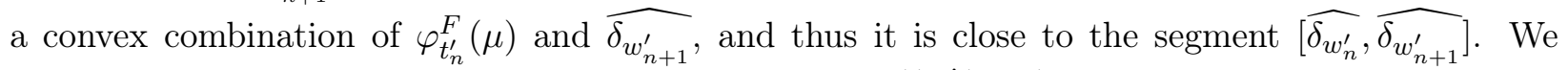
conclude that asymptotically, the sequence is close to $\mathcal{V}\left(\left(w_{n}^{\prime}\right)_{n \in \mathbb{N}}\right)$, and thus its set of limit points is $\mathcal{K}^{\prime}$.

Open question 4. Is it possible to extend Corollary 5 when $\mathcal{K}^{\prime}$ is not included in $\mathcal{K}$ ?

Using Example 3 we can only provide some examples where $\mathcal{V}(F, \mu) \cap \mathcal{V}^{\prime}(F, \mu)=\emptyset$.

This result has a counterpart with no auxiliary states, using Theorem 2 ,

Corollary 6. Let $\mathcal{K}^{\prime} \subset \mathcal{K} \subset \mathcal{M}_{\sigma}\left(\mathcal{B}^{\mathbb{Z}}\right)$ two nonempty $\Pi_{2}$-CCC sets that both do not charge the same word $u \in \mathcal{B}^{*}$. Then there exists a cellular automaton $F: \mathcal{B} \rightarrow \mathcal{B}$ such that for any $\mu \in \mathcal{M}_{\sigma-\text { mix }}^{\text {full }}\left(\mathcal{A}^{\mathbb{Z}}\right)$,

- $\mathcal{V}(F, \mu)=\mathcal{K}$;

- $\mathcal{V}^{\prime}(F, \mu)=\mathcal{K}^{\prime}$. 


\subsection{Undecidability consequences}

We give an undecidability result extending a result of Delacourt on $\mu$-limit sets [Del11].

Corollary 7 (Rice theorem on $\mu$-limit measures sets). Let $P$ be a nontrivial property on non-empty $\Pi_{2}$-CCC sets of $\mathcal{M}_{\sigma}\left(\mathcal{B}^{\mathbb{Z}}\right)$ (i.e. not always or never true). There is no algorithm that can decide, given an alphabet $\mathcal{A}$ and a $C A F: \mathcal{A}^{\mathbb{Z}} \rightarrow \mathcal{A}^{\mathbb{Z}}$, whether $\mathcal{V}(F, \mu)$ satisfies $P$ for $\mu \in \mathcal{M}_{\sigma-\text { mix }}^{\text {full }}\left(\mathcal{B}^{\mathbb{Z}}\right)$.

Proof. We proceed by reduction to the halting problem. Since $P$ is nontrivial, let $\mathcal{K}_{1}$ and $\mathcal{K}_{2}$ be two $\Pi_{2}$-CCC sets that satisfies and does not satisfy $P$, respectively. By Proposition 6 , there exists two uniformly computable sequences of words $\left(w_{n}\right)_{n \in \mathbb{N}},\left(w_{n}^{\prime}\right)_{n \in \mathbb{N}} \in\left(\mathcal{A}^{*}\right)^{\mathbb{N}}$ such that $\mathcal{K}_{1}=$ $\mathcal{V}\left(\left(w_{n}\right)_{n \in \mathbb{N}}\right), \mathcal{K}_{2}=\mathcal{V}\left(\left(w_{n}^{\prime}\right)_{n \in \mathbb{N}}\right)$.

Now let $\mathcal{T} \mathcal{M}$ be a Turing machine. Define the sequence $\left(w_{n}^{\prime \prime}\right)_{n \in \mathbb{N}}$ in the following way.

- If $\mathcal{T} \mathcal{M}$ halts on the empty input in less than $n$ steps, $w_{n}^{\prime \prime}=w_{n}$.

- Otherwise, $w_{n}^{\prime \prime}=w_{n}^{\prime}$.

This sequence is computable by simulating $n$ steps of the Turing machine and outputting the corresponding word. Therefore, we can use the previous construction to build a CA $F$ such that $\mathcal{V}(F, \mu)=\mathcal{V}\left(\left(w_{n}^{\prime \prime}\right)_{n \in \mathbb{N}}\right)$. If $\mathcal{T} \mathcal{M}$ halts on the empty input, then $w_{n}^{\prime \prime}=w_{n}$ for $n$ large enough; otherwise, $w_{n}^{\prime \prime}=w_{n}^{\prime}$ for $n$ large enough. Thus, $\mathcal{V}(F, \mu)$ satisfies $P$ if and only if $\mathcal{T} \mathcal{M}$ halts.

In Corollary 7 the alphabet is considered as an input of the problem. A similar result with a fixed alphabet requires to use the construction of Section 4.

Corollary 8. Let $\mathcal{B}$ be an alphabet, $\mu \in \mathcal{M}_{\sigma-m i x}^{\text {full }}\left(\mathcal{B}^{\mathbb{Z}}\right), u \in \mathcal{B}^{*}$, and $P$ be a nontrivial property on non-empty $\Pi_{2}-C C C$ sets that do not charge $u$. There is no algorithm that can decide, given a $C A$ $F: \mathcal{B}^{\mathbb{Z}} \rightarrow \mathcal{B}^{\mathbb{Z}}$, whether $\mathcal{V}(F, \mu)$ satisfies $P$.

A direct extension would not be possible. If $\lambda$ is the uniform Bernoulli measure, the problem of whether $\mathcal{V}(F, \lambda)$ contains only the uniform Bernoulli measure is equivalent to the surjectivity of $F$, which is decidable AP72. In the non-probabilistic setting, the only decidable property about the asymptotic behaviour of CA is surjectivity [GR10]. However, the question of which nontrivial properties on limit measures and $\mu$-limit measures sets with full support are decidable remains open.

Corollaries 7 and 8 extend naturally for a single limit and the Cesàro mean sequence.

\subsection{Computation on the set of measures}

In this section, we modify the construction to perform computation on the space of probability measures, that is, we want the $\mu$-limit measures set to be a function of the initial measure; this requires to keep some information in the construction. When the initial measure is not computable, we can use this information as a "source" of noncomputability to reach $\mu$-limit measures sets that would be unreachable otherwise.

\subsubsection{Computation with oracle}

It is possible to construct an arithmetical hierarchy for computability of measures. More precisely, a measure is $\Delta_{1}$-computable if it is computable and a measure $\mu$ is $\Delta_{n}$-computable for $n \geq 2$ if there exists a computable function $f: \mathcal{A}^{*} \times \mathbb{N}^{n-1} \rightarrow \mathbb{Q}$ such that

$$
\mu([u])=\lim _{i_{n-1} \rightarrow \infty} \lim _{i_{n-2} \rightarrow \infty} \ldots \lim _{i_{1} \rightarrow \infty} f\left(u, i_{1}, \ldots, i_{n-1}\right) \quad \forall u \in \mathcal{A}^{*} .
$$

Similarly as Proposition 3, if $\mu$ is $\Delta_{n}$-computable and $F_{*}^{t} \mu \underset{t \rightarrow \infty}{\longrightarrow} \nu$ then $\nu$ is $\Delta_{n+1}$-computable. In the same way, one can introduce naturally the notion of $\Pi_{n}$-computable closed set. If $\mu$ is $\Delta_{n}$-computable then $\mathcal{V}(F, \mu)$ is $\Pi_{n+1}$-computable. 
In fact, the obstructions shown in Section 2 can be generalised to obstructions on $\mu \longmapsto \mathcal{V}(F, \mu)$, including cases where the initial measure is not necessarily computable, by considering computability with access to an oracle $\mu \in \mathcal{M}_{\sigma}\left(\mathcal{A}^{\mathbb{Z}}\right)$. In all the following, we fix a subset $\mathcal{M} \subset \mathcal{M}_{\sigma}\left(\mathcal{A}^{\mathbb{Z}}\right)$.

A Turing machine with oracle in $\mathcal{M}$ has the same behaviour as a classical Turing machine, except that an oracle $\mu \in \mathcal{M}$ is fixed prior to computation. The machine can query the oracle at any time during the computation by writing $u \in \mathcal{A}^{*}$ and $n \in \mathbb{N}$ on an special additional oracle tape and entering a special oracle state. At this step, the content of the oracle tape is considered as the oracle input and, after one step, the contents of the oracle tape are replaced by an approximation of $\mu([u])$ up to an error $2^{-n}$ and the computation resumes.

Let $X, Y$ two countable sets. A function $f: \mathcal{M} \times X \rightarrow Y$ is computable with oracles in $\mathcal{M}$ if there exists a Turing machine with oracle in $\mathcal{M}$ which takes as input $x \in X$ and returns $y=f(\mu, x) \in Y$, up to reasonable encoding.

Definition 16. A function $\varphi: \mathcal{M} \longrightarrow \mathcal{M}_{\sigma}\left(\mathcal{B}^{\mathbb{Z}}\right)$ is computable with oracles in $\mathcal{M}$ if there exists a computable function with oracles in $\mathcal{M} f: \mathcal{M} \times \mathbb{N} \longrightarrow \mathcal{B}^{*}$ such that $\left|\varphi(\mu)-\widehat{\delta_{f(\mu, n)}}\right| \leq 2^{-n}$. This is an extension of the previous definition where the image is not countable, hence the abuse of notation.

A sequence of functions $\left(f_{n}: \mathcal{M} \times \mathcal{M}_{\sigma}\left(\mathcal{A}^{\mathbb{Z}}\right) \longrightarrow \mathbb{R}\right)_{n \in \mathbb{N}}$ is a uniformly computable sequence of computable functions with oracles in $\mathcal{M}$ if:

- there exists $a: \mathcal{M} \times \mathbb{N} \times \mathbb{N} \times \mathcal{A}^{*} \longrightarrow \mathbb{Q}$ computable with oracles in $\mathcal{M}$ such that

$$
\left|f_{n}\left(\mu, \widehat{\delta_{w}}\right)-a(\mu, n, m, w)\right| \leq \frac{1}{m} \text { for all } \mu \in \mathcal{M}, w \in \mathcal{A}^{*} \text { and } n, m \in \mathbb{N}
$$

- there exists $b: \mathcal{M} \times \mathbb{N} \longrightarrow \mathbb{Q}$ computable with oracles in $\mathcal{M}$ such that $d_{\mathcal{M}}\left(\nu, \nu^{\prime}\right)<b(\mu, m)$ implies $\left|f_{n}(\mu, \nu)-f_{n}\left(\mu, \nu^{\prime}\right)\right| \leq \frac{1}{m}$ for all $\mu \in \mathcal{M}$ and $n, m \in \mathbb{N}$.

Let $\mathfrak{K}$ be the set of compact subsets of $\mathcal{M}_{\sigma}\left(\mathcal{B}^{\mathbb{Z}}\right)$. Defining the computability of a function $\Psi: \mathcal{M} \longrightarrow \mathfrak{K}$ can be done in various ways, similarly as in Proposition 4 . For example, $\Psi$ is $\Pi_{2}$-computable if the distance function $\mu, \nu \longmapsto d_{\Psi(\mu)}(\nu)$ is $\Sigma_{2}$-computable with oracles in $\mathcal{M}$.

The proofs of Section 2 can be adapted in this framework. For any cellular automaton $F$ on $\mathcal{A}^{\mathbb{Z}}$ :

- $\mu \longmapsto F_{*} \mu$ is computable with oracles in $\mathcal{M}_{\sigma}\left(\mathcal{A}^{\mathbb{Z}}\right)$ (equivalent to Proposition 3);

- $\mu \longmapsto \mathcal{V}(F, \mu)$ and $\mu \longmapsto \mathcal{V}^{\prime}(F, \mu)$ are $\Pi_{2}$-computable with oracles in $\mathcal{M} s\left(\mathcal{A}^{\mathbb{Z}}\right)$ (equivalent to Proposition 5 );

- if $\Psi: \mathcal{M} \longrightarrow \mathfrak{K}$ is a $\Pi_{2}$-computable function with oracles in $\mathcal{M}$ and if every element of $\Psi(\mathcal{M})$ is connected, then there exists a computable function $f: \mathcal{M} \times \mathbb{N} \longrightarrow \mathcal{A}^{*}$ with oracles in $\mathcal{M}$ such that $\Psi(\mu)=\mathcal{V}\left((f(\mu, n))_{n \in \mathbb{N}}\right)$, where $\mathcal{V}\left((f(\mu, n))_{n \in \mathbb{N}}\right)$ is the closure of the limit points of the polygonal path (equivalent to Proposition [6).

\subsubsection{Towards a reciprocal}

In this section, we give a partial reciprocal to these obstructions. To use the initial measure $\mu \in$ $\mathcal{M}_{\sigma}\left(\mathcal{A}^{\mathbb{Z}}\right)$ as an oracle, we need to keep some information from the initial configuration. We adapt the original construction in the following way:

Each segment keeps a sample of the initial configuration, using the frequency of patterns inside this sample as an oracle in the computation. We need to ensure that the frequency of a pattern $u \in \mathcal{A}^{k}$ in this sample is close to $\mu([u])$ with a high probability. For this, we use Theorem III.1.7 of Shi96] applied on a measure $\mu \in \mathcal{M}_{\sigma-\text { mix }}^{\text {full }}\left(\mathcal{A}^{\mathbb{Z}}\right)$ that ensures we have an exponential rate of convergence for every length. Formally, for any $k, m, n \in \mathbb{N}, c>0$ :

$$
\mu\left(\left\{x \in \mathcal{A}^{\mathbb{Z}}: \max _{u \in \mathcal{A}^{k}}\left\{\left|\mu([u])-\operatorname{Freq}\left(u, x_{[0, n]}\right)\right|\right\} \geq \varepsilon\right\}\right) \leq(k+m) \psi(m)^{\frac{n}{k}}\left(\frac{n}{k}+1\right)^{\operatorname{Card}(A)^{k}} 2^{-\frac{n c \varepsilon^{2}}{4 k}} .
$$


However, in our construction, we are unable to keep all information from the initial configuration since the formatting process destroys information in the segment. In all the following, we will only keep information about the density of I symbols, and the reached $\mu$-limit set of measures depends on this parameter only. The same method could be adapted to keep information about longer words, only considering the positions of $\mathrm{I}$ symbols.

Theorem 3. Let $\Psi: \mathcal{M}_{\sigma-m i x}^{\text {full }}\left(\{0,1\}^{\mathbb{Z}}\right) \rightarrow \mathfrak{K}$ be a $\Pi_{2}$-computable function where $\mathfrak{K}$ is the set of compact connected subsets of $\mathcal{M}_{\sigma}\left(\mathcal{B}^{\mathbb{Z}}\right)$. Assume that if $\mu, \mu^{\prime} \in \mathcal{M}_{\sigma-\text { mix }}^{\text {full }}\left(\{0,1\}^{\mathbb{Z}}\right)$ are such that $\mu([1])=\mu^{\prime}([1])$, we have $\Psi(\mu)=\Psi\left(\mu^{\prime}\right)$.

Then there exists an alphabet $\mathcal{A} \supset \mathcal{B}$ and a cellular automaton $F: \mathcal{A}^{\mathbb{Z}} \rightarrow \mathcal{A}^{\mathbb{Z}}$ such that for all $\mu \in \mathcal{M}_{\sigma-\text { mix }}^{\text {full }}\left(\mathcal{A}^{\mathbb{Z}}\right)$, we have $\mathcal{V}(F, \mu)=\Psi(\pi \mu)$ where $\pi$ is the 1-block map defined by $\pi(x)_{i}=1$ when $x_{i}=I$, and $\pi(x)_{i}=0$ otherwise.

Notice that since only one density is considered, it would be equivalent in this case to consider a $\Pi_{2}$-computable function with oracles in $\mathbb{R}$ and define a function $\mathbb{R} \rightarrow \mathfrak{K}$. We kept the statement more technical to be consistent with the general case.

Proof. Let $f: \mathcal{M}_{\sigma-\text { mix }}^{\text {full }}\left(\{0,1\}^{\mathbb{Z}}\right) \times \mathbb{N} \longrightarrow \mathcal{A}^{*}$ be a computable function with oracles in $\mathcal{M}_{\sigma-\text { mix }}^{\text {full }}\left(\{0,1\}^{\mathbb{Z}}\right)$ such that $\Psi(\mu)=\mathcal{V}\left((f(\mu, n))_{n \in \mathbb{N}}\right)$ and consider the associated Turing machine with oracle.

Let $F$ be the cellular automaton defined in Theorem 1 that simulated the Turing machine corresponding to $\left((f(\mu, n))_{n \in \mathbb{N}}\right)$. Of course we need to specify the behaviour of the automata when the machine performs an oracle query.

We add a new layer $\mathcal{A}_{\text {oracle }}$ in which each segment at time $t$ stores the frequency of the state $\mathrm{I}$ in this segment at time 0 . To do that, we modify the construction in the following way:

- We subdivide the layer $\mathcal{A}_{\text {oracle }}$ in two parts, on which each wall $\mathrm{W}$ keeps on its left:

- the first counter for the number of I symbols that have been destroyed in its left segment;

- the second counter for the length of this segment, 0 if the segment is not formatted.

- Another counter accompanies each formatting counter, measuring the length of the segment as it progresses.

- The second counter is initialised as 0 . When the time counter attached to this wall makes a comparison with an initialised formatting counter (the comparison returns the result "="), the second counter stores the length of the segment. It may take the value 0 again if it merges with a non-formatted segment (see Figure 18).

- When a wall is destroyed by a merging process, it sends to its right an oracle signal at speed 1 containing the information stored in its oracle counters. Such a signal should not cross a formatting counter, so it is slowed down if necessary.

- When a wall's counters are $\left(c_{1}, c_{2}\right)$ and a signal $\left(c_{1}^{\prime}, c_{2}^{\prime}\right)$ comes from its left, there are three cases:

- If $c_{2}=0$, the left segment cannot be formatted; the signal cannot come from an initialised wall and can be safely ignored. The counters does not change.

- If $c_{2} \neq 0$, the left segment has been formatted and all false signals erased. Thus the information comes from an initialised wall. The new number of I symbols is $c_{1}^{\prime \prime}=c_{1}+c_{1}^{\prime}+1$ to take the merging into account.

$*$ If $c_{2}^{\prime}=0$, the segment just merged with a non-formatted segment and $c_{2}^{\prime \prime}=0$;

$*$ otherwise $c_{2}^{\prime \prime}=c_{2}+c_{2}^{\prime}$.

The counters take the values $\left(c_{1}^{\prime \prime}, c_{2}^{\prime \prime}\right)$.

See Figure 18. We remark that if the length of the segment is $k$, the information can be coded in space $\log (k)$, and it is possible to actualise the values before another signal can come from the left. 


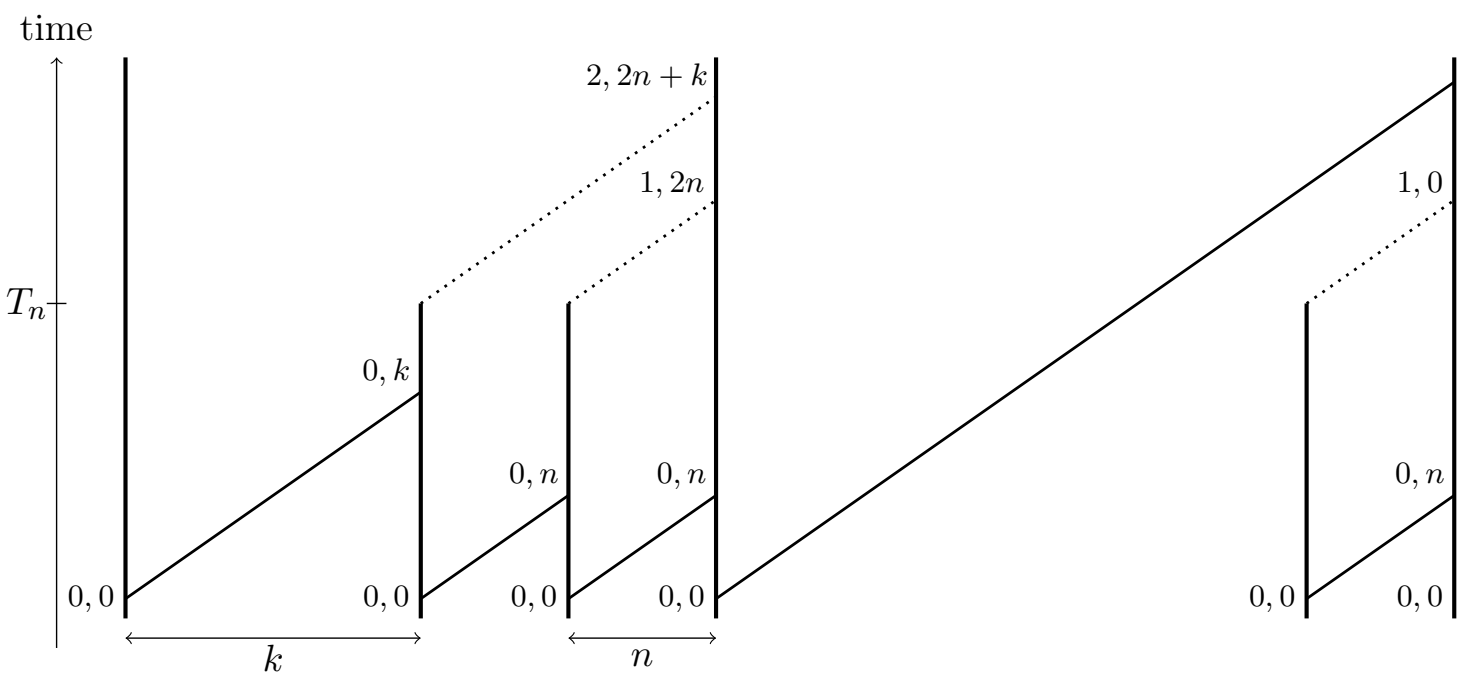

Figure 18. Each wall has its counter displayed when its value changes. Slanted thick lines are formatting counters, dotted lines are signals transmitting information.

- If two symbols I are too close in the initial configuration, they are destroyed by the bootstrapping process (see Section 3.2.1). If a $\mathrm{I}$ is in a group of $\mathrm{I}$ separated by two cells or less, the rightmost $\mathrm{I}$ sends a formatting counter and the leftmost one starts a time counter. Thus a group of $\mathrm{I}$ separated by two cells or less behave as a single symbol for initialisation purposes. Each $\mathrm{I}$ symbol except the leftmost one is transformed immediately into an oracle signal $(1, d)$, where $d$ is the distance to the nearest $\mathrm{I}$ to its left. The other cells present initially are erased.

- The Turing machine simulation described in Section 3.3.2 can be adapted to simulate a Turing machine with oracle. When there is an oracle query for the value of $\mu([\mathrm{I}]])$ with precision $2^{-i}$ at time $t \in\left[T_{n}, T_{n+1}\right]$, there are two possibilities:

- if $n^{-\frac{1}{6}} \leq 2^{-i}$, the Turing machine uses the information stored in the oracle layer to return the frequency of $\mathrm{I}$ on the segment at time 0 , and this corresponds to an approximation of $\mu([[\mathrm{I}]])$ with sufficient precision;

- if $n^{-\frac{1}{6}}>2^{-i}$, the computation stops, and the last word successfully computed is output. The same thing happens until a time when enough information is available.

Let us check that $\mathcal{V}(F, \mu)=\Psi\left(\pi_{*} \mu\right)$ for $\mu \in \mathcal{M}_{\sigma-\text { mix }}^{\text {full }}\left(\mathcal{A}^{\mathbb{Z}}\right)$. It is clear that the density of auxiliary states tends to 0 , so if the sample approximates correctly $\mu([\mathrm{I}])$, the sequence of words $\left(w_{n}\right)_{n \in \mathbb{N}}$ produced by the cellular automaton correspond to $(f(\mu, n))_{n \in \mathbb{N}}$ up to some repetition. Thus we only need to prove that the probability that a cell belongs to a segment whose sample corresponds to a "bad" approximation tends to 0 when $t$ tends to $\infty$. Recall that $\Gamma_{[i, j]}^{T_{n}}=\left\{x \in \mathcal{A}^{\mathbb{Z}} \mid[i, j]\right.$ is a segment at time $\left.T_{n}\right\}$.

$$
\begin{aligned}
B_{n} & =\quad \mu\left(\left\{x \in \mathcal{A}^{\mathbb{Z}}: x_{0} \text { belongs in a segment with a "bad" sample at time } T_{n}\right\}\right) \\
& =\quad \sum_{i<0, j>0} \mu\left(\left\{x \in \Gamma_{[i, j]}^{T_{n}}: \mid \mu([[\mathrm{I}]])-\operatorname{Freq}\left(\left[\mathrm{I}, x_{[i, j]}\right) \mid>n^{-\frac{1}{6}}\right\}\right)\right. \\
& =\quad \sum_{k>0} k \cdot \mu\left(\left\{x \in \Gamma_{[0, k]}^{T_{n}}: \mid \mu([[\mathrm{I}]])-\operatorname{Freq}\left(\left[\mathrm{I}, x_{[0, k]}\right) \mid>n^{-\frac{1}{6}}\right\}\right),\right.
\end{aligned}
$$


by $\sigma$-invariance. By restricting ourselves to $n \leq k \leq K_{n}$, and for any $m \in \mathbb{N}$ large enough that $\psi_{\mu}(m)<1$ :

$$
\begin{array}{rlrl}
B_{n} & \leq & \mu\left(\Gamma_{0, \geq K_{n}}^{T_{n}}\right)+\sum_{k=n}^{K_{n}} k \cdot \mu\left(\left\{x \in \mathcal{A}^{\mathbb{Z}}: \mid \mu\left([[\mathrm{I}])-\operatorname{Freq}\left([\mathrm{I}], x_{[0, k]}\right) \mid>n^{-\frac{1}{6}}\right\}\right)\right. \\
& \leq \quad \mu\left(\Gamma_{0, \geq K_{n}}^{T_{n}}\right)+K_{n}^{2}(1+m) \psi_{\mu}(m)^{n}(n+1)^{\operatorname{Card}(A)} 2^{-\frac{c}{4} n^{\frac{2}{3}}} \\
\underset{n \rightarrow \infty}{\longrightarrow} & 0 .
\end{array}
$$

The result follows.

This result may seem surprising since the same cellular automaton has very different asymptotic behaviours depending on the initial measure.

Open question 5. Can Theorem 3 be extended to characterise functions $\Psi: \mathcal{M}_{\sigma-\text { mix }}^{\text {full }}\left(\{0,1\}^{\mathbb{Z}}\right) \rightarrow \mathfrak{K}$ that are realisable as the action of a cellular automaton $F$ in the sense that for all $\mu, \mathcal{V}(F, \mu)=\Psi(\mu)$ ?

\section{Acknowledgements}

The authors want to thank the anonymous referee for his rigorous and detailed review which helped us to clarify the paper. Moreover, this work was partially supported by the ANR project QuasiCool (ANR-12-JS02-011-01) and the ANR project Valet (ANR-13-JS01-0010).

\section{REFERENCES}

[AP72] S. Amoroso and Y.N. Patt. Decision procedures for surjectivity and injectivity of parallel maps for tessellation structures. Journal of Computer and System Sciences, 6(5):448-464, 1972.

[AS11] Nathalie Aubrun and Mathieu Sablik. Simulation of effective subshifts by two-dimensional subshift of finite type. Acta Applicandae Mathematicae, Volume 128, issue 1, p.35-63, 2013.

[BDS10] Laurent Boyer, Martin Delacourt, and Mathieu Sablik. Construction of $\mu$-limit sets. In $J A C$, pages 76-87, 2010.

[BP11] Mike Boyle and Karl Petersen. Hidden Markov processes in the context of symbolic dynamics. In Entropy of hidden Markov processes and connections to dynamical systems, volume 385 of London Math. Soc. Lecture Note Ser., pages 5-71. Cambridge Univ. Press, Cambridge, 2011.

[BPT06] Laurent Boyer, Victor Poupet, and Guillaume Theyssier. On the complexity of limit sets of cellular automata associated with probability measures. In MFCS, pages 190-201, 2006.

[Del11] Martin Delacourt. Rice's Theorem for $\mu$-Limit Sets of Cellular Automata. In ICALP (2), pages 89-100, 2011.

[dMS11] Benjamin Hellouin de Menibus and Mathieu Sablik. Self-organisation in Cellular Automata: A ParticleBased Approach. In Developments in Language Theory, pages 251-263, 2011.

[dM14] Benjamin Hellouin de Menibus. Asymptotic behaviour of cellular automata: computation and randomness. Ph.D thesis of the University of Aix-Marseille, 2014.

[DPST11] Martin Delacourt, Victor Poupet, Mathieu Sablik, and Guillaume Theyssier. Directional dynamics along arbitrary curves in cellular automata. Theor. Comput. Sci., 412(30):3800-3821, 2011.

[Fis90] Robert Fisch. The one-dimensional cyclic cellular automaton: a system with deterministic dynamics that emulates an interacting particle system with stochastic dynamics. J. Theoret. Probab., 3(2):311-338, 1990.

[FM10] Sébastien Ferenczi and Thierry Monteil. Infinite words with uniform frequencies, and invariant measures. In Valérie Berthé and Michel Rigo, editors, Combinatorics, Automata and Number Theory, pages 374-415. Cambridge University Press, 2010.

[FMMn00] Pablo A. Ferrari, Alejandro Maass, Servet Martínez, and Peter Ney. Cesàro mean distribution of group automata starting from measures with summable decay. Ergodic Theory Dynam. Systems, 20(6):16571670, 2000.

[Gác01] Peter Gács. Reliable cellular automata with self-organisation. J. Statist. Phys., 103(1-2):45-267, 2001. 
[GHR11] Stefano Galatolo, Mathieu Hoyrup et Cristóbal Rojas. Dynamics and abstract computability: computing invariant measures. Discrete and Continuous Dynamical Systems - Series A, 29(1):193-212, janvier 2011.

[GR10] Pierre Guillon and Gaétan Richard. Revisiting the rice theorem of cellular automata. In 27th International Symposium on Theoretical Aspects of Computer Science-STACS 2010, pages 441-452, 2010.

[Hed69] Gustav A. Hedlund. Endomorphisms and automorphisms of the shift dynamical system. Mathematical Systems Theory, 3(4):320-375, 1969.

[HM10] Michael Hochman and Tom Meyerovitch. A characterisation of the entropies of multidimensional shifts of finite type. Ann. of Math. (2), 171(3):2011-2038, 2010.

[Hoc09] Michael Hochman. On the dynamics and recursive properties of multidimensional symbolic systems. Invent. Math., 176(1):131-167, 2009.

[KM00] Petr Kůrka and Alejandro Maass. Limit sets of cellular automata associated to probability measures. J. Statist. Phys., 100(5-6):1031-1047, 2000.

[Ku05] Petr Kůrka. On the measure attractor of a cellular automaton. Discrete Contin. Dyn. Syst., (suppl.):524$535,2005$.

[Lin84] D. A. Lind. Applications of ergodic theory and sofic systems to cellular automata. Physica D: Nonlinear Phenomena, 10(1-2):36-44, 1984.

[Maz96] Jacques Mazoyer. On optimal solutions to the firing squad synchronisation problem. Theoret. Comput. Sci., 168(2):367-404, 1996. Universal machines and computations (Paris, 1995).

[Mey11] Tom Meyerovitch. Growth-type invariants for $\mathbb{Z}^{d}$ subshifts of finite type and arithmetical classes of real numbers. Inventiones Mathematicae, 184:567-589, 2011.

[MM98] Alejandro Maass and Servet Martínez. On Cesàro limit distribution of a class of permutative cellular automata. J. Statist. Phys., 90(1-2):435-452, 1998.

[Pet83] Karl Petersen. Ergodic theory, volume 2 of Cambridge Studies in Advanced Mathematics. Cambridge University Press, Cambridge, 1983.

[PY02] Marcus Pivato and Reem Yassawi. Limit measures for affine cellular automata. Ergodic Theory Dynam. Systems, 22(4):1269-1287, 2002.

[Shi96] Paul C. Shields. The ergodic theory of discrete sample paths, volume 13 of Graduate Studies in Mathematics. American Mathematical Society, Providence, RI, 1996.

[Wei00] Klaus Weihrauch. Computable analysis. Texts in Theoretical Computer Science. An EATCS Series. Springer-Verlag, Berlin, 2000. An introduction.

[You02] Lai-Sang Young. What are SRB measures, and which dynamical systems have them? J. Statist. Phys., 108(5-6):733-754, 2002. Dedicated to David Ruelle and Yasha Sinai on the occasion of their 65th birthdays.

[Zie05] Martin Ziegler. Computability and Continuity on the Real Arithmetic Hierarchy and the Power of Type-2 Nondeterminism. In CiE, pages 562-571, 2005.

[ZW01] Xizhong Zheng and Klaus Weihrauch. The arithmetical hierarchy of real numbers. MLQ Math. Log. Q., 47(1):51-65, 2001.

Aix Marseille Université, CNRS, Centrale Marseille, I2M UMR 7373, 13453, Marseille, France E-mail address: benjamin.hellouin@gmail.com

URL: http://mat-unab.cl/ hellouin/

Aix Marseille Université, CNRS, Centrale Marseille, I2M UMR 7373, 13453, Marseille, France

E-mail address: mathieu.sablik@univ-amu.fr

URL: http://www.i2m.univ-amu.fr/ sablik/ 